\title{
Global Classical and Weak Solutions to the Three-Dimensional Full Compressible Navier-Stokes System with Vacuum and Large Oscillations*
}

\author{
Xiangdi $\mathrm{HUANG}^{a}$, Jing $\mathrm{LI}^{b}$ \\ ${ }^{a}$ Department of Mathematics, \\ University of Science and Technology of China, Hefei 230026, P. R. China \\ ${ }^{b}$ Institute of Applied Mathematics, AMSS, \\ \& Hua Loo-Keng Key Laboratory of Mathematics, \\ Chinese Academy of Sciences, Beijing 100190, P. R. China
}

\begin{abstract}
We establish the global existence and uniqueness of classical solutions to the three-dimensional full compressible Navier-Stokes system with smooth initial data which are of small energy but possibly large oscillations where the initial density is allowed to vanish. Moreover, for the initial data, which may be discontinuous and contain vacuum states, we also obtain the global existence of weak solutions. These results generalize previous ones on classical and weak solutions for initial density being strictly away from vacuum, and are the first for global classical and weak solutions which may have large oscillations and can contain vacuum states.
\end{abstract}

\section{Introduction}

The motion of compressible viscous, heat-conductive, Newtonian polytropic fluid occupying a spatial domain $\Omega \subset \mathbb{R}^{3}$ is governed by the following full compressible Navier-Stokes system:

$$
\left\{\begin{array}{l}
\rho_{t}+\operatorname{div}(\rho u)=0, \\
(\rho u)_{t}+\operatorname{div}(\rho u \otimes u)-\mu \Delta u-(\mu+\lambda) \nabla(\operatorname{div} u)+\nabla P=0, \\
(\rho E)_{t}+\operatorname{div}(\rho E u+P u)=\Delta\left(\kappa \theta+\frac{1}{2} \mu|u|^{2}\right)+\mu \operatorname{div}(u \cdot \nabla u)+\lambda \operatorname{div}(u \operatorname{div} u) .
\end{array}\right.
$$

Here $t \geq 0$ is time, $x \in \Omega$ is the spatial coordinate, and $\rho, u=\left(u^{1}, u^{2}, u^{3}\right)^{\operatorname{tr}}, e, P(\rho, e)$, and $\theta$ represent respectively the fluid density, velocity, specific internal energy, pressure, and absolute temperature, and $E=e+\frac{1}{2}|u|^{2}$ is the specific total energy. The constant viscosity coefficients $\mu$ and $\lambda$ satisfy the physical restrictions:

$$
\mu>0, \quad 2 \mu+3 \lambda \geq 0 .
$$

The equations (1.1) then express respectively the conservation of mass, the balance of momentum, and the balance of energy under internal pressure, viscosity forces, and the

${ }^{*}$ This research is partially supported by SRF for ROCS, SEM, and National Science Foundation of China under grant 10971215. Email: xdhuang@ustc.edu.cn (X. Huang), ajingli@gmail.com (J. Li). 
conduction of thermal energy. We study the ideal polytropic fluids so that $P$ and $e$ are given by the state equations:

$$
P(\rho, e)=(\gamma-1) \rho e=R \rho \theta, \quad e=\frac{R \theta}{\gamma-1},
$$

where $\gamma>1$ is the adiabatic constant, and $R, \kappa$ are both positive constants.

Let $\Omega=\mathbb{R}^{3}$ and $\tilde{\rho}, \tilde{\theta}$ both be fixed positive constants. We look for the solutions, $(\rho(x, t), u(x, t), \theta(x, t))$, to the Cauchy problem for (1.1) with the far field behavior:

$$
(\rho, u, \theta)(x, t) \rightarrow(\tilde{\rho}, 0, \tilde{\theta}), \quad \text { as }|x| \rightarrow \infty, t>0,
$$

and initial data:

$$
(\rho, \rho u, \rho \theta)(x, t=0)=\left(\rho_{0}, \rho_{0} u_{0}, \rho_{0} \theta_{0}\right)(x), \quad x \in \mathbb{R}^{3},
$$

with $\rho_{0} \geq 0, \theta_{0} \geq 0$. Note here that for classical solutions, (1.1) can be rewritten as

$$
\left\{\begin{array}{l}
\rho_{t}+\operatorname{div}(\rho u)=0, \\
\rho\left(u_{t}+u \cdot \nabla u\right)=\mu \Delta u+(\mu+\lambda) \nabla(\operatorname{div} u)-\nabla P, \\
\frac{R}{\gamma-1} \rho\left(\theta_{t}+u \cdot \nabla \theta\right)=\kappa \Delta \theta-P \operatorname{div} u+\lambda(\operatorname{div} u)^{2}+2 \mu|\mathfrak{D}(u)|^{2},
\end{array}\right.
$$

where $\mathfrak{D}(u)$ is the deformation tensor:

$$
\mathfrak{D}(u)=\frac{1}{2}\left(\nabla u+(\nabla u)^{\operatorname{tr}}\right)
$$

Moreover, for classical solutions, we replace the initial condition (1.5) with

$$
(\rho, u, \theta)(x, t=0)=\left(\rho_{0}, u_{0}, \theta_{0}\right), \quad x \in \mathbb{R}^{3} .
$$

There is a lot of literature on the large time existence and behavior of solutions to (1.1). The one-dimensional problem with strictly positive initial density and temperature has been studied extensively by many people, see [1, 14, 15] and the references therein. For the multi-dimensional case, the local existence and uniqueness of classical solutions are known in [19,22] in the absence of vacuum. Recently, for the case that the initial density need not be positive and may vanish in open sets, Cho-Kim [6] obtained the local existence and uniqueness of strong solutions. The global classical solutions were first obtained by Matsumura-Nishida [18] for initial data close to a nonvacuum equilibrium in some Sobolev space $H^{s}$. In particular, the theory requires that the solution has small oscillations from a uniform non-vacuum state so that the density is strictly away from vacuum and the gradient of the density remains bounded uniformly in time. Later, Hoff [10] studied the global weak solutions with strictly positive initial density and temperature for discontinuous initial data. On the other hand, in the presence of vacuum, this issue becomes much more complicated. Concerning viscous compressible fluids in a barotropic regime, where the state of these fluids at each instant $t>0$ is completely determined by the density $\rho=\rho(x, t)$ and the velocity $u=u(x, t)$, the pressure $P$ being an explicit function of the density, the major breakthrough is due to Lions [17] (see also Feireisl [7, 9]), where he obtained global existence of weak solutions, defined as solutions with finite energy, when the pressure $P(\rho)=a \rho^{\gamma}(a>0, \gamma>1)$ with suitably large $\gamma$. The main restriction on initial data is that the initial energy is finite, so that the density vanishes at far fields, or even 
has compact support. Recently, Huang-Li-Xin [13] established the global existence and uniqueness of classical solutions to the Cauchy problem for the isentropic compressible Navier-Stokes equations in three-dimensional space with smooth initial data which are of small energy but possibly large oscillations; in particular, the initial density is allowed to vanish, even has compact support. This result can be regarded as uniqueness and regularity theory of Lions-Feireisl's weak solutions in [7, 9, 17] with small initial energy.

However, the global well-posedness of classical solutions, even the global existence of weak solutions to (1.1) remains completely open in the presence of vacuum. For specific pressure laws excluding the perfect gas equation of state, the question of existence of socalled "variational" solutions in dimension $d \geq 2$ has been recently addressed in [7, 8], where the temperature equation is satisfied only as an inequality which justifies the notion of variational solutions. Let us emphasize that this work is the very first attempt towards the existence of weak solutions to the full compressible Navier-Stokes system for large initial data with vacuum. Recently, for a very particular form of the viscosity coefficients depending on the density, Bresch-Desjardins [3] obtained global stability of weak solutions. It is worth noting here that Xin [23] first established remarkable blowup results, which show that in the case that the initial density has compact support, any smooth solution to the Cauchy problem of the full compressible Navier-Stokes system without heat conduction blows up in finite time. See also the recent generalizations to the cases for the full compressible Navier-Stokes system with heat conduction ( 4]) and for non-compact but rapidly decreasing at far field initial densities ( [21]).

Motivated by our previous work on the isentropic compressible Navier-Stokes equations [13], we try to look for the global existence of classical and weak solutions to the three-dimensional full compressible Navier-Stokes system (1.1); in particular, the initial density is allowed to vanish.

Before stating the main results, we explain the notations and conventions used throughout this paper. We denote

$$
\int f d x=\int_{\mathbb{R}^{3}} f d x
$$

For $1 \leq p \leq \infty$ and integer $k \geq 0$, we adopt the simplified notations for the standard homogeneous and inhomogeneous Sobolev spaces as follows:

$$
\left\{\begin{array}{l}
L^{p}=L^{p}\left(\mathbb{R}^{3}\right), \quad W^{k, p}=W^{k, p}\left(\mathbb{R}^{3}\right), \quad H^{k}=W^{k, 2}, \\
D^{1}=\left\{u \in L^{6} \mid\|\nabla u\|_{L^{2}}<\infty\right\}, \quad D^{1, p}=\left\{u \in L_{l o c}^{1}\left(\mathbb{R}^{3}\right) \mid\|\nabla u\|_{L^{p}}<\infty\right\} .
\end{array}\right.
$$

Without loss of generality, we assume that $\tilde{\rho}=\tilde{\theta}=1$. We define the initial energy $C_{0}$ as follows:

$$
\begin{aligned}
C_{0} \triangleq & \frac{1}{2} \int \rho_{0}\left|u_{0}\right|^{2} d x+R \int\left(\rho_{0} \log \rho_{0}-\rho_{0}+1\right) d x \\
& +\frac{R}{\gamma-1} \int \rho_{0}\left(\theta_{0}-\log \theta_{0}-1\right) d x+\frac{R}{2(\gamma-1)} \int \rho_{0}\left(\theta_{0}-1\right)^{2} d x .
\end{aligned}
$$

Then the first main result in this paper can be stated as follows:

Theorem 1.1 For given numbers $M>0$ (not necessarily small), $q \in(3,6)$, and $\bar{\rho}>2$, suppose that the initial data $\left(\rho_{0}, u_{0}, \theta_{0}\right)$ satisfies

$$
\rho_{0}-1 \in H^{2} \cap W^{2, q}, \quad u_{0} \in H^{2}, \quad \theta_{0}-1 \in H^{2},
$$




$$
0 \leq \inf \rho_{0} \leq \sup \rho_{0}<\bar{\rho}, \quad \inf \theta_{0} \geq 0, \quad\left\|\nabla u_{0}\right\|_{L^{2}} \leq M,
$$

and the compatibility conditions:

$$
\begin{aligned}
-\mu \Delta u_{0}-(\mu+\lambda) \nabla \operatorname{div} u_{0}+R \nabla\left(\rho_{0} \theta_{0}\right) & =\sqrt{\rho_{0}} g_{1}, \\
\kappa \Delta \theta_{0}+\frac{\mu}{2}\left|\nabla u_{0}+\left(\nabla u_{0}\right)^{\operatorname{tr}}\right|^{2}+\lambda\left(\operatorname{div} u_{0}\right)^{2} & =\sqrt{\rho_{0}} g_{2},
\end{aligned}
$$

with $g_{1}, g_{2} \in L^{2}$. Then there exists a positive constant $\varepsilon$ depending only on $\mu, \lambda, \kappa, R$, $\gamma, \bar{\rho}$, and $M$ such that if

$$
C_{0} \leq \varepsilon
$$

the Cauchy problem (1.6) (1.4) (1.7) has a unique global classical solution $(\rho, u, \theta)$ in $\mathbb{R}^{3} \times(0, \infty)$ satisfying

$$
\begin{gathered}
0 \leq \rho(x, t) \leq 2 \bar{\rho}, \quad \theta(x, t) \geq 0, \quad x \in \mathbb{R}^{3}, t \geq 0, \\
\left\{\begin{array}{l}
\rho-1 \in C\left([0, T] ; H^{2} \cap W^{2, q}\right), \quad(u, \theta-1) \in C\left([0, T] ; H^{2}\right), \\
u \in L^{\infty}\left(\tau, T ; H^{3} \cap W^{3, q}\right), \quad \theta-1 \in L^{\infty}\left(\tau, T ; H^{4}\right), \\
\left(u_{t}, \theta_{t}\right) \in L^{\infty}\left(\tau, T ; H^{2}\right) \cap H^{1}\left(\tau, T ; H^{1}\right),
\end{array}\right.
\end{gathered}
$$

and the following large-time behavior:

$$
\lim _{t \rightarrow \infty}\left(\|\rho(\cdot, t)-1\|_{L^{p}}+\|\nabla u(\cdot, t)\|_{L^{r}}+\|\nabla \theta(\cdot, t)\|_{L^{r}}\right)=0
$$

with any

$$
0<\tau<T<\infty, \quad p \in(2, \infty), \quad r \in[2,6) .
$$

The next result of this paper will treat the weak solutions due to the fact that discontinuous solutions are fundamental both in the physical theory of nonequilibrium thermodynamics as well as in the mathematical theory of inviscid models for compressible fluids. To begin with, we give the definition of weak solutions.

Definition 1.1 We say that $\left(\rho, u, E=\frac{1}{2}|u|^{2}+\frac{R}{\gamma-1} \theta\right)$ is a weak solution to Cauchy problem (1.1) (1.4) (1.5) provided that

$$
\rho-1 \in L_{\mathrm{loc}}^{\infty}\left([0, \infty) ; L^{2} \cap L^{\infty}\left(\mathbb{R}^{3}\right)\right), \quad u, \theta-1 \in L^{2}\left(0, \infty ; H^{1}\left(\mathbb{R}^{3}\right)\right),
$$

and that for all test functions $\psi \in \mathcal{D}\left(\mathbb{R}^{3} \times(-\infty, \infty)\right)$,

$$
\begin{gathered}
\int_{\mathbb{R}^{3}} \rho_{0} \psi(\cdot, 0) d x+\int_{0}^{\infty} \int_{\mathbb{R}^{3}}\left(\rho \psi_{t}+\rho u \cdot \nabla \psi\right) d x d t=0, \\
\int_{\mathbb{R}^{3}} \rho_{0} u_{0}^{j} \psi(\cdot, 0) d x+\int_{0}^{\infty} \int_{\mathbb{R}^{3}}\left(\rho u^{j} \psi_{t}+\rho u^{j} u \cdot \nabla \psi+P(\rho, \theta) \psi_{x_{j}}\right) d x d t \\
-\int_{0}^{\infty} \int_{\mathbb{R}^{3}}\left(\mu \nabla u^{j} \cdot \nabla \psi+(\mu+\lambda)(\operatorname{div} u) \psi_{x_{j}}\right) d x d t=0, \quad j=1,2,3, \\
\int_{\mathbb{R}^{3}}\left(\frac{1}{2} \rho_{0}\left|u_{0}\right|^{2}+\frac{R}{\gamma-1} \rho_{0} \theta_{0}\right) \psi(\cdot, 0) d x \\
=\int_{0}^{\infty} \int_{\mathbb{R}^{3}}\left(\rho E \psi_{t}+(\rho E+P) u \cdot \nabla \psi\right) d x d t \\
-\int_{0}^{\infty} \int_{\mathbb{R}^{3}}\left(\kappa \nabla \theta+\frac{1}{2} \mu \nabla\left(|u|^{2}\right)+\mu u \cdot \nabla u+\lambda u \operatorname{div} u\right) \cdot \nabla \psi d x d t .
\end{gathered}
$$


We also define

$$
\dot{f} \triangleq f_{t}+u \cdot \nabla f, \quad G \triangleq(2 \mu+\lambda) \operatorname{div} u-R(\rho \theta-1), \quad \omega \triangleq \nabla \times u,
$$

which are the material derivative of $f$, the effective viscous flux, and the vorticity respectively. We now state our second main result as follows:

Theorem 1.2 For given numbers $M>0$ (not necessarily small), and $\bar{\rho}>2$, there exists a positive constant $\varepsilon$ depending only on $\mu, \lambda, \kappa, R, \gamma, \bar{\rho}$, and $M$ such that if the initial data $\left(\rho_{0}, u_{0}, \theta_{0}\right)$ satisfies (1.10) and

$$
C_{0} \leq \varepsilon
$$

with $C_{0}$ as in (1.8), there is a global weak solution $\left(\rho, u, E=\frac{1}{2}|u|^{2}+\frac{R}{\gamma-1} \theta\right)$ to the Cauchy problem (1.1) 1.4) (1.5) satisfying

$$
\begin{gathered}
\rho-1 \in C\left([0, \infty) ; L^{2} \cap L^{p}\right), \quad\left(\rho u, \rho|u|^{2}, \rho(\theta-1)\right) \in C\left([0, \infty) ; H^{-1}\right), \\
u \in C\left((0, \infty) ; L^{2}\right), \quad \theta-1 \in C\left((0, \infty) ; W^{1, r}\right), \\
u(\cdot, t), \omega(\cdot, t), G(\cdot, t), \quad \nabla \theta(\cdot, t) \in H^{1}, \quad t>0, \\
\rho \in[0,2 \bar{\rho}] \quad \text { a.e., } \quad \theta \geq 0 \quad \text { a.e. },
\end{gathered}
$$

and the following large-time behavior:

$$
\lim _{t \rightarrow \infty}\left(\|\rho(\cdot, t)-1\|_{L^{p}}+\|u(\cdot, t)\|_{L^{p} \cap L^{\infty}}+\|\nabla \theta(\cdot, t)\|_{L^{r}}\right)=0
$$

with any $p, r$ as in (1.17). In addition, there exists some positive constant $C$ depending only on $\mu, \lambda, \kappa, R, \gamma, \bar{\rho}$, and $M$, such that, for $\sigma(t) \triangleq \min \{1, t\}$, the following estimates hold

$$
\begin{gathered}
\sup _{t \in(0, \infty)}\|u\|_{H^{1}}+\int_{0}^{\infty} \int\left|(\rho u)_{t}+\operatorname{div}(\rho u \otimes u)\right|^{2} d x d t \leq C, \\
\sup _{t \in(0, \infty)} \int\left((\rho-1)^{2}+\rho|u|^{2}+\rho(\theta-1)^{2}\right) d x \\
\quad+\int_{0}^{\infty}\left(\|\nabla u\|_{L^{2}}^{2}+\|\nabla \theta\|_{L^{2}}^{2}\right) d t \leq C C_{0}^{1 / 4}, \\
\sup _{t \in(0, \infty)}\left(\sigma^{2}\|\nabla u\|_{L^{6}}^{2}+\sigma^{4}\|\theta-1\|_{H^{2}}^{2}\right) \\
+\int_{0}^{\infty}\left(\sigma^{2}\left\|u_{t}\right\|_{L^{2}}^{2}+\sigma^{2}\|\nabla \dot{u}\|_{L^{2}}^{2}+\sigma^{4}\left\|\theta_{t}\right\|_{H^{1}}^{2}\right) d t \leq C C_{0}^{1 / 8} .
\end{gathered}
$$

Moreover, $(\rho, u, \theta)$ satisfies $[1.6)_{3}$ in the weak form, that is, for any test function $\psi \in$ $\mathcal{D}\left(\mathbb{R}^{3} \times(-\infty, \infty)\right)$,

$$
\begin{aligned}
& \frac{R}{\gamma-1} \int \rho_{0} \theta_{0} \psi(\cdot, 0) d x+\frac{R}{\gamma-1} \int_{0}^{\infty} \int \rho \theta\left(\psi_{t}+u \cdot \nabla \psi\right) d x d t \\
& =\kappa \int_{0}^{\infty} \int \nabla \theta \cdot \nabla \psi d x d t+R \int_{0}^{\infty} \int \rho \theta \operatorname{div} u \psi d x d t \\
& \quad-\int_{0}^{\infty} \int\left(\lambda(\operatorname{div} u)^{2}+2 \mu|\mathfrak{D}(u)|^{2}\right) \psi d x d t .
\end{aligned}
$$


The following Corollary 1.3, whose proof can be found in [13, Theorem 1.2], shows that we can obtain from (1.16) the following large time behavior of the gradient of the density when vacuum states appear initially, which is completely in contrast to the classical theory ( [18]).

Corollary 1.3 ( [13]) In addition to the conditions of Theorem 1.1, assume further that there exists some point $x_{0} \in \mathbb{R}^{3}$ such that $\rho_{0}\left(x_{0}\right)=0$. Then the unique global classical solution $(\rho, u, \theta)$ to the Cauchy problem (1.6) (1.4) (1.7) obtained in Theorem 1.1 has to blow up as $t \rightarrow \infty$, in the sense that for any $r>3$,

$$
\lim _{t \rightarrow \infty}\|\nabla \rho(\cdot, t)\|_{L^{r}}=\infty .
$$

A few remarks are in order:

Remark 1.1 It follows from (1.15) that, for any $0<\tau<T<\infty$,

$$
(\rho-1, \nabla \rho, u, \theta-1) \in C\left(\overline{\mathbb{R}^{3}} \times[0, T]\right),
$$

and

$$
\nabla u, \nabla^{2} u \in C\left([\tau, T] ; L^{2}\right) \cap L^{\infty}\left(\tau, T ; W^{1, q}\right) \hookrightarrow C\left(\overline{\mathbb{R}^{3}} \times[\tau, T]\right),
$$

which together with (1.6) 1 and (1.32) gives

$$
\rho_{t} \in C\left(\overline{\mathbb{R}^{3}} \times[\tau, T]\right) .
$$

Similarly, we deduce from (1.15) that

$$
\nabla \theta, \nabla^{2} \theta \in C\left([\tau, T] ; H^{1}\right) \cap L^{\infty}\left(\tau, T ; H^{2}\right) \hookrightarrow C\left(\overline{\mathbb{R}^{3}} \times[\tau, T]\right),
$$

which combining with (1.32)-(1.34) thus shows that the solution $(\rho, u, \theta)$ obtained in Theorem 1.1 is in fact a classical one to the Cauchy problem (1.6) (1.4) (1.7) in $\mathbb{R}^{3} \times$ $(0, \infty)$. Although it has small energy, yet its oscillations could be arbitrarily large. In particular, initial vacuum states are allowed.

Remark 1.2 Theorem 1.1 is the first result concerning the global existence of classical solutions with vacuum to the full compressible Navier-Stokes system. Moreover, the conclusions in Theorem 1.1 generalize the classical theory of Matsumura-Nishida ([18]) to the case of large oscillations since in this case, the requirement of small energy, (1.13), is equivalent to smallness of the mean-square norm of $\left(\rho_{0}-1, u_{0}, \theta_{0}-1\right)$. In addition, the initial density is allowed to vanish and the initial temperature may be zero. However, although the large-time asymptotic behavior (1.16) is similar to that in [18], yet our solution may contain vacuum states, whose appearance leads to the large time blowup behavior stated in Corollary [1.3, this is in sharp contrast to that in [18] where the gradients of the density are suitably small uniformly for all time.

Remark 1.3 It is worth noting that the conclusions in Theorem 1.1 rely heavily on the positivity of both $\tilde{\rho}$ and $\tilde{\theta}$ in (1.4) which prevents the density from being compactly supported or decaying for large values of the spatial variable $x$. Indeed, any smooth solution will blow up in finite time provided that it has compactly supported initial density ( [4, 23]) or that it and its spatial derivatives decay fast enough for large values of the spatial variable $x$ ([21]). Therefore, it would be interesting to study the global existence and large time asymptotic behavior of solutions for the case that initial data decay slowly enough for large values of the spatial variable $x$. This is left for the future. 
Remark 1.4 It should be noted here that Theorem 1.2 is the first result concerning the global existence of weak solutions to (1.1) in the presence of vacuum and extends the global weak solutions of Hoff ([10]) to the case of large oscillations and non-negative initial density. Moreover, the initial temperature is allowed to be zero.

Remark 1.5 It follows from (1.28) and Sobolev's embedding theorem that $u$ and $\theta$ are in fact Hölder continuous away from $t=0$, that is, for any $0<\tau<\infty$,

$$
\sup _{t \in[\tau, \infty)}\|u\|_{L^{\infty}}+\langle u\rangle_{\mathbb{R}^{3} \times[\tau, \infty)}^{1 / 2,1 / 8}+\sup _{t \in[\tau, \infty)}\|\theta\|_{L^{\infty}}+\langle\theta\rangle_{\mathbb{R}^{3} \times[\tau, \infty)}^{1 / 2,1 / 8}<\infty
$$

where we employ the usual notation for Hölder norms:

$$
\langle w\rangle_{Q}^{1 / 2,1 / 8}=\sup _{\substack{(x, t),(y, s) \in Q \\(x, t) \neq(y, s)}} \frac{|w(x, t)-w(y, s)|}{|x-y|^{1 / 2}+|t-s|^{1 / 8}},
$$

for functions $w: Q \subseteq \mathbb{R}^{3} \times[0, \infty) \rightarrow \mathbb{R}^{m}$.

Remark 1.6 Simple calculations yield that if

$$
\sup _{x \in \mathbb{R}^{3}} \theta_{0}(x) \leq \bar{\theta}
$$

we have

$$
\int \rho_{0}\left(\theta_{0}-1\right)^{2} d x \leq 2(\bar{\theta}+1) \int \rho_{0}\left(\theta_{0}-\log \theta_{0}-1\right) d x
$$

which implies $\tilde{C}_{0} \leq C_{0} \leq(\bar{\theta}+2) \tilde{C}_{0}$, where

$$
\begin{aligned}
\tilde{C}_{0} \triangleq & \frac{1}{2} \int \rho_{0}\left|u_{0}\right|^{2} d x+R \int\left(\rho_{0} \log \rho_{0}-\rho_{0}+1\right) d x \\
& +\frac{R}{\gamma-1} \int \rho_{0}\left(\theta_{0}-\log \theta_{0}-1\right) d x
\end{aligned}
$$

is the usual initial energy. In other words, if we replace $C_{0}$ with the usual initial energy $\tilde{C}_{0}$, the $\varepsilon$ in Theorems 1.1 and 1.2 will also depend on the upper bound of the initial temperature.

Remark 1.7 Similar ideas can be applied to study the case on bounded domain. This will be reported in a forthcoming paper [11].

We now comment on the analysis of this paper. Note that though the local existence and uniqueness of strong solutions to (1.6) in the presence of vacuum was obtained by Cho-Kim ( [6]), the local existence of classical solutions with vacuum to (1.6) still remains unknown. Some of the main new difficulties to obtain the classical solutions to (1.6) (1.4) (1.7) for initial data in the class satisfying (1.9)-(1.12) are due to the appearance of vacuum. Thus, we take the strategy that we first extend the standard local classical solutions with strictly positive initial density (see Lemma 2.1) globally in time just under the condition that the initial energy is suitably small (see Proposition 5.1), then let the lower bound of the initial density go to zero. To do so, one needs to establish global a priori estimates, which are independent of the lower bound of the 
density, on smooth solutions to (1.6) (1.4) (1.7) in suitable higher norms. It turns out that the key issue in this paper is to derive both the time-independent upper bound for the density and the time-dependent higher norm estimates of the smooth solution $(\rho, u, \theta)$. Compared to the isentropic case ( [13]), the first main difficulty lies in the fact that the basic energy estimate cannot yield directly the bounds on the $L^{2}$-norm (in both time and space) of the spatial derivatives of both the velocity and the temperature since the super norm of the temperature is just assumed to satisfy the a priori bound $(\min \{1, t\})^{-3 / 2}$ (see $(\underline{3.6})$ ), which in fact could be arbitrarily large for small time. To overcome this difficulty, based on careful analysis on the basic energy estimate, we succeed in deriving a new estimate of the temperature which shows that the spatial $L^{2}$-norm of the deviation of the temperature from its far field value can be bounded by the combination of the initial energy with the spatial $L^{2}$-norm of the spatial derivatives of the temperature (see (3.10) ). This estimate, which will play a crucial role in the analysis of this paper, together with elaborate analysis on the bounds of the energy, then yields the key energy-like estimate, provided that the initial energy is suitably small (see Lemma 3.3). We remark that one of the key issues to obtain such an energylike estimate lies in the positivity of the far field density, which excludes the case of compactly supported initial density.

Next, the second main difficulty is to obtain the time-independent upper bound of the density. Based on careful initial layer analysis and making a full use of the structure of (1.6), we succeed in deriving the weighted spatial mean estimates of the material derivatives of both the velocity and the temperature, which are independent of the lower bound of density, provided that the initial energy is suitably small (see Lemmas 3.4 and 3.5 . This approach is motivated by the basic estimates of the material derivatives of both the velocity and the temperature, which are developed by Hoff ( [10]) in the theory of weak solutions with strictly positive initial density. Having all these estimates at hand, we are able to obtain the desired estimates of $L^{1}\left(0, \min \{1, T\} ; L^{\infty}\left(\mathbb{R}^{3}\right)\right)$-norm and the time-independent ones of $L^{2}\left(\min \{1, T\}, T ; L^{\infty}\left(\mathbb{R}^{3}\right)\right)$-norm of both the effective viscous flux (see (1.21) for the definition) and the deviation of the temperature from its far field value. It follows from these key estimates and a Gronwall-type inequality (see Lemma 2.5) that we are able to obtain a time-uniform upper bound of the density which is crucial for global estimates of classical solutions. This approach to estimate a uniform upper bound for the density is new compared to our previous analysis on the isentropic compressible Navier-Stokes equations in [13].

Then, the third main step is to bound the gradients of the density, the velocity, and the temperature. Motivated by our recent studies ( [12]) on the blow-up criteria of strong (or classical) solutions to the barotropic compressible Navier-Stokes equations, such bounds can be obtained by solving a logarithm Gronwall inequality based on a Beale-Kato-Majda-type inequality (see Lemma 2.6) and the a priori estimates we have just derived. Moreover, such a derivation simultaneously yields the bound for $L^{3 / 2}\left(0, T ; L^{\infty}\left(\mathbb{R}^{3}\right)\right)$-norm of the gradient of the velocity(see Lemma 4.1 and its proof). It should be noted here that we do not require smallness of the gradient of the initial density which prevents the appearance of vacuum ( [18]).

Finally, with these a priori estimates of the gradients of the solutions at hand, one can obtain the desired higher order estimates by careful initial layer analysis on the time derivatives and then the spatial ones of the density, the velocity and the temperature. It should be emphasized here that all these a priori estimates are independent of the lower bound of the density. Therefore, we can build proper approximate solutions with strictly positive initial density then take appropriate limits by letting the lower bound 
of the initial density go to zero. The limiting functions having exactly the desired properties are shown to be the global classical solutions to the Cauchy problem (1.6) (1.4) (1.7). In addition, the initial density is allowed to vanish. We can also establish the global weak solutions almost the same way as we established the classical one with a new modified approximating initial data.

The rest of the paper is organized as follows: In Section 2, we collect some elementary facts and inequalities which will be needed in later analysis. Section 3 is devoted to deriving the lower-order a priori estimates on classical solutions which are needed to extend the local solution to all time. Based on the previous results, higher-order estimates are established in Section 4. Then finally, the main results, Theorems 1.1 and 1.2, are proved in Section 5.

\section{Preliminaries}

The following well-known local existence theory, where the initial density is strictly away from vacuum, can be shown by the standard contraction mapping argument (see for example [18,19], in particular, [18, Theorem 5.2]).

Lemma 2.1 Assume that $\left(\rho_{0}, u_{0}, \theta_{0}\right)$ satisfies

$$
\left(\rho_{0}-1, u_{0}, \theta_{0}-1\right) \in H^{3}, \quad \inf _{x \in \mathbb{R}^{3}} \rho_{0}(x)>0 .
$$

Then there exist a small time $T_{0}>0$ and a unique classical solution $(\rho, u, \theta)$ to the Cauchy problem (1.6) 1.4) 1.7) on $\mathbb{R}^{3} \times\left(0, T_{0}\right.$ ] such that

$$
\begin{gathered}
\inf _{(x, t) \in \mathbb{R}^{3} \times\left(0, T_{0}\right]} \rho(x, t) \geq \frac{1}{2} \inf _{x \in \mathbb{R}^{3}} \rho_{0}(x), \\
\left\{\begin{array}{l}
(\rho-1, u, \theta-1) \in C\left(\left[0, T_{0}\right] ; H^{3}\right), \quad \rho_{t} \in C\left(\left[0, T_{0}\right] ; H^{2}\right), \\
\left(u_{t}, \theta_{t}\right) \in C\left(\left[0, T_{0}\right] ; H^{1}\right), \quad(u, \theta-1) \in L^{2}\left(0, T_{0} ; H^{4}\right),
\end{array}\right.
\end{gathered}
$$

and

$$
\left\{\begin{array}{l}
\left(\sigma u_{t}, \sigma \theta_{t}\right) \in L^{2}\left(0, T_{0} ; H^{3}\right), \quad\left(\sigma u_{t t}, \sigma \theta_{t t}\right) \in L^{2}\left(0, T_{0} ; H^{1}\right), \\
\left(\sigma^{2} u_{t t}, \sigma^{2} \theta_{t t}\right) \in L^{2}\left(0, T_{0} ; H^{2}\right), \quad\left(\sigma^{2} u_{t t t}, \sigma^{2} \theta_{t t t}\right) \in L^{2}\left(0, T_{0} ; L^{2}\right),
\end{array}\right.
$$

where $\sigma(t) \triangleq \min \{1, t\}$. Moreover, for any $(x, t) \in \mathbb{R}^{3} \times\left[0, T_{0}\right]$, the following estimate holds

$$
\theta(x, t) \geq \inf _{x \in \mathbb{R}^{3}} \theta_{0}(x) \exp \left\{-(\gamma-1) \int_{0}^{T_{0}}\|\operatorname{div} u\|_{L^{\infty}} d t\right\},
$$

provided $\inf _{x \in \mathbb{R}^{3}} \theta_{0}(x) \geq 0$.

Proof. We only have to show (2.4) and (2.5), which are not given in [18, Theorem 5.2].

Without loss of generality, assume that $T_{0} \leq 1$. We first prove (2.4) $)_{1}$. Differentiating (1.6) 2 with respect to $t$ leads to

$$
\begin{aligned}
& \rho u_{t t}+\rho_{t} u_{t}+\rho_{t} u \cdot \nabla u+\rho u_{t} \cdot \nabla u+\rho u \cdot \nabla u_{t}+\nabla P_{t} \\
& =\mu \Delta u_{t}+(\mu+\lambda) \nabla \operatorname{div} u_{t} .
\end{aligned}
$$


This shows that $t u_{t}$ satisfies

$$
\left\{\begin{array}{l}
\rho\left(t u_{t}\right)_{t}-\mu \Delta\left(t u_{t}\right)-(\mu+\lambda) \nabla \operatorname{div}\left(t u_{t}\right)=F_{1}, \\
\left(t u_{t}\right)(x, 0)=0
\end{array}\right.
$$

where

$$
F_{1} \triangleq \rho u_{t}-t \rho_{t} u_{t}-t \rho_{t} u \cdot \nabla u-t \rho u_{t} \cdot \nabla u-t \rho u \cdot \nabla u_{t}-R t \nabla\left(\rho_{t} \theta+\rho \theta_{t}\right)
$$

satisfies $F_{1} \in L^{2}\left(0, T_{0} ; L^{2}\right)$ due to (2.3). It thus follows from (2.3), (2.2), and standard $L^{2}$-theory for parabolic system (2.7) that

$$
\left(t u_{t}\right)_{t}, \nabla^{2}\left(t u_{t}\right) \in L^{2}\left(0, T_{0} ; L^{2}\right) .
$$

Similarly, we differentiate $(1.6)_{3}$ with respect to $t$ to get

$$
\begin{aligned}
- & \frac{\kappa(\gamma-1)}{R} \Delta \theta_{t}+\rho \theta_{t t} \\
= & -\rho_{t} \theta_{t}-\rho_{t}(u \cdot \nabla \theta+(\gamma-1) \theta \operatorname{div} u)-\rho(u \cdot \nabla \theta+(\gamma-1) \theta \operatorname{div} u)_{t} \\
& +\frac{\gamma-1}{R}\left(\lambda(\operatorname{div} u)^{2}+2 \mu|\mathfrak{D}(u)|^{2}\right)_{t},
\end{aligned}
$$

which implies that $t \theta_{t}$ satisfies

$$
\left\{\begin{array}{l}
R \rho\left(t \theta_{t}\right)_{t}-\kappa(\gamma-1) \Delta\left(t \theta_{t}\right)=R F_{2} \\
\left(t \theta_{t}\right)(x, 0)=0
\end{array}\right.
$$

with

$$
\begin{aligned}
F_{2} \triangleq & \rho \theta_{t}-t \rho_{t} \theta_{t}-t \rho_{t}(u \cdot \nabla \theta+(\gamma-1) \theta \operatorname{div} u) \\
& \quad-t \rho(u \cdot \nabla \theta+(\gamma-1) \theta \operatorname{div} u)_{t}+\frac{\gamma-1}{R} t\left(\lambda(\operatorname{div} u)^{2}+2 \mu|\mathfrak{D}(u)|^{2}\right)_{t} .
\end{aligned}
$$

One derives from (2.3) that $F_{2} \in L^{2}\left(0, T_{0} ; L^{2}\right)$, which together with (2.3), (2.2), and standard $L^{2}$-theory for parabolic system (2.10) implies

$$
\left(t \theta_{t}\right)_{t}, \nabla^{2}\left(t \theta_{t}\right) \in L^{2}\left(0, T_{0} ; L^{2}\right) .
$$

It thus follows from (2.3), (2.8), and (2.11) that

$$
F_{1}, F_{2} \in L^{2}\left(0, T_{0} ; H^{1}\right),
$$

which together with (2.3), (2.2), (2.7), and (2.10) gives (2.4) 1 .

Next, we prove $(2.4)_{2}$. Differentiating (2.6) with respect to $t$ gives

$$
\begin{aligned}
& \rho u_{t t t}+\rho u \cdot \nabla u_{t t}-\mu \Delta u_{t t}-(\mu+\lambda) \nabla \operatorname{div} u_{t t} \\
& =2 \operatorname{div}(\rho u) u_{t t}+\operatorname{div}(\rho u)_{t} u_{t}-2(\rho u)_{t} \cdot \nabla u_{t}-\left(\rho_{t t} u+2 \rho_{t} u_{t}\right) \cdot \nabla u \\
& \quad-\rho u_{t t} \cdot \nabla u-\nabla P_{t t} .
\end{aligned}
$$

This together with (2.4) 1 and (2.3) implies that $t^{2} u_{t t}$ satisfies

$$
\left\{\begin{array}{l}
\rho\left(t^{2} u_{t t}\right)_{t}-\mu \Delta\left(t^{2} u_{t t}\right)-(\mu+\lambda) \nabla \operatorname{div}\left(t^{2} u_{t t}\right)=F_{3} \\
\left(t^{2} u_{t t}\right)(x, 0)=0
\end{array}\right.
$$


where

$$
\begin{aligned}
F_{3} \triangleq & 2 t \rho u_{t t}-t^{2} \rho u \cdot \nabla u_{t t}+2 t^{2} \operatorname{div}(\rho u) u_{t t}+t^{2} \operatorname{div}(\rho u)_{t} u_{t} \\
& -2 t^{2}(\rho u)_{t} \cdot \nabla u_{t}-t^{2}\left(\rho_{t t} u+2 \rho_{t} u_{t}\right) \cdot \nabla u-t^{2} \rho u_{t t} \cdot \nabla u-t^{2} \nabla P_{t t},
\end{aligned}
$$

satisfies $F_{3} \in L^{2}\left(0, T_{0} ; L^{2}\right)$ due to (2.3) and (2.4) 1 . It follows from (2.2), (2.3), (2.4) 1 , and standard $L^{2}$-estimate for (2.13) that

$$
\left(t^{2} u_{t t}\right)_{t}, \nabla^{2}\left(t^{2} u_{t t}\right) \in L^{2}\left(0, T_{0} ; L^{2}\right) .
$$

Similarly, differentiating (2.9) with respect to $t$ yields

$$
\begin{aligned}
& \rho \theta_{t t t}+\rho u \cdot \nabla \theta_{t t}-\frac{\kappa(\gamma-1)}{R} \Delta \theta_{t t} \\
& =2 \operatorname{div}(\rho u) \theta_{t t}-\rho_{t t}\left(\theta_{t}+u \cdot \nabla \theta+(\gamma-1) \theta \operatorname{div} u\right) \\
& \quad-2 \rho_{t}(u \cdot \nabla \theta+(\gamma-1) \theta \operatorname{div} u)_{t} \\
& \quad-\rho\left(u_{t t} \cdot \nabla \theta+2 u_{t} \cdot \nabla \theta_{t}+(\gamma-1)(\theta \operatorname{div} u)_{t t}\right) \\
& \quad+\frac{\gamma-1}{R}\left(\lambda(\operatorname{div} u)^{2}+2 \mu|\mathfrak{D}(u)|^{2}\right)_{t t} .
\end{aligned}
$$

We thus obtain from (2.4) $1,(2.3)$, and (2.16) that $t^{2} \theta_{t t}$ satisfies

$$
\left\{\begin{array}{l}
R \rho\left(t^{2} \theta_{t t}\right)_{t}-\kappa(\gamma-1) \Delta\left(t^{2} \theta_{t t}\right)=R F_{4}, \\
\left(t^{2} \theta_{t t}\right)(x, 0)=0
\end{array}\right.
$$

with

$$
\begin{aligned}
F_{4} \triangleq & 2 t \rho \theta_{t t}-t^{2} \rho u \cdot \nabla \theta_{t t}+2 t^{2} \operatorname{div}(\rho u) \theta_{t t}-t^{2} \rho_{t t}\left(\theta_{t}+u \cdot \nabla \theta+(\gamma-1) \theta \operatorname{div} u\right) \\
& -2 t^{2} \rho_{t}(u \cdot \nabla \theta+(\gamma-1) \theta \operatorname{div} u)_{t}-t^{2} \rho u_{t t} \cdot \nabla \theta-2 t^{2} \rho u_{t} \cdot \nabla \theta_{t} \\
& -(\gamma-1) t^{2} \rho(\theta \operatorname{div} u)_{t t}+\frac{\gamma-1}{R} t^{2}\left(\lambda(\operatorname{div} u)^{2}+2 \mu|\mathfrak{D}(u)|^{2}\right)_{t t} .
\end{aligned}
$$

It thus follows from (2.3) and (2.4) 1 that $F_{4} \in L^{2}\left(0, T_{0} ; L^{2}\right)$, which together with (2.2), (2.3), (2.4) 1 , and standard $L^{2}$-estimate for (2.17) gives that

$$
\left(t^{2} \theta_{t t}\right)_{t}, \nabla^{2}\left(t^{2} \theta_{t t}\right) \in L^{2}\left(0, T_{0} ; L^{2}\right) .
$$

One thus obtain $(2.4)_{2}$ directly from $(2.3),(2.4) 1,(2.15)$, and (2.18).

Finally, we will show the lower bound of $\theta,(2.5)$, by maximum principle. In fact, it follows from $(1.6)_{3}$ and (1.4) that

$$
\rho \theta_{t}+\rho u \cdot \nabla \theta-\frac{\kappa(\gamma-1)}{R} \Delta \theta+(\gamma-1) \rho \theta \operatorname{div} u \geq 0, \quad \theta \rightarrow 1 \quad \text { as }|x| \rightarrow \infty,
$$

where we have used

$$
2 \mu|\mathfrak{D}(u)|^{2}+\lambda(\operatorname{div} u)^{2} \geq 0
$$

By (2.3), we have

$$
\int_{0}^{T_{0}}\|\operatorname{div} u\|_{L^{\infty}} d t<\infty
$$

which together with the standard maximum principle thus gives (2.5). The proof of Lemma 2.1 is completed.

Next, the following well-known Gagliardo-Nirenberg-Sobolev-type inequality will be used later frequently (see [20]). 
Lemma 2.2 For $p \in(1, \infty)$ and $q \in(3, \infty)$, there exists some generic constant $C>0$ which may depend on $p$ and $q$ such that for $f \in D^{1}\left(\mathbb{R}^{3}\right), g \in L^{p}\left(\mathbb{R}^{3}\right) \cap D^{1, q}\left(\mathbb{R}^{3}\right)$, and $\varphi, \psi \in H^{2}\left(\mathbb{R}^{3}\right)$, we have

$$
\begin{gathered}
\|f\|_{L^{6}} \leq C\|\nabla f\|_{L^{2}} \\
\|g\|_{C\left(\overline{\mathbb{R}^{3}}\right)} \leq C\|g\|_{L^{p}}^{p(q-3) /(3 q+p(q-3))}\|\nabla g\|_{L^{q}}^{3 q /(3 q+p(q-3))} \\
\|\varphi \psi\|_{H^{2}} \leq C\|\varphi\|_{H^{2}}\|\psi\|_{H^{2}}
\end{gathered}
$$

Then, the following inequality is an easy consequence of (2.21) and will be used frequently later.

Lemma 2.3 Let the function $g(x)$ defined in $\mathbb{R}^{3}$ be non-negative and satisfy $g(\cdot)-1 \in$ $L^{2}\left(\mathbb{R}^{3}\right)$. Then there exists a universal positive constant $C$ such that for $r \in[1,2]$ and any open set $\Sigma \subset \mathbb{R}^{3}$, the following estimate holds

$$
\int_{\Sigma}|f|^{r} d x \leq C \int_{\Sigma} g|f|^{r} d x+C\|g-1\|_{L^{2}\left(\mathbb{R}^{3}\right)}^{(6-r) / 3}\|\nabla f\|_{L^{2}\left(\mathbb{R}^{3}\right)}^{r}
$$

for all $f \in\left\{\left.f \in D^{1}\left(\mathbb{R}^{3}\right)|g| f\right|^{r} \in L^{1}(\Sigma)\right\}$.

Proof. In fact, Sobolev's inequality, (2.21), yields that

$$
\begin{aligned}
2 \int_{\Sigma}|f|^{r} d x & \leq 2 \int_{\Sigma} g|f|^{r} d x+2 \int_{\Sigma}|g-1 \| f|^{r} d x \\
& \leq 2 \int_{\Sigma} g|f|^{r} d x+2\|g-1\|_{L^{2}\left(\mathbb{R}^{3}\right)}\|f\|_{L^{r}(\Sigma)}^{r(3-r) /(6-r)}\|f\|_{L^{6}\left(\mathbb{R}^{3}\right)}^{3 r /(6-r)} \\
& \leq 2 \int_{\Sigma} g|f|^{r} d x+\int_{\Sigma}|f|^{r} d x+C\|g-1\|_{L^{2}\left(\mathbb{R}^{3}\right)}^{(6-r) / 3}\|\nabla f\|_{L^{2}\left(\mathbb{R}^{3}\right)}^{r},
\end{aligned}
$$

which implies (2.24) directly.

Next, it follows from (1.6) 2 that $G, \omega$ defined in (1.21) satisfy

$$
\triangle G=\operatorname{div}(\rho \dot{u}), \quad \mu \triangle \omega=\nabla \times(\rho \dot{u}) .
$$

Applying the standard $L^{p}$-estimate to the elliptic systems (2.25) together with (2.21) yields the following elementary estimates (see [13, Lemma 2.3]).

Lemma 2.4 Let $(\rho, u, \theta)$ be a smooth solution of (1.6) (1.4). Then there exists a generic positive constant $C$ depending only on $\mu, \lambda$, and $R$ such that, for any $p \in[2,6]$,

$$
\begin{gathered}
\|\nabla u\|_{L^{p}} \leq C\left(\|G\|_{L^{p}}+\|\omega\|_{L^{p}}\right)+C\|\rho \theta-1\|_{L^{p}}, \\
\|\nabla G\|_{L^{p}}+\|\nabla \omega\|_{L^{p}} \leq C\|\rho \dot{u}\|_{L^{p}}, \\
\|G\|_{L^{p}}+\|\omega\|_{L^{p}} \leq C\|\rho \dot{u}\|_{L^{2}}^{(3 p-6) /(2 p)}\left(\|\nabla u\|_{L^{2}}+\|\rho \theta-1\|_{L^{2}}\right)^{(6-p) /(2 p)}, \\
\|\nabla u\|_{L^{p}} \leq C\|\nabla u\|_{L^{2}}^{(6-p) /(2 p)}\left(\|\rho \dot{u}\|_{L^{2}}+\|\rho \theta-1\|_{L^{6}}\right)^{(3 p-6) /(2 p)} .
\end{gathered}
$$

Next, the following Gronwall-type inequality will be used to get the uniform (in time) upper bound of the density $\rho$. 
Lemma 2.5 Let the function $y \in W^{1,1}(0, T)$ satisfy

$$
y^{\prime}(t)+\alpha y(t) \leq g(t) \text { on }[0, T], \quad y(0)=y^{0},
$$

where $\alpha$ is a positive constant and $g \in L^{p}\left(0, T_{1}\right) \cap L^{q}\left(T_{1}, T\right)$ for some $p \geq 1, q \geq 1$, and $T_{1} \in[0, T]$. Then

$$
\sup _{0 \leq t \leq T} y(t) \leq\left|y^{0}\right|+\left(1+\alpha^{-1}\right)\left(\|g\|_{L^{p}\left(0, T_{1}\right)}+\|g\|_{L^{q}\left(T_{1}, T\right)}\right) .
$$

Proof. Let $p^{\prime}$ and $q^{\prime}$ denote the conjugate numbers of $p$ and $q$ respectively. Multiplying (2.30) by $e^{\alpha t}$ and integrating the resulting inequality over $(0, t)$ yield that

$$
\begin{aligned}
e^{\alpha t} y(t) & \leq y^{0}+\int_{0}^{t} e^{\alpha s} g(s) d s \\
& \leq y^{0}+\int_{0}^{\min \left\{t, T_{1}\right\}} e^{\alpha s}|g(s)| d s+\int_{\min \left\{t, T_{1}\right\}}^{t} e^{\alpha s}|g(s)| d s \\
& \leq\left|y^{0}\right|+\|g\|_{L^{p}\left(0, \min \left\{t, T_{1}\right\}\right)}\left\|e^{\alpha s}\right\|_{L^{p^{\prime}(0, t)}}+\|g\|_{L^{q}\left(\min \left\{t, T_{1}\right\}, t\right)}\left\|e^{\alpha s}\right\|_{L^{q^{\prime}}(0, t)} \\
& \leq\left|y^{0}\right|+\left(\|g\|_{L^{p}\left(0, T_{1}\right)}+\|g\|_{L^{q}\left(T_{1}, T\right)}\right)\left(1+\alpha^{-1}\right) e^{\alpha t},
\end{aligned}
$$

where in the last inequality we have used the following simple fact:

$$
\left\|e^{\alpha s}\right\|_{L^{r}(0, t)} \leq\left(1+\alpha^{-1}\right) e^{\alpha t}
$$

for all $r \in[1, \infty]$. We thus derive (2.31) directly from (2.32) and finish the proof of Lemma 2.5.

Finally, we state the following Beale-Kato-Majda-type inequality whose proof can be found in [2,12] and will be used later to estimate $\|\nabla u\|_{L^{\infty}}$ and $\|\nabla \rho\|_{L^{2} \cap L^{6}}$.

Lemma 2.6 ( [2, 12]) For $3<q<\infty$, there is a constant $C(q)$ such that the following estimate holds for all $\nabla u \in L^{2}\left(\mathbb{R}^{3}\right) \cap D^{1, q}\left(\mathbb{R}^{3}\right)$ :

$$
\begin{aligned}
\|\nabla u\|_{L^{\infty}\left(\mathbb{R}^{3}\right)} \leq & C\left(\|\operatorname{div} u\|_{L^{\infty}\left(\mathbb{R}^{3}\right)}+\|\nabla \times u\|_{L^{\infty}\left(\mathbb{R}^{3}\right)}\right) \log \left(e+\left\|\nabla^{2} u\right\|_{L^{q}\left(\mathbb{R}^{3}\right)}\right) \\
& +C\|\nabla u\|_{L^{2}\left(\mathbb{R}^{3}\right)}+C
\end{aligned}
$$

\section{A priori estimates (I): Lower-order estimates}

In this section, we will establish a priori bounds for the smooth, local-in-time solution to (1.6) (1.4) (1.7) obtained in Lemma 2.1. We thus fix a smooth solution $(\rho, u, \theta)$ of (1.6) (1.4) (1.7) on $\mathbb{R}^{3} \times(0, T]$ for some time $T>0$, with initial data $\left(\rho_{0}, u_{0}, \theta_{0}\right)$ satisfying (2.1).

For $\sigma(t) \triangleq \min \{1, t\}$, we define $A_{i}(T)(i=1, \cdots, 4)$ as follows:

$$
\begin{gathered}
A_{1}(T)=\sup _{t \in[0, T]}\|\nabla u\|_{L^{2}}^{2}+\int_{0}^{T} \int \rho|\dot{u}|^{2} d x d t, \\
A_{2}(T)=\frac{R}{2(\gamma-1)} \sup _{t \in[0, T]} \int \rho(\theta-1)^{2} d x+\int_{0}^{T}\left(\|\nabla u\|_{L^{2}}^{2}+\|\nabla \theta\|_{L^{2}}^{2}\right) d t,
\end{gathered}
$$




$$
\begin{array}{r}
A_{3}(T)=\sup _{t \in(0, T]}\left(\sigma\|\nabla u\|_{L^{2}}^{2}+\sigma^{2} \int \rho|\dot{u}|^{2} d x+\sigma^{2}\|\nabla \theta\|_{L^{2}}^{2}\right) \\
+\int_{0}^{T} \int\left(\sigma \rho|\dot{u}|^{2}+\sigma^{2}|\nabla \dot{u}|^{2}+\sigma^{2} \rho(\dot{\theta})^{2}\right) d x d t \\
A_{4}(T)=\sup _{t \in(0, T]} \sigma^{4} \int \rho|\dot{\theta}|^{2} d x+\int_{0}^{T} \int \sigma^{4}|\nabla \dot{\theta}|^{2} d x d t .
\end{array}
$$

We have the following key a priori estimates on $(\rho, u, \theta)$.

Proposition 3.1 For given numbers $M>0$ (not necessarily small), and $\bar{\rho}>2$, assume that $\left(\rho_{0}, u_{0}, \theta_{0}\right)$ satisfies

$$
0<\inf _{x \in \mathbb{R}^{3}} \rho_{0}(x) \leq \sup _{x \in \mathbb{R}^{3}} \rho_{0}(x)<\bar{\rho}, \quad \inf _{x \in \mathbb{R}^{3}} \theta_{0}(x)>0, \quad\left\|\nabla u_{0}\right\|_{L^{2}} \leq M .
$$

Then there exist positive constants $K$ and $\varepsilon_{0}$ both depending only on $\mu, \lambda, \kappa, R, \gamma, \bar{\rho}$, and $M$ such that if $(\rho, u, \theta)$ is a smooth solution of (1.6) (1.4) [1.7) on $\mathbb{R}^{3} \times(0, T]$ satisfying

$$
0<\rho \leq 2 \bar{\rho}, \quad A_{1}(\sigma(T)) \leq 3 K, \quad A_{i}(T) \leq 2 C_{0}^{1 /(2 i)}(i=2,3,4)
$$

the following estimates hold

$$
0<\rho \leq 3 \bar{\rho} / 2, \quad A_{1}(\sigma(T)) \leq 2 K, \quad A_{i}(T) \leq C_{0}^{1 /(2 i)}(i=2,3,4),
$$

provided

$$
C_{0} \leq \varepsilon_{0}
$$

Proof. Proposition 3.1 is an easy consequence of the following Lemmas 3.2, 3.3, and 3.6 3.8, with $\varepsilon_{0}$ as in (3.101).

In this section, we let $C$ denote some generic positive constant depending only on $\mu, \lambda, \kappa, R, \gamma, \bar{\rho}$, and $M$, and we write $C(\alpha)$ to emphasize that $C$ may depend on $\alpha$.

The following elementary $L^{2}$ bounds are crucial for deriving the desired estimate on $A_{2}(T)$ (see Lemma 3.3 below).

Lemma 3.1 Under the conditions of Proposition 3.1, there exists a positive constant $C=C(\bar{\rho})$ depending only on $\mu, \lambda, \kappa, R, \gamma$, and $\bar{\rho}$ such that if $(\rho, u, \theta)$ is a smooth solution of (1.6) 1.4) 1.7) on $\mathbb{R}^{3} \times(0, T]$ satisfying $0<\rho \leq 2 \bar{\rho}$, the following estimates hold

$$
\sup _{0 \leq t \leq T} \int\left(\rho|u|^{2}+(\rho-1)^{2}\right) d x \leq C(\bar{\rho}) C_{0},
$$

and

$$
\|(\theta-1)(\cdot, t)\|_{L^{2}} \leq C(\bar{\rho}) C_{0}^{1 / 2}+C(\bar{\rho}) C_{0}^{1 / 3}\|\nabla \theta(\cdot, t)\|_{L^{2}},
$$

for all $t \in(0, T]$.

Proof. First, it follows from (3.5) and (2.5) that, for all $(x, t) \in \mathbb{R}^{3} \times(0, T)$,

$$
\theta(x, t)>0
$$


Adding $(\underline{1.6})_{2}$ multiplied by $u$ to $(\underline{1.6})_{3}$ multiplied by $1-\theta^{-1}$, we obtain after integrating the resulting equality over $\mathbb{R}^{3}$ and using $(1.6)_{1}$ that

$$
\begin{aligned}
\frac{d}{d t} \int\left(\frac{1}{2} \rho|u|^{2}+R(1+\rho \log \rho-\rho)+\frac{R}{\gamma-1} \rho(\theta-\log \theta-1)\right) d x \\
=\int\left[-\mu|\nabla u|^{2}-(\lambda+\mu)(\operatorname{div} u)^{2}-\kappa \theta^{-2}|\nabla \theta|^{2}\right. \\
\left.\quad+\left(1-\theta^{-1}\right)\left(\lambda(\operatorname{div} u)^{2}+2 \mu|\mathfrak{D}(u)|^{2}\right)\right] d x \\
=-\int\left(\theta^{-1}\left(\lambda(\operatorname{div} u)^{2}+2 \mu|\mathfrak{D}(u)|^{2}\right)+\kappa \theta^{-2}|\nabla \theta|^{2}\right) d x,
\end{aligned}
$$

where in the second equality we have used

$$
2 \int|\mathfrak{D}(u)|^{2} d x=\int\left(|\nabla u|^{2}+(\operatorname{div} u)^{2}\right) d x .
$$

Direct calculations yield that

$$
\begin{aligned}
\rho \log \rho-\rho+1 & =(\rho-1)^{2} \int_{0}^{1} \frac{1-\alpha}{\alpha(\rho-1)+1} d \alpha \\
& \geq \frac{(\rho-1)^{2}}{\bar{\rho}} \int_{0}^{1}(1-\alpha) d \alpha \\
& =\frac{1}{2 \bar{\rho}}(\rho-1)^{2},
\end{aligned}
$$

and

$$
\begin{aligned}
\theta-\log \theta-1 & =(\theta-1)^{2} \int_{0}^{1} \frac{\alpha}{\alpha(\theta-1)+1} d \alpha \\
& \geq \frac{1}{8}(\theta-1) 1_{(\theta(\cdot, t)>2)}+\frac{1}{12}(\theta-1)^{2} 1_{(\theta(\cdot, t)<3)},
\end{aligned}
$$

where we denote

$$
(\theta(\cdot, t)>2) \triangleq\left\{x \in \mathbb{R}^{3} \mid \theta(x, t)>2\right\}, \quad(\theta(\cdot, t)<3) \triangleq\left\{x \in \mathbb{R}^{3} \mid \theta(x, t)<3\right\} .
$$

Integrating (3.12) with respect to $t$ over $(0, T)$ yields that

$$
\begin{aligned}
& \sup _{0 \leq t \leq T} \int\left(\frac{1}{2} \rho|u|^{2}+R(1+\rho \log \rho-\rho)+\frac{R}{\gamma-1} \rho(\theta-\log \theta-1)\right) d x \\
& +\int_{0}^{T} \int\left(\frac{1}{\theta}\left(\lambda(\operatorname{div} u)^{2}+2 \mu|\mathfrak{D}(u)|^{2}\right)+\kappa \frac{|\nabla \theta|^{2}}{\theta^{2}}\right) d x d t \leq C_{0},
\end{aligned}
$$

which together with (2.20), (3.11), (3.14), and (3.15) leads to

$$
\begin{aligned}
& \sup _{0 \leq t \leq T} \int\left(\rho|u|^{2}+(\rho-1)^{2}\right) d x \\
& +\sup _{0 \leq t \leq T} \int\left(\rho(\theta-1) 1_{(\theta(\cdot, t)>2)}+\rho(\theta-1)^{2} 1_{(\theta(\cdot, t)<3)}\right) d x \leq C(\bar{\rho}) C_{0} .
\end{aligned}
$$

This fact gives (3.9) directly.

Next, we shall prove (3.10). Taking $g(x)=\rho(x, t), f(x)=\theta(x, t)-1, r=2$ and $\Sigma=(\theta(\cdot, t)<3)$ in (2.24), we conclude after using (3.17) that

$$
\|\theta(\cdot, t)-1\|_{L^{2}(\theta(\cdot, t)<3)} \leq C(\bar{\rho}) C_{0}^{1 / 2}+C(\bar{\rho}) C_{0}^{1 / 3}\|\nabla \theta(\cdot, t)\|_{L^{2}\left(\mathbb{R}^{3}\right)} .
$$


Similarly, taking $g(x)=\rho(x, t), f(x)=\theta(x, t)-1, r=1$ and $\Sigma=(\theta(\cdot, t)>2)$ in (2.24), we obtain after using (3.17) that

$$
\|\theta(\cdot, t)-1\|_{L^{1}(\theta(\cdot, t)>2)} \leq C(\bar{\rho}) C_{0}+C(\bar{\rho}) C_{0}^{5 / 6}\|\nabla \theta(\cdot, t)\|_{L^{2}\left(\mathbb{R}^{3}\right)},
$$

which together with Hölder's inequality and (2.21) leads to

$$
\begin{aligned}
& \|\theta(\cdot, t)-1\|_{L^{2}(\theta(\cdot, t)>2)} \\
& \leq\|\theta(\cdot, t)-1\|_{L^{1}(\theta(\cdot, t)>2)}^{2 / 5}\|\theta(\cdot, t)-1\|_{L^{6}\left(\mathbb{R}^{3}\right)}^{3 / 5} \\
& \leq C(\bar{\rho})\left(C_{0}^{2 / 5}+C_{0}^{1 / 3}\|\nabla \theta(\cdot, t)\|_{L^{2}}^{2 / 5}\right)\|\nabla \theta(\cdot, t)\|_{L^{2}}^{3 / 5} \\
& \leq C(\bar{\rho}) C_{0}^{1 / 2}+C(\bar{\rho}) C_{0}^{1 / 3}\|\nabla \theta(\cdot, t)\|_{L^{2}} .
\end{aligned}
$$

Because of $\mathbb{R}^{3}=(\theta(\cdot, t)<3) \cup(\theta(\cdot, t)>2)$, the combination of (3.18) with (3.19) yields (3.10) directly. We finish the proof of Lemma 3.1.

Next, the following lemma will give an estimate on the term $A_{1}(\sigma(T))$.

Lemma 3.2 Under the conditions of Proposition 3.1, there exist positive constants $K \geq M+1$ and $\varepsilon_{1} \leq 1$ both depending only on $\mu, \lambda, \kappa, R, \gamma, \bar{\rho}$, and $M$ such that if $(\rho, u, \theta)$ is a smooth solution of (1.6) (1.4) 1.7) on $\mathbb{R}^{3} \times(0, T]$ satisfying

$$
0<\rho \leq 2 \bar{\rho}, \quad A_{2}(\sigma(T)) \leq 2 C_{0}^{1 / 4}
$$

the following estimate holds

$$
A_{1}(\sigma(T)) \leq 2 K
$$

provided $A_{1}(\sigma(T)) \leq 3 K$ and $C_{0} \leq \varepsilon_{1}$.

Proof. First, multiplying $(1.6)_{2}$ by $2 u_{t}$, integrating the resulting equality over $\mathbb{R}^{3}$, we obtain after integration by parts that

$$
\begin{aligned}
& \frac{d}{d t} \int\left(\mu|\nabla u|^{2}+(\mu+\lambda)(\operatorname{div} u)^{2}\right) d x+\int \rho\left|u_{t}\right|^{2} d x \\
& \leq-2 \int \nabla P \cdot u_{t} d x+\int \rho|u \cdot \nabla u|^{2} d x \\
& =2 R \frac{d}{d t} \int(\rho \theta-1) \operatorname{div} u d x-2 \int P_{t} \operatorname{div} u d x+\int \rho|u \cdot \nabla u|^{2} d x \\
& =2 R \frac{d}{d t} \int(\rho \theta-1) \operatorname{div} u d x-\frac{R^{2}}{2 \mu+\lambda} \frac{d}{d t} \int(\rho \theta-1)^{2} d x \\
& \quad-\frac{2}{2 \mu+\lambda} \int P_{t} G d x+\int \rho|u \cdot \nabla u|^{2} d x
\end{aligned}
$$

where in the last equality, we have used

$$
\operatorname{div} u=\frac{1}{2 \mu+\lambda}(G+R(\rho \theta-1))
$$

due to (1.21).

Next, assume that $C_{0} \leq 1$. It follows from Hölder's inequality, (3.20), (2.21), and (3.9) that for $p \in[2,6]$,

$$
\begin{aligned}
\|\rho \theta-1\|_{L^{p}} & =\|\rho(\theta-1)+(\rho-1)\|_{L^{p}} \\
& \leq\|\rho(\theta-1)\|_{L^{2}}^{(6-p) /(2 p)}\|\rho(\theta-1)\|_{L^{6}}^{3(p-2) /(2 p)}+\|\rho-1\|_{L^{p}} \\
& \leq C(\bar{\rho}) C_{0}^{(6-p) /(16 p)}\|\nabla \theta\|_{L^{2}}^{3(p-2) /(2 p)}+C(\bar{\rho}) C_{0}^{1 / p},
\end{aligned}
$$


which together with (2.29) yields

$$
\|\nabla u\|_{L^{6}} \leq C(\bar{\rho})\left(\left\|\rho^{1 / 2} \dot{u}\right\|_{L^{2}}+\|\nabla \theta\|_{L^{2}}+C_{0}^{1 / 6}\right) .
$$

Noticing that (1.6) implies

$$
\begin{aligned}
P_{t}= & -\operatorname{div}(P u)-(\gamma-1) P \operatorname{div} u+(\gamma-1) \kappa \Delta \theta \\
& +(\gamma-1)\left(\lambda(\operatorname{div} u)^{2}+2 \mu|\mathfrak{D}(u)|^{2}\right),
\end{aligned}
$$

we obtain after integration by parts and using (3.20), (2.21), (2.27), (3.25), (3.24), and (3.9) that

$$
\begin{aligned}
& \left|\int P_{t} G d x\right| \\
\leq & C \int P(|G||\nabla u|+|u \| \nabla G|) d x+\int\left(|\nabla \theta \| \nabla G|+|\nabla u|^{2}|G|\right) d x \\
\leq & C \int \rho(|G||\nabla u|+|u \| \nabla G|) d x+C \int \rho|\theta-1|(|G||\nabla u|+|u \| \nabla G|) d x \\
& +C\|\nabla G\|_{L^{2}}\|\nabla \theta\|_{L^{2}}+C\|\nabla G\|_{L^{2}}\|\nabla u\|_{L^{2}}^{3 / 2}\|\nabla u\|_{L^{6}}^{1 / 2} \\
\leq & C(\bar{\rho})\left(\|\nabla u\|_{L^{2}}+\|\rho \theta-1\|_{L^{2}}\right)\|\nabla u\|_{L^{2}}+C\|\rho u\|_{L^{2}}\|\nabla G\|_{L^{2}} \\
& +C(\bar{\rho})\|\rho(\theta-1)\|_{L^{2}}^{1 / 2}\|\nabla \theta\|_{L^{2}}^{1 / 2}\|\nabla G\|_{L^{2}}\|\nabla u\|_{L^{2}}+C\|\nabla G\|_{L^{2}}\|\nabla \theta\|_{L^{2}} \\
& +C(\bar{\rho})\|\nabla G\|_{L^{2}}\|\nabla u\|_{L^{2}}^{3 / 2}\left(\|\rho \dot{u}\|_{L^{2}}^{1 / 2}+\|\nabla \theta\|_{L^{2}}^{1 / 2}+C_{0}^{1 / 12}\right) \\
\leq & C(\delta, \bar{\rho}) C_{0}^{1 / 4}+C(\bar{\rho}, \delta)\|\nabla u\|_{L^{2}}^{2}+\delta\|\nabla G\|_{L^{2}}^{2}+C(\bar{\rho}, \delta)\|\nabla \theta\|_{L^{2}}\|\nabla u\|_{L^{2}}^{2} \\
& +C(\delta, \bar{\rho})\|\nabla \theta\|_{L^{2}}^{2}+\delta\left\|\rho^{1 / 2} \dot{u}\right\|_{L^{2}}^{2}+C(\delta, \bar{\rho})\|\nabla u\|_{L^{2}}^{6} \\
\leq & C(\bar{\rho}) \delta\left\|\rho^{1 / 2} \dot{u}\right\|_{L^{2}}^{2}+C(\delta, \bar{\rho})\left(\|\nabla \theta\|_{L^{2}}^{2}+\|\nabla u\|_{L^{2}}^{2}+1\right)+C(\delta, \bar{\rho})\|\nabla u\|_{L^{2}}^{6}
\end{aligned}
$$

Finally, it follows from (2.21) and (3.25) that

$$
\begin{aligned}
& \int \rho|u \cdot \nabla u|^{2} d x \\
& \leq C(\bar{\rho})\|u\|_{L^{6}}^{2}\|\nabla u\|_{L^{2}}\|\nabla u\|_{L^{6}} \\
& \leq C(\bar{\rho})\|\nabla u\|_{L^{2}}^{3}\left(\|\rho \dot{u}\|_{L^{2}}+\|\nabla \theta\|_{L^{2}}+C_{0}^{1 / 6}\right) \\
& \leq \delta\left\|\rho^{1 / 2} \dot{u}\right\|_{L^{2}}^{2}+C(\bar{\rho}, \delta)\|\nabla u\|_{L^{2}}^{6}+C(\bar{\rho}, \delta)\left(\|\nabla u\|_{L^{2}}^{2}+\|\nabla \theta\|_{L^{2}}^{2}\right),
\end{aligned}
$$

where we have used

$$
\|\nabla u\|_{L^{2}}^{3} \leq\|\nabla u\|_{L^{2}}^{2}+\|\nabla u\|_{L^{2}}^{6} .
$$

Substituting (3.27) and (3.28) into (3.22), choosing $\delta$ suitably small, we get after integrating (3.22) over $(0, \sigma(T))$ and using (3.20) that

$$
\begin{aligned}
& \sup _{0 \leq t \leq \sigma(T)}\|\nabla u\|_{L^{2}}^{2}+\int_{0}^{\sigma(T)} \int \rho|\dot{u}|^{2} d x d t \\
& \leq C M+C(\bar{\rho}) C_{0}^{1 / 4}+C(\bar{\rho}) C_{0}^{1 / 4} \sup _{0 \leq t \leq \sigma(T)}\|\nabla u\|_{L^{2}}^{4} \\
& \leq K+C(\bar{\rho}) C_{0}^{1 / 4} \sup _{0 \leq t \leq \sigma(T)}\|\nabla u\|_{L^{2}}^{4},
\end{aligned}
$$


where $K$ is defined by

$$
K \triangleq C M+C(\bar{\rho})+1,
$$

depending only on $\mu, \lambda, \kappa, R, \gamma, \bar{\rho}$, and $M$. We thus finish the proof of (3.21) by choosing $\varepsilon_{1} \triangleq \min \left\{1,(9 C(\bar{\rho}) K)^{-4}\right\}$. The proof of Lemma 3.2 is completed.

Next, the following energy-like estimate of the local smooth solution will play a key role in obtaining further estimates.

Lemma 3.3 Under the conditions of Proposition 3.1, there exists a positive constant $\varepsilon_{2}$ depending only on $\mu, \lambda, \kappa, R, \gamma, \bar{\rho}$, and $M$ such that if $(\rho, u, \theta)$ is a smooth solution of (1.6) (1.4) (1.7) on $\mathbb{R}^{3} \times(0, T]$ satisfying (3.6) with $K$ as in Lemma 3.2, the following estimate holds

$$
A_{2}(T) \leq C_{0}^{1 / 4}
$$

provided $C_{0} \leq \varepsilon_{2}$.

Proof. First, assume that $C_{0} \leq 1$. Multiplying (1.6) 2 by $u$ and integrating the resulting equality over $\mathbb{R}^{3}$ give

$$
\begin{aligned}
& \frac{d}{d t} \int\left(\frac{1}{2} \rho|u|^{2}+R(1+\rho \log \rho-\rho)\right) d x \\
& \quad+\mu \int|\nabla u|^{2} d x+(\mu+\lambda) \int(\operatorname{div} u)^{2} d x \\
& \leq C(\bar{\rho})\left(\|\theta-1\|_{L^{2}}+\|\rho-1\|_{L^{2}}\right)\|\nabla u\|_{L^{2}} \\
& \leq C(\bar{\rho})\left(C_{0}^{1 / 2}+C_{0}^{1 / 3}\|\nabla \theta\|_{L^{2}}\right)\|\nabla u\|_{L^{2}} \\
& \leq C(\bar{\rho}) C_{0}^{2 / 3}+C(\bar{\rho}) C_{0}^{1 / 3}\left(\|\nabla \theta\|_{L^{2}}^{2}+\|\nabla u\|_{L^{2}}^{2}\right),
\end{aligned}
$$

where in the second inequality we have used (3.9) and (3.10).

Multiplying (1.6) $)_{3}$ by $\theta-1$ and integrating the resulting equality over $\mathbb{R}^{3}$ lead to

$$
\begin{aligned}
& \frac{R}{2(\gamma-1)} \frac{d}{d t} \int \rho(\theta-1)^{2} d x+\kappa\|\nabla \theta\|_{L^{2}}^{2} \\
& \leq C(\bar{\rho}) \int \theta|\theta-1||\operatorname{div} u| d x+C \int|\nabla u|^{2}|\theta-1| d x .
\end{aligned}
$$

For the first term on the righthand side of (3.33), we have

$$
\begin{aligned}
& \int \theta|\theta-1 \| \operatorname{div} u| d x \\
& \leq \int(\theta-1)^{2}|\operatorname{div} u| d x+\int|\theta-1 \| \operatorname{div} u| d x \\
& \leq C\|\theta-1\|_{L^{2}}^{1 / 2}\|\theta-1\|_{L^{6}}^{3 / 2}\|\nabla u\|_{L^{2}}+C\|\theta-1\|_{L^{2}}\|\nabla u\|_{L^{2}} \\
& \leq C(\bar{\rho}, M)\left(C_{0}^{1 / 4}+C_{0}^{1 / 6}\|\nabla \theta\|_{L^{2}}^{1 / 2}\right)\|\nabla \theta\|_{L^{2}}^{3 / 2} \\
& \quad+C(\bar{\rho})\left(C_{0}^{1 / 2}+C_{0}^{1 / 3}\|\nabla \theta\|_{L^{2}}\right)\|\nabla u\|_{L^{2}} \\
& \leq C(\bar{\rho}, M) C_{0}^{1 / 2}+C(\bar{\rho}, M) C_{0}^{1 / 6}\left(\|\nabla \theta\|_{L^{2}}^{2}+\|\nabla u\|_{L^{2}}^{2}\right),
\end{aligned}
$$

where we have used (2.21), (3.10), (3.6), and the following simple fact:

$$
\sup _{t \in[0, T]}\|\nabla u\|_{L^{2}} \leq A_{1}(\sigma(T))+A_{3}(T) \leq C(\bar{\rho}, M),
$$


due to (3.6). For the second one on the righthand side of (3.33), in light of (3.10), (2.21), (2.29), (3.25), and (3.6), we have

$$
\begin{aligned}
& \int|\nabla u|^{2}|\theta-1| d x \\
& \leq C\|\theta-1\|_{L^{2}}^{1 / 2}\|\theta-1\|_{L^{6}}^{1 / 2}\|\nabla u\|_{L^{2}}\|\nabla u\|_{L^{6}} \\
& \leq C(\bar{\rho}, M)\left(C_{0}^{1 / 4}\|\nabla \theta\|_{L^{2}}^{1 / 2}+C_{0}^{1 / 6}\|\nabla \theta\|_{L^{2}}\right)\left(\|\rho \dot{u}\|_{L^{2}}+\|\nabla \theta\|_{L^{2}}+C_{0}^{1 / 6}\right) \\
& \leq C(\bar{\rho}, M, \delta) C_{0}^{1 / 3}\left(\left\|\rho^{1 / 2} \dot{u}\right\|_{L^{2}}^{2}+1\right)+C(\bar{\rho}, M)\left(\delta+C_{0}^{1 / 6}\right)\|\nabla \theta\|_{L^{2}}^{2} .
\end{aligned}
$$

Substituting (3.34) and (3.36) into (3.33) leads to

$$
\begin{aligned}
& \frac{R}{2(\gamma-1)} \frac{d}{d t} \int \rho(\theta-1)^{2} d x+\kappa\|\nabla \theta\|_{L^{2}}^{2} \\
& \leq C(\bar{\rho}, M)\left(\delta+C_{0}^{1 / 6}\right)\left(\|\nabla \theta\|_{L^{2}}^{2}+\|\nabla u\|_{L^{2}}^{2}\right) \\
& \quad+C(\bar{\rho}, M, \delta) C_{0}^{1 / 3}\left(\left\|\rho^{1 / 2} \dot{u}\right\|_{L^{2}}^{2}+1\right) .
\end{aligned}
$$

The combination of (3.32) with (3.37) yields

$$
\begin{aligned}
& \frac{d}{d t} \int\left(\frac{1}{2} \rho|u|^{2}+R(1+\rho \log \rho-\rho)+\frac{R}{2(\gamma-1)} \rho(\theta-1)^{2}\right) d x \\
& +\mu \int|\nabla u|^{2} d x+(\mu+\lambda) \int(\operatorname{div} u)^{2} d x+\kappa \int|\nabla \theta|^{2} d x \\
& \leq C(\bar{\rho}, M)\left(\delta+C_{0}^{1 / 6}\right)\left(\|\nabla \theta\|_{L^{2}}^{2}+\|\nabla u\|_{L^{2}}^{2}\right) \\
& \quad+C(\bar{\rho}, M, \delta) C_{0}^{1 / 3}\left(\left\|\rho^{1 / 2} \dot{u}\right\|_{L^{2}}^{2}+1\right) .
\end{aligned}
$$

We assume that

$$
C_{0} \leq \varepsilon_{2}^{1} \triangleq \min \left\{1,\left((4 C(\bar{\rho}, M))^{-1} \min \{\mu, \kappa\}\right)^{6}\right\} .
$$

Choosing $\delta \leq(4 C(\bar{\rho}, M))^{-1} \min \{\mu, \kappa\}$ and integrating (3.38) over $(0, \sigma(T))$, we obtain after using (3.6) that

$$
\begin{aligned}
& \sup _{0 \leq t \leq \sigma(T)} \int\left(\rho|u|^{2}+(\rho-1)^{2}+\frac{R}{2(\gamma-1)} \rho(\theta-1)^{2}\right) d x \\
& +\int_{0}^{\sigma(T)}\left(\|\nabla u\|_{L^{2}}^{2}+\|\nabla \theta\|_{L^{2}}^{2}\right) d t \leq C(\bar{\rho}, M) C_{0}^{1 / 3} .
\end{aligned}
$$

Next, applying the standard $L^{2}$-estimate to the following elliptic problem

$$
\left\{\begin{array}{l}
\kappa \Delta \theta=\frac{R}{\gamma-1} \rho \dot{\theta}+R \rho \theta \operatorname{div} u-\lambda(\operatorname{div} u)^{2}-2 \mu|\mathfrak{D}(u)|^{2}, \\
\theta \rightarrow 1 \quad \text { as }|x| \rightarrow \infty
\end{array}\right.
$$

gives

$$
\begin{aligned}
\left\|\nabla^{2} \theta\right\|_{L^{2}}^{2} \leq & C\left(\|\rho \dot{\theta}\|_{L^{2}}^{2}+\|\nabla u\|_{L^{4}}^{4}+\|\theta \nabla u\|_{L^{2}}^{2}\right) \\
\leq & C(\bar{\rho})\left(\|\nabla \theta\|_{L^{2}}^{2}+\|\nabla u\|_{L^{2}}^{2}\right)\left(\left\|\rho^{1 / 2} \dot{u}\right\|_{L^{2}}^{2}+\|\nabla \theta\|_{L^{2}}^{2}+1\right) \\
& +C\left(\|\rho \dot{\theta}\|_{L^{2}}^{2}+\|\nabla u\|_{L^{4}}^{4}\right)
\end{aligned}
$$


where we have used

$$
\begin{aligned}
& \int \theta^{2}|\nabla u|^{2} d x \\
& \leq C\|\theta-1\|_{L^{6}}^{2}\|\nabla u\|_{L^{2}}\|\nabla u\|_{L^{6}}+C\|\nabla u\|_{L^{2}}^{2} \\
& \leq C(\bar{\rho})\left(\|\nabla \theta\|_{L^{2}}^{2}+\|\nabla u\|_{L^{2}}^{2}\right)\left(\left\|\rho^{1 / 2} \dot{u}\right\|_{L^{2}}^{2}+\|\nabla \theta\|_{L^{2}}^{2}+1\right),
\end{aligned}
$$

due to (2.21) and (3.25). Note that (3.25) and (3.6) give

$$
\sup _{0 \leq t \leq T} \sigma\|\nabla u\|_{L^{6}} \leq C(\bar{\rho}) C_{0}^{1 / 12} .
$$

This combining with (3.41) and (3.6) leads to

$$
\begin{aligned}
& \sup _{0<t \leq T} \sigma^{4}\left\|\nabla^{2} \theta\right\|_{L^{2}}^{2} \\
& \leq C(\bar{\rho}) \sup _{0<t \leq T} \sigma^{2}\left(\|\nabla \theta\|_{L^{2}}^{2}+\|\nabla u\|_{L^{2}}^{2}\right) \sup _{0<t \leq T} \sigma^{2}\left(\left\|\rho^{1 / 2} \dot{u}\right\|_{L^{2}}^{2}+\|\nabla \theta\|_{L^{2}}^{2}+1\right) \\
& \quad+C \sup _{0<t \leq T}\left(\sigma^{4}\|\rho \dot{\theta}\|_{L^{2}}^{2}+\left(\sigma\|\nabla u\|_{L^{2}}\right)\left(\sigma\|\nabla u\|_{L^{6}}\right)^{3}\right), \\
& \leq C(\bar{\rho}) C_{0}^{1 / 8}
\end{aligned}
$$

which together with (2.21), (2.22), and (3.6) yields that

$$
\begin{aligned}
\sup _{0<t \leq T} \sigma^{2}\|\theta-1\|_{L^{\infty}} & \leq \sup _{0<t \leq T} \sigma^{2}\left(\|\nabla \theta\|_{L^{2}}+\left\|\nabla^{2} \theta\right\|_{L^{2}}\right) \\
& \leq C(\bar{\rho}) C_{0}^{1 / 16} \leq 1 / 2
\end{aligned}
$$

provided $C_{0} \leq \varepsilon_{2}^{2} \triangleq \min \left\{1,(2 C(\bar{\rho}))^{-16}\right\}$. We assume that $C_{0} \leq \min \left\{\varepsilon_{2}^{1}, \varepsilon_{2}^{2}\right\}$. It follows from (3.45) that, for all $(x, t) \in \mathbb{R}^{3} \times[\sigma(T), T]$,

$$
1 / 2 \leq \theta(x, t) \leq 3 / 2,
$$

which as well as (2.20) and (3.13) $-(3.16)$ gives

$$
\begin{aligned}
& \sup _{\sigma(T) \leq t \leq T} \int\left(\rho|u|^{2}+(\rho-1)^{2}+\frac{R}{2(\gamma-1)} \rho(\theta-1)^{2}\right) d x \\
& +\int_{\sigma(T)}^{T}\left(\|\nabla u\|_{L^{2}}^{2}+\|\nabla \theta\|_{L^{2}}^{2}\right) d t \leq C(\bar{\rho}) C_{0} .
\end{aligned}
$$

Finally, the combination of (3.39) with (3.47) yields

$$
\begin{aligned}
& \sup _{0 \leq t \leq T} \int\left(\rho|u|^{2}+(\rho-1)^{2}+\frac{R}{2(\gamma-1)} \rho(\theta-1)^{2}\right) d x \\
& +\int_{0}^{T}\left(\|\nabla u\|_{L^{2}}^{2}+\|\nabla \theta\|_{L^{2}}^{2}\right) d t \\
& \leq \max \left\{C(\bar{\rho}) C_{0}, C(\bar{\rho}, M) C_{0}^{1 / 3}\right\} \leq C_{0}^{1 / 4}
\end{aligned}
$$

provided

$$
C_{0} \leq \varepsilon_{2} \triangleq \min \left\{\varepsilon_{2}^{1}, \varepsilon_{2}^{2},(C(\bar{\rho}))^{-4 / 3},(C(\bar{\rho}, M))^{-12}\right\}
$$


The proof of Lemma 3.3 is completed.

Next, to estimate $A_{3}(T)$, we first establish the following Lemmas 3.4 and 3.5 concerning some elementary estimates on $\dot{u}$ and $\dot{\theta}$ for the case that the density may contain vacuum states. This approach is motivated by the basic estimates on $\dot{u}$ and $\dot{\theta}$ developed by Hoff [10] when the density is strictly away from vacuum. The estimate of $A_{3}(T)$ will be postponed to Lemma 3.6 .

Lemma 3.4 In addition to the conditions of Proposition 3.1, assume that $C_{0} \leq 1$. Let $(\rho, u, \theta)$ be a smooth solution of (1.6) (1.4) (1.7) on $\mathbb{R}^{3} \times(0, T]$ satisfying (3.6) with $K$ as in Lemma 3.2. Then there exist positive constants $C$ and $C_{1}$ both depending only on $\mu, \lambda, \kappa, R, \gamma, \bar{\rho}$, and $M$ such that, for any $\beta \in(0,1]$, the following estimates hold

$$
\begin{aligned}
& \left(\sigma B_{1}\right)^{\prime}(t)+\frac{3}{2} \int \sigma \rho|\dot{u}|^{2} d x \\
& \leq C C_{0}^{1 / 4} \sigma^{\prime}+2 \beta \sigma^{2}\left\|\rho^{1 / 2} \dot{\theta}\right\|_{L^{2}}^{2}+C \beta^{-1}\left(\|\nabla \theta\|_{L^{2}}^{2}+\|\nabla u\|_{L^{2}}^{2}\right)+C \sigma^{2}\|\nabla u\|_{L^{4}}^{4},
\end{aligned}
$$

and

$$
\begin{aligned}
& \left(\sigma^{2} \int \rho|\dot{u}|^{2} d x\right)_{t}+\frac{3 \mu}{2} \int \sigma^{2}|\nabla \dot{u}|^{2} d x \\
& \leq 2 \sigma \int \rho|\dot{u}|^{2} d x+C_{1} \sigma^{2}\left\|\rho^{1 / 2} \dot{\theta}\right\|_{L^{2}}^{2}+C\left(\|\nabla \theta\|_{L^{2}}^{2}+\|\nabla u\|_{L^{2}}^{2}\right)+C \sigma^{2}\|\nabla u\|_{L^{4}}^{4},
\end{aligned}
$$

where

$$
B_{1}(t) \triangleq \mu\|\nabla u\|_{L^{2}}^{2}+(\lambda+\mu)\|\operatorname{div} u\|_{L^{2}}^{2}+2 R \int \operatorname{div} u(\rho \theta-1) d x
$$

Proof. Multiplying $(1.6)_{2}$ by $\sigma \dot{u}$ and integrating the resulting equality over $\mathbb{R}^{3}$ lead to

$$
\begin{aligned}
\int \sigma \rho|\dot{u}|^{2} d x & =\int(-\sigma \dot{u} \cdot \nabla P+\mu \sigma \triangle u \cdot \dot{u}+(\lambda+\mu) \sigma \nabla \operatorname{div} u \cdot \dot{u}) d x \\
& \triangleq \sum_{i=1}^{3} M_{i} .
\end{aligned}
$$


Using $(1.6)_{1}$, we get after integration by parts that, for any $\beta \in(0,1]$,

$$
\begin{aligned}
M_{1}= & -\int \sigma \dot{u} \cdot \nabla P d x \\
= & \int \sigma(P \operatorname{div} u)_{t} d x+\int \sigma\left(-P_{t} \operatorname{div} u+P \operatorname{div}(u \cdot \nabla u)\right) d x \\
= & \int \sigma(P \operatorname{div} u)_{t} d x-R \int \sigma \operatorname{div} u\left(\rho \dot{\theta}-\rho u \cdot \nabla \theta+\theta \rho_{t}\right) d x \\
& +\int \sigma P u \cdot \nabla \operatorname{div} u d x+\int \sigma P \partial_{i} u^{j} \partial_{j} u^{i} d x \\
= & \int \sigma(P \operatorname{div} u)_{t} d x+R \int \sigma \operatorname{div} u(\rho u \cdot \nabla \theta+\theta u \cdot \nabla \rho+\theta \rho \operatorname{div} u) d x \\
& +\int \sigma P u \cdot \nabla \operatorname{div} u d x-R \int \sigma \operatorname{div} u \rho \dot{\theta} d x+\int \sigma P \partial_{i} u^{j} \partial_{j} u^{i} d x \\
= & R\left(\int \sigma(\rho \theta-1) \operatorname{div} u d x\right)_{t}-R \sigma^{\prime} \int(\rho \theta-1) \operatorname{div} u d x-R \int \sigma \operatorname{div} u \rho \dot{\theta} d x \\
& +\int \sigma P \partial_{i} u^{j} \partial_{j} u^{i} d x \\
\leq & R\left(\int \sigma(\rho \theta-1) \operatorname{div} u d x\right)_{t}+C \sigma^{\prime}\|\nabla u\|_{L^{2}}\|\rho \theta-1\|_{L^{2}} \\
& +C(\bar{\rho}) \sigma\|\nabla u\|_{L^{2}}\left\|\rho^{1 / 2} \dot{\theta}\right\|_{L^{2}}+C(\bar{\rho}) \sigma \int \theta|\nabla u|^{2} d x \\
\leq & R\left(\int \sigma(\rho \theta-1) \operatorname{div} u d x\right)_{t}+C(\bar{\rho}) C_{0}^{1 / 4} \sigma^{\prime}+\beta \sigma^{2}\left\|\rho^{1 / 2} \dot{\theta}\right\|_{L^{2}}^{2} \\
& +C(\bar{\rho}) \delta\left\|\rho^{1 / 2} \dot{u}\right\|_{L^{2}}^{2}+C(\bar{\rho}, \delta, M) \beta^{-1}\left(\|\nabla u\|_{L^{2}}^{2}+\|\nabla \theta\|_{L^{2}}^{2}\right)
\end{aligned}
$$

where in the last inequality we have used (3.24) and the following simple fact:

$$
\begin{aligned}
& \int \theta|\nabla u|^{2} d x \\
& \leq \int|\theta-1 \| \nabla u|^{2} d x+\int|\nabla u|^{2} d x \\
& \leq C\|\theta-1\|_{L^{6}}\|\nabla u\|_{L^{2}}^{3 / 2}\|\nabla u\|_{L^{6}}^{1 / 2}+\|\nabla u\|_{L^{2}}^{2} \\
& \leq C\|\nabla \theta\|_{L^{2}}\|\nabla u\|_{L^{2}}^{3 / 2}\left(\|\rho \dot{u}\|_{L^{2}}+\|\nabla \theta\|_{L^{2}}+1\right)^{1 / 2}+\|\nabla u\|_{L^{2}}^{2} \\
& \leq \delta\left(\|\nabla \theta\|_{L^{2}}^{2}+\left\|\rho^{1 / 2} \dot{u}\right\|_{L^{2}}^{2}\right)+C(\bar{\rho}, \delta, M)\|\nabla u\|_{L^{2}}^{2},
\end{aligned}
$$


due to (2.21), (3.35), and (3.25). Integration by parts gives

$$
\begin{aligned}
M_{2}= & \int \mu \sigma \triangle u \cdot \dot{u} d x \\
= & -\frac{\mu}{2}\left(\sigma\|\nabla u\|_{L^{2}}^{2}\right)_{t}+\frac{\mu}{2} \sigma^{\prime}\|\nabla u\|_{L^{2}}^{2}-\mu \sigma \int \partial_{i} u^{j} \partial_{i}\left(u^{k} \partial_{k} u^{j}\right) d x \\
= & -\frac{\mu}{2}\left(\sigma\|\nabla u\|_{L^{2}}^{2}\right)_{t}+\frac{\mu}{2} \sigma^{\prime}\|\nabla u\|_{L^{2}}^{2}-\mu \sigma \int \partial_{i} u^{j} \partial_{i} u^{k} \partial_{k} u^{j} d x \\
& +\frac{\mu}{2} \sigma \int|\nabla u|^{2} \operatorname{div} u d x \\
\leq & -\frac{\mu}{2}\left(\sigma\|\nabla u\|_{L^{2}}^{2}\right)_{t}+C\|\nabla u\|_{L^{2}}^{2}+C \int \sigma|\nabla u|^{3} d x \\
\leq & -\frac{\mu}{2}\left(\sigma\|\nabla u\|_{L^{2}}^{2}\right)_{t}+C\|\nabla u\|_{L^{2}}^{2}+C \sigma^{2}\|\nabla u\|_{L^{4}}^{4} .
\end{aligned}
$$

Similar to (3.54), we have

$$
\begin{aligned}
M_{3}= & -\frac{\lambda+\mu}{2}\left(\sigma\|\operatorname{div} u\|_{L^{2}}^{2}\right)_{t}+\frac{\lambda+\mu}{2} \sigma^{\prime}\|\operatorname{div} u\|_{L^{2}}^{2} \\
& -(\lambda+\mu) \sigma \int \operatorname{div} u \operatorname{div}(u \cdot \nabla u) d x \\
\leq & -\frac{\lambda+\mu}{2}\left(\sigma\|\operatorname{div} u\|_{L^{2}}^{2}\right)_{t}+C\|\nabla u\|_{L^{2}}^{2}+C \sigma^{2}\|\nabla u\|_{L^{4}}^{4} .
\end{aligned}
$$

Substituting (3.52), (3.54), and (3.55) into (3.51), we obtain (3.48) after choosing $\delta$ suitably small.

It remains to prove (3.49). For $m \geq 0$, operating $\sigma^{m} \dot{u}^{j}[\partial / \partial t+\operatorname{div}(u \cdot)]$ to $(1.6)_{2}^{j}$ and integrating the resulting equality over $\mathbb{R}^{3}$, we obtain after integration by parts that

$$
\begin{aligned}
& \left(\frac{\sigma^{m}}{2} \int \rho|\dot{u}|^{2} d x\right)_{t}-\frac{m}{2} \sigma^{m-1} \sigma^{\prime} \int \rho|\dot{u}|^{2} d x \\
& =-\int \sigma^{m} \dot{u}^{j}\left[\partial_{j} P_{t}+\operatorname{div}\left(\partial_{j} P u\right)\right] d x+\mu \int \sigma^{m} \dot{u}^{j}\left[\triangle u_{t}^{j}+\operatorname{div}\left(u \triangle u^{j}\right)\right] d x \\
& \quad+(\lambda+\mu) \int \sigma^{m} \dot{u}^{j}\left[\partial_{t} \partial_{j} \operatorname{div} u+\operatorname{div}\left(u \partial_{j} \operatorname{div} u\right)\right] d x \\
& \triangleq \sum_{i=1}^{3} N_{i} .
\end{aligned}
$$


We get after integration by parts and using the equation (1.6) $)_{1}$ that

$$
\begin{aligned}
N_{1}= & -\int \sigma^{m} \dot{u}^{j}\left[\partial_{j} P_{t}+\operatorname{div}\left(\partial_{j} P u\right)\right] d x \\
= & R \int \sigma^{m} \partial_{j} \dot{u}^{j}(\rho \dot{\theta}-\rho u \cdot \nabla \theta-\theta u \cdot \nabla \rho-\theta \rho \operatorname{div} u) d x \\
& +\int \sigma^{m} \partial_{k} \dot{u}^{j} \partial_{j} P u^{k} d x \\
= & R \int \sigma^{m} \partial_{j} \dot{u}^{j}(\rho \dot{\theta}-\theta \rho \operatorname{div} u) d x+\int \sigma^{m} P \operatorname{div} \dot{u} \operatorname{div} u d x \\
& -\int \sigma^{m} P \partial_{k} \dot{u}^{j} \partial_{j} u^{k} d x \\
\leq & \frac{\mu}{8} \int \sigma^{m}|\nabla \dot{u}|^{2} d x+C(\bar{\rho}) \sigma^{m}\left(\|\rho \dot{\theta}\|_{L^{2}}^{2}+\int \theta^{2}|\nabla u|^{2} d x\right) \\
\leq & \frac{\mu}{8} \int \sigma^{m}|\nabla \dot{u}|^{2} d x+C(\bar{\rho}) \sigma^{m}\left\|\rho^{1 / 2} \dot{\theta}\right\|_{L^{2}}^{2} \\
& +C(\bar{\rho}, M)\left(\|\nabla u\|_{L^{2}}^{2}+\|\nabla \theta\|_{L^{2}}^{2}\right)\left(\sigma^{m}\left\|\rho^{1 / 2} \dot{u}\right\|_{L^{2}}^{2}+\sigma^{m}\|\nabla \theta\|_{L^{2}}^{2}+1\right)
\end{aligned}
$$

where in the last inequality we have used (3.42). Integration by parts leads to

$$
\begin{aligned}
N_{2} & =\mu \int \sigma^{m} \dot{u}^{j}\left[\triangle u_{t}^{j}+\operatorname{div}\left(u \triangle u^{j}\right)\right] d x \\
& =-\mu \int \sigma^{m}\left(\partial_{i} \dot{u}^{j} \partial_{i} u_{t}^{j}+\triangle u^{j} u \cdot \nabla \dot{u}^{j}\right) d x \\
& =-\mu \int \sigma^{m}\left(|\nabla \dot{u}|^{2}-\partial_{i} \dot{u}^{j} u^{k} \partial_{k} \partial_{i} u^{j}-\partial_{i} \dot{u}^{j} \partial_{i} u^{k} \partial_{k} u^{j}+\triangle u^{j} u \cdot \nabla \dot{u}^{j}\right) d x \\
& =-\mu \int \sigma^{m}\left(|\nabla \dot{u}|^{2}+\partial_{i} \dot{u}^{j} \partial_{i} u^{j} \operatorname{div} u-\partial_{i} \dot{u}^{j} \partial_{i} u^{k} \partial_{k} u^{j}-\partial_{i} u^{j} \partial_{i} u^{k} \partial_{k} \dot{u}^{j}\right) d x \\
& \leq-\frac{7 \mu}{8} \int \sigma^{m}|\nabla \dot{u}|^{2} d x+C \int \sigma^{m}|\nabla u|^{4} d x .
\end{aligned}
$$

Similarly, we have

$$
\begin{aligned}
N_{3} & \leq-\frac{7(\mu+\lambda)}{8} \int \sigma^{m}(\operatorname{div} \dot{u})^{2} d x+C \int \sigma^{m}|\nabla u|^{4} d x \\
& \leq C \int \sigma^{m}|\nabla u|^{4} d x
\end{aligned}
$$

where in the second inequality we have used $\mu+\lambda \geq 0$ due to (1.2).

Substituting (3.57)-(3.59) into (3.56) yields that there exists some $C_{1}$ depending only on $\mu, \lambda, \kappa, R, \gamma, \bar{\rho}$, and $M$ such that

$$
\begin{aligned}
& \left(\sigma^{m} \int \rho|\dot{u}|^{2} d x\right)_{t}+\frac{3 \mu}{2} \int \sigma^{m}|\nabla \dot{u}|^{2} d x \\
& \leq m \sigma^{m-1} \sigma^{\prime} \int \rho|\dot{u}|^{2} d x+C_{1} \sigma^{m}\left\|\rho^{1 / 2} \dot{\theta}\right\|_{L^{2}}^{2}+C \sigma^{m}\|\nabla u\|_{L^{4}}^{4} \\
& \quad+C(\bar{\rho}, M)\left(\|\nabla \theta\|_{L^{2}}^{2}+\|\nabla u\|_{L^{2}}^{2}\right)\left(\sigma^{m}\left\|\rho^{1 / 2} \dot{u}\right\|_{L^{2}}^{2}+\sigma^{m}\|\nabla \theta\|_{L^{2}}^{2}+1\right) .
\end{aligned}
$$

Taking $m=2$ in (3.60) together with (3.6) gives (3.49) directly. We thus finish the proof of Lemma 3.4 . 
Lemma 3.5 In addition to the conditions of Proposition [3.1, assume that $C_{0} \leq 1$. Let $(\rho, u, \theta)$ be a smooth solution of (1.6) (1.4) (1.7) on $\mathbb{R}^{3} \times(0, T]$ satisfying (3.6) with $K$ as in Lemma 3.2. Then there exists a positive constant $C$ depending only on $\mu, \lambda, \kappa, R, \gamma, \bar{\rho}$, and $M$ such that the following estimate holds

$$
\begin{aligned}
& \left(\sigma^{2} \varphi\right)^{\prime}(t)+\sigma^{2} \int\left(\mu|\nabla \dot{u}|^{2}+\rho(\dot{\theta})^{2}\right) d x \\
& \leq C\left(\|\nabla u\|_{L^{2}}^{2}+\|\nabla \theta\|_{L^{2}}^{2}\right)+2 \sigma \int \rho|\dot{u}|^{2} d x+C \sigma^{2}\|\nabla u\|_{L^{4}}^{4},
\end{aligned}
$$

where $\varphi(t)$ is defined by

$$
\varphi(t) \triangleq \int \rho|\dot{u}|^{2}(x, t) d x+\left(C_{1}+1\right) B_{2}(t)
$$

with $C_{1}$ as in Lemma 3.4 and

$$
B_{2}(t) \triangleq \frac{\gamma-1}{R}\left(\kappa\|\nabla \theta\|_{L^{2}}^{2}-2 \lambda \int(\operatorname{div} u)^{2} \theta d x-4 \mu \int|\mathfrak{D}(u)|^{2} \theta d x\right) .
$$

Proof. For $m \geq 0$, multiplying (1.6) 3 by $\sigma^{m} \dot{\theta}$ and integrating the resulting equality over $\mathbb{R}^{3}$ yield that

$$
\begin{aligned}
& \frac{R \sigma^{m}}{\gamma-1} \int \rho|\dot{\theta}|^{2} d x+\frac{\kappa \sigma^{m}}{2}\left(\|\nabla \theta\|_{L^{2}}^{2}\right)_{t} \\
& =-\kappa \sigma^{m} \int \nabla \theta \cdot \nabla(u \cdot \nabla \theta) d x+\lambda \sigma^{m} \int(\operatorname{div} u)^{2} \dot{\theta} d x \\
& \quad+2 \mu \sigma^{m} \int|\mathfrak{D}(u)|^{2} \dot{\theta} d x-R \sigma^{m} \int \rho \theta \operatorname{div} u \dot{\theta} d x \\
& \triangleq \sum_{i=1}^{4} I_{i} .
\end{aligned}
$$

First, it follows from (2.21) and (3.6) that

$$
\begin{aligned}
\left|I_{1}\right| \leq & C \sigma^{m} \int|\nabla u \| \nabla \theta|^{2} d x \\
\leq & C \sigma^{m}\|\nabla u\|_{L^{2}}\|\nabla \theta\|_{L^{2}}^{1 / 2}\|\nabla \theta\|_{L^{6}}^{3 / 2} \\
\leq & \delta \sigma^{m}\left\|\nabla^{2} \theta\right\|_{L^{2}}^{2}+C(\bar{\rho}, \delta, M) \sigma^{m}\|\nabla \theta\|_{L^{2}}^{2} \\
\leq & C(\bar{\rho}, \delta, M)\left(\|\nabla \theta\|_{L^{2}}^{2}+\|\nabla u\|_{L^{2}}^{2}\right)\left(\sigma^{m}\left\|\rho^{1 / 2} \dot{u}\right\|_{L^{2}}^{2}+\sigma^{m}\|\nabla \theta\|_{L^{2}}^{2}+1\right) \\
& +C(\bar{\rho}) \delta \sigma^{m}\left\|\rho^{1 / 2} \dot{\theta}\right\|_{L^{2}}^{2}+C \delta \sigma^{m}\|\nabla u\|_{L^{4}}^{4},
\end{aligned}
$$

where in the last inequality we have used (3.41). 
Next, integration by parts yields that, for any $\eta \in(0,1]$,

$$
\begin{aligned}
I_{2}= & \lambda \sigma^{m} \int(\operatorname{div} u)^{2} \theta_{t} d x+\lambda \sigma^{m} \int(\operatorname{div} u)^{2} u \cdot \nabla \theta d x \\
= & \lambda \sigma^{m}\left(\int(\operatorname{div} u)^{2} \theta d x\right)_{t}-2 \lambda \sigma^{m} \int \theta \operatorname{div} u \operatorname{div}(\dot{u}-u \cdot \nabla u) d x \\
& +\lambda \sigma^{m} \int(\operatorname{div} u)^{2} u \cdot \nabla \theta d x \\
= & \lambda \sigma^{m}\left(\int(\operatorname{div} u)^{2} \theta d x\right)_{t}-2 \lambda \sigma^{m} \int \theta \operatorname{div} u \operatorname{div} \dot{u} d x \\
& +2 \lambda \sigma^{m} \int \theta \operatorname{div} u \operatorname{div}(u \cdot \nabla u) d x+\lambda \sigma^{m} \int(\operatorname{div} u)^{2} u \cdot \nabla \theta d x \\
= & \lambda \sigma^{m}\left(\int(\operatorname{div} u)^{2} \theta d x\right)_{t}-2 \lambda \sigma^{m} \int \theta \operatorname{div} u \operatorname{div} \dot{u} d x \\
& +2 \lambda \sigma^{m} \int \theta \operatorname{div} u \partial_{i} u^{j} \partial_{j} u^{i} d x+\lambda \sigma^{m} \int u \cdot \nabla\left(\theta(\operatorname{div} u)^{2}\right) d x \\
\leq & \lambda\left(\sigma^{m} \int(\operatorname{div} u)^{2} \theta d x\right)_{t}-\lambda m \sigma^{m-1} \sigma^{\prime} \int(\operatorname{div} u)^{2} \theta d x \\
& +\eta \sigma^{m}\|\nabla \dot{u}\|_{L^{2}}^{2}+C \eta^{-1} \sigma^{m} \int \theta^{2}|\nabla u|^{2} d x+\sigma^{m}\|\nabla u\|_{L^{4}}^{4} \\
\leq & \lambda\left(\sigma^{m} \int(\operatorname{div} u)^{2} \theta d x\right)_{t}-\lambda m \sigma^{m-1} \sigma^{\prime} \int(\operatorname{div} u)^{2} \theta d x \\
& +C(\bar{\rho}) \eta^{-1}\left(\|\nabla u\|_{L^{2}}^{2}+\|\nabla \theta\|_{L^{2}}^{2}\right)\left(\sigma^{m}\left\|\rho^{1 / 2} \dot{u}\right\|_{L^{2}}^{2}+\sigma^{m}\|\nabla \theta\|_{L^{2}}^{2}+1\right) \\
& +\eta \sigma^{m}\|\nabla \dot{u}\|_{L^{2}}^{2}+\sigma^{m}\|\nabla u\|_{L^{4}}^{4},
\end{aligned}
$$

where in the last inequality we have used (3.42).

Then, similar to (3.66), we have that, for any $\eta \in(0,1]$,

$$
\begin{aligned}
I_{3} \leq & 2 \mu\left(\sigma^{m} \int|\mathfrak{D}(u)|^{2} \theta d x\right)_{t}-2 \mu m \sigma^{m-1} \sigma^{\prime} \int|\mathfrak{D}(u)|^{2} \theta d x \\
& +C(\bar{\rho}) \eta^{-1}\left(\|\nabla u\|_{L^{2}}^{2}+\|\nabla \theta\|_{L^{2}}^{2}\right)\left(\sigma^{m}\left\|\rho^{1 / 2} \dot{u}\right\|_{L^{2}}^{2}+\sigma^{m}\|\nabla \theta\|_{L^{2}}^{2}+1\right) \\
& +C \eta \sigma^{m}\|\nabla \dot{u}\|_{L^{2}}^{2}+C \sigma^{m}\|\nabla u\|_{L^{4}}^{4} .
\end{aligned}
$$

Finally, it follows from (3.42) that

$$
\begin{aligned}
\left|I_{4}\right| \leq & C(\bar{\rho}) \sigma^{m} \int \theta^{2}|\nabla u|^{2} d x+\frac{R}{4(\gamma-1)} \sigma^{m} \int \rho|\dot{\theta}|^{2} d x \\
\leq & C(\bar{\rho})\left(\|\nabla \theta\|_{L^{2}}^{2}+\|\nabla u\|_{L^{2}}^{2}\right)\left(\sigma^{m}\left\|\rho^{1 / 2} \dot{u}\right\|_{L^{2}}^{2}+\sigma^{m}\|\nabla \theta\|_{L^{2}}^{2}+1\right) \\
& +\frac{R}{4(\gamma-1)} \sigma^{m} \int \rho|\dot{\theta}|^{2} d x .
\end{aligned}
$$

Substituting (3.65)-(3.68) into (3.64), we obtain after using (2.20), (3.11), and choosing $\delta$ suitably small that, for any $\eta \in(0,1]$,

$$
\begin{aligned}
& \left(\sigma^{m} B_{2}\right)^{\prime}(t)+\sigma^{m} \int \rho(\dot{\theta})^{2} d x \\
& \leq C(\bar{\rho}, M) \eta^{-1}\left(\|\nabla u\|_{L^{2}}^{2}+\|\nabla \theta\|_{L^{2}}^{2}\right)\left(\sigma^{m}\left\|\rho^{1 / 2} \dot{u}\right\|_{L^{2}}^{2}+\sigma^{m}\|\nabla \theta\|_{L^{2}}^{2}+1\right) \\
& +C \eta \sigma^{m}\|\nabla \dot{u}\|_{L^{2}}^{2}+C m \sigma^{m-1} \sigma^{\prime}\|\nabla \theta\|_{L^{2}}^{2}+C \sigma^{m}\|\nabla u\|_{L^{4}}^{4},
\end{aligned}
$$


with $B_{2}$ as in (3.63). For $C_{1}$ as in Lemma 3.4 (see also (3.60)), adding (3.69) multiplied by $C_{1}+1$ to (3.60), we obtain after choosing $\eta$ suitably small that, for $\varphi$ as in (3.62) and for $m \geq 0$,

$$
\begin{aligned}
& \left(\sigma^{m} \varphi\right)^{\prime}(t)+\sigma^{m} \int\left(\mu|\nabla \dot{u}|^{2}+\rho(\dot{\theta})^{2}\right) d x \\
& \leq C(\bar{\rho}, M)\left(\|\nabla u\|_{L^{2}}^{2}+\|\nabla \theta\|_{L^{2}}^{2}\right)\left(\sigma^{m}\left\|\rho^{1 / 2} \dot{u}\right\|_{L^{2}}^{2}+\sigma^{m}\|\nabla \theta\|_{L^{2}}^{2}+1\right) \\
& \quad+m \sigma^{\prime} \sigma^{m-1} \int \rho|\dot{u}|^{2} d x+C(\bar{\rho}, M) m \sigma^{m-1} \sigma^{\prime}\|\nabla \theta\|_{L^{2}}^{2} \\
& \quad+C(\bar{\rho}, M) \sigma^{m}\|\nabla u\|_{L^{4}}^{4} .
\end{aligned}
$$

Taking $m=2$ in (3.70) together with (3.6) gives (3.61). The proof of Lemma 3.5 is completed.

Next, we will use Lemmas 3.4 and 3.5 to obtain the following estimate on $A_{3}(T)$.

Lemma 3.6 Under the conditions of Proposition 3.1, there exists a positive constant $\varepsilon_{3}$ depending only on $\mu, \lambda, \kappa, R, \gamma, \bar{\rho}$, and $M$ such that if $(\rho, u, \theta)$ is a smooth solution of (1.6) (1.4) [1.7) on $\mathbb{R}^{3} \times(0, T]$ satisfying (3.6) with $K$ as in Lemma 3.2, the following estimate holds

$$
A_{3}(T) \leq C_{0}^{1 / 6},
$$

provided $C_{0} \leq \varepsilon_{3}$.

Proof. First, assume that $C_{0} \leq 1$. It follows from (2.26), (2.28), (2.21), (3.35), and (3.6) that

$$
\begin{aligned}
\|\nabla u\|_{L^{4}}^{4} & \leq C\|G\|_{L^{4}}^{4}+C\|\omega\|_{L^{4}}^{4}+C\|\rho \theta-1\|_{L^{4}}^{4} \\
& \leq C(\bar{\rho})\left(\|\nabla u\|_{L^{2}}+1\right)\left\|\rho^{1 / 2} \dot{u}\right\|_{L^{2}}^{3}+C\|\rho(\theta-1)\|_{L^{4}}^{4}+C\|\rho-1\|_{L^{4}}^{4} \\
& \leq C(\bar{\rho}, M)\left\|\rho^{1 / 2} \dot{u}\right\|_{L^{2}}^{3}+C(\bar{\rho})\|\rho(\theta-1)\|_{L^{2}}\|\nabla \theta\|_{L^{2}}^{3}+C\|\rho-1\|_{L^{4}}^{4} \\
& \leq C(\bar{\rho}, M)\left\|\rho^{1 / 2} \dot{u}\right\|_{L^{2}}^{3}+C(\bar{\rho})\|\nabla \theta\|_{L^{2}}^{3}+C\|\rho-1\|_{L^{4}}^{4}
\end{aligned}
$$

which together with (3.6) yields

$$
\sigma\|\nabla u\|_{L^{4}}^{4} \leq C(\bar{\rho}, M) C_{0}^{1 / 12}\left\|\rho^{1 / 2} \dot{u}\right\|_{L^{2}}^{2}+C(\bar{\rho})\|\nabla \theta\|_{L^{2}}^{2}+C \sigma\|\rho-1\|_{L^{4}}^{4} .
$$

This fact combining with (3.61) gives that, for $\varphi(t)$ as in (3.62),

$$
\begin{aligned}
& \left(\sigma^{2} \varphi\right)^{\prime}(t)+\sigma^{2} \int\left(\mu|\nabla \dot{u}|^{2}+\rho(\dot{\theta})^{2}\right) d x \\
& \leq C(\bar{\rho}, M)\left(\|\nabla u\|_{L^{2}}^{2}+\|\nabla \theta\|_{L^{2}}^{2}\right)+\left(C(\bar{\rho}, M) C_{0}^{1 / 12}+2\right) \sigma \int \rho|\dot{u}|^{2} d x \\
& \quad+C(\bar{\rho}, M) \sigma^{2}\|\rho-1\|_{L^{4}}^{4} \\
& \leq C(\bar{\rho}, M)\left(\|\nabla u\|_{L^{2}}^{2}+\|\nabla \theta\|_{L^{2}}^{2}\right)+3 \sigma \int \rho|\dot{u}|^{2} d x+C(\bar{\rho}, M) \sigma^{2}\|\rho-1\|_{L^{4}}^{4},
\end{aligned}
$$

provided $C_{0} \leq \varepsilon_{3}^{1} \triangleq \min \left\{1,(C(\bar{\rho}, M))^{-12}\right\}$ 
Next, to estimate the second term on the righthand side of (3.74), we substitute (3.73) into (3.48) to obtain that, for $B_{1}(t)$ as in (3.50),

$$
\begin{aligned}
& \left(\sigma B_{1}\right)^{\prime}(t)+\int \sigma \rho|\dot{u}|^{2} d x \\
& \leq C(\bar{\rho}, M) C_{0}^{1 / 4} \sigma^{\prime}+2 \beta \sigma^{2}\left\|\rho^{1 / 2} \dot{\theta}\right\|_{L^{2}}^{2}+C(\bar{\rho}, M) \beta^{-1}\left(\|\nabla \theta\|_{L^{2}}^{2}+\|\nabla u\|_{L^{2}}^{2}\right) \\
& \quad+C(\bar{\rho}, M) \sigma^{2}\|\rho-1\|_{L^{4}}^{4},
\end{aligned}
$$

provided $C_{0} \leq \varepsilon_{3}^{2} \triangleq \min \left\{1,(2 C(\bar{\rho}, M))^{-12}\right\}$. From now on, we assume that $C_{0} \leq$ $\min \left\{\varepsilon_{3}^{1}, \varepsilon_{3}^{2}\right\}$. It follows from (3.62), (3.63), and (3.53) that

$$
\varphi(t) \geq \frac{1}{2} \int \rho|\dot{u}|^{2} d x+\frac{\kappa(\gamma-1)}{2 R}\|\nabla \theta\|_{L^{2}}^{2}-C_{2}(\bar{\rho}, M)\|\nabla u\|_{L^{2}}^{2},
$$

which together with (3.35) directly gives

$$
\int \rho|\dot{u}|^{2}(x, t) d x+\|\nabla \theta(\cdot, t)\|_{L^{2}}^{2} \leq 2\left(\frac{R}{\kappa(\gamma-1)}+1\right) \varphi(t)+C(\bar{\rho}, M) .
$$

For $C_{2}$ as in (3.76), adding (3.75) multiplied by $2\left(C_{2}+2 \mu+1\right) / \mu$ to (3.74), we obtain after choosing $\beta$ suitably small that

$$
\begin{aligned}
& B_{3}^{\prime}(t)+\frac{1}{2} \int\left(\sigma \rho|\dot{u}|^{2}+\mu \sigma^{2}|\nabla \dot{u}|^{2}+\sigma^{2} \rho(\dot{\theta})^{2}\right) d x \\
& \leq C(\bar{\rho}, M) C_{0}^{1 / 4} \sigma^{\prime}+C(\bar{\rho}, M)\left(\|\nabla \theta\|_{L^{2}}^{2}+\|\nabla u\|_{L^{2}}^{2}\right)+C(\bar{\rho}, M) \sigma^{2}\|\rho-1\|_{L^{4}}^{4},
\end{aligned}
$$

where

$$
B_{3}(t) \triangleq \sigma^{2} \varphi+\frac{2\left(C_{2}+2 \mu+1\right)}{\mu} \sigma B_{1} .
$$

Note that (3.50) and (3.24) lead to

$$
\begin{aligned}
B_{1}(t) & \geq \mu\|\nabla u\|_{L^{2}}^{2}+(\lambda+\mu)\|\operatorname{div} u\|_{L^{2}}^{2}-\left(\frac{\mu}{2}\|\nabla u\|_{L^{2}}^{2}+C(\bar{\rho})\|\rho \theta-1\|_{L^{2}}^{2}\right) \\
& \geq \frac{\mu}{2}\|\nabla u\|_{L^{2}}^{2}-C(\bar{\rho}) C_{0}^{1 / 4},
\end{aligned}
$$

which together with $(\underline{3.76})$ and $(3.79)$ gives

$$
B_{3}(t) \geq \frac{\sigma^{2}}{2} \int \rho|\dot{u}|^{2} d x+\frac{\kappa(\gamma-1)}{2 R} \sigma^{2}\|\nabla \theta\|_{L^{2}}^{2}+\sigma\|\nabla u\|_{L^{2}}^{2}-C(\bar{\rho}, M) C_{0}^{1 / 4} .
$$

We claim that

$$
\int_{0}^{T} \sigma^{2}\|\rho-1\|_{L^{4}}^{4} d t \leq C(\bar{\rho}, M) C_{0}^{1 / 4},
$$

which combining with (3.78), (3.81), and (3.6) yields

$$
A_{3}(T) \leq C(\bar{\rho}, M) C_{0}^{1 / 4} \leq C_{0}^{1 / 6}
$$

provided $C_{0} \leq \varepsilon_{3} \triangleq \min \left\{\varepsilon_{3}^{1}, \varepsilon_{3}^{2},(C(\bar{\rho}, M))^{-12}\right\}$.

Finally, it remains to prove (3.82). In fact, it follows from (1.6) 1 and (3.23) that $\rho-1$ satisfies

$$
\begin{aligned}
& (\rho-1)_{t}+\frac{R}{2 \mu+\lambda}(\rho-1) \\
& =-u \cdot \nabla(\rho-1)-(\rho-1) \operatorname{div} u-\frac{G}{2 \mu+\lambda}-\frac{R \rho(\theta-1)}{2 \mu+\lambda} .
\end{aligned}
$$


Multiplying (3.84) by $4(\rho-1)^{3}$ and integrating the resulting equality over $\mathbb{R}^{3}$, we obtain that

$$
\begin{aligned}
\left(\|\rho-1\|_{L^{4}}^{4}\right)_{t}+\frac{4 R}{2 \mu+\lambda}\|\rho-1\|_{L^{4}}^{4} & -3 \int(\rho-1)^{4} \operatorname{div} u d x-\frac{4}{2 \mu+\lambda} \int(\rho-1)^{3} G d x \\
= & -\frac{4 R}{2 \mu+\lambda} \int(\rho-1)^{3} \rho(\theta-1) d x \\
\leq & \frac{2 R}{2 \mu+\lambda}\|\rho-1\|_{L^{4}}^{4}+C(\bar{\rho})\|\nabla u\|_{L^{2}}^{2}+C\|\rho-1\|_{L^{4}}^{3}\|G\|_{L^{2}}^{1 / 4}\|\nabla G\|_{L^{2}}^{3 / 4} \\
& +C(\bar{\rho})\|\rho-1\|_{L^{4}}^{3}\|\rho(\theta-1)\|_{L^{2}}^{1 / 4}\|\nabla \theta\|_{L^{2}}^{3 / 4} \\
\leq & \frac{3 R}{2 \mu+\lambda}\|\rho-1\|_{L^{4}}^{4}+C(\bar{\rho})\|\nabla u\|_{L^{2}}^{2}+C(\bar{\rho}, M)\left(\left\|\rho^{1 / 2} \dot{u}\right\|_{L^{2}}^{3}+\|\nabla \theta\|_{L^{2}}^{3}\right)
\end{aligned}
$$

where in the last inequality, we have used (3.6), (3.35), (3.24), (2.27), and (3.9). It thus follows from (3.85) that

$$
\begin{aligned}
& \left(\|\rho-1\|_{L^{4}}^{4}\right)_{t}+\frac{R}{2 \mu+\lambda}\|\rho-1\|_{L^{4}}^{4} \\
& \leq C(\bar{\rho}, M)\left(\left\|\rho^{1 / 2} \dot{u}\right\|_{L^{2}}^{3}+\|\nabla \theta\|_{L^{2}}^{3}\right)+C(\bar{\rho})\|\nabla u\|_{L^{2}}^{2} .
\end{aligned}
$$

Multiplying (3.86) by $\sigma^{n}$ with $n \geq 1$, integrating the resulting inequality over $(0, T)$, we obtain by using (3.9) and (3.6) that

$$
\begin{aligned}
& \int_{0}^{T} \sigma^{n}\|\rho-1\|_{L^{4}}^{4} d t \\
& \leq C(\bar{\rho}, M) A_{3}^{1 / 2}(T) \int_{0}^{T} \sigma^{n-1}\left(\left\|\rho^{1 / 2} \dot{u}\right\|_{L^{2}}^{2}+\|\nabla \theta\|_{L^{2}}^{2}\right) d t \\
& \quad+C(\bar{\rho}) C_{0}^{1 / 4}+C \int_{0}^{\sigma(T)}\|\rho-1\|_{L^{4}}^{4} d t \\
& \leq C(\bar{\rho}, M) C_{0}^{1 / 4}+C(\bar{\rho}, M) C_{0}^{1 / 12} \int_{0}^{T} \sigma^{n-1}\left\|\rho^{1 / 2} \dot{u}\right\|_{L^{2}}^{2} d t
\end{aligned}
$$

which together with (3.6) directly gives (3.82). We thus complete the proof of Lemma 3.6.

We now proceed to derive a uniform (in time) upper bound for the density, which turns out to be the key to obtain all the higher order estimates and thus to extend the classical solution globally.

Lemma 3.7 Under the conditions of Proposition 3.1, there exists a positive constant $\varepsilon_{4}$ depending only on $\mu, \lambda, \kappa, R, \gamma, \bar{\rho}$, and $M$ such that if $(\rho, u, \theta)$ is a smooth solution of (1.6) (1.4) (1.7) on $\mathbb{R}^{3} \times(0, T]$ satisfying (3.6) with $K$ as in Lemma 3.2, the following estimate holds

$$
\sup _{0 \leq t \leq T}\|\rho(\cdot, t)\|_{L^{\infty}} \leq \frac{3 \bar{\rho}}{2}
$$

provided $C_{0} \leq \varepsilon_{4}$ 
Proof. First, assume $C_{0} \leq 1$. Taking $n=1$ in (3.87) as well as (3.6) yields

$$
\int_{0}^{T} \sigma\|\rho-1\|_{L^{4}}^{4} d t \leq C(\bar{\rho}, M) .
$$

Choosing $m=1$ in (3.70) together with (3.77) and (3.73) yields that, for $\varphi(t)$ as in (3.62),

$$
\begin{aligned}
& (\sigma \varphi)^{\prime}(t)+\sigma \int\left(\mu|\nabla \dot{u}|^{2}+\rho(\dot{\theta})^{2}\right) d x \\
& \leq C(\bar{\rho}, M)\left(\|\nabla u\|_{L^{2}}^{2}+\|\nabla \theta\|_{L^{2}}^{2}\right)(\sigma \varphi)+C(\bar{\rho}, M)\left(\|\nabla u\|_{L^{2}}^{2}+\|\nabla \theta\|_{L^{2}}^{2}\right) \\
& \quad+C(\bar{\rho}, M) \int \rho|\dot{u}|^{2} d x+C(\bar{\rho}, M) \sigma\|\rho-1\|_{L^{4}}^{4},
\end{aligned}
$$

which combining with (3.6), (3.89), and Gronwall's inequality yields that

$$
\sup _{0 \leq t \leq \sigma(T)} \sigma \varphi(t)+\int_{0}^{\sigma(T)} \sigma \int\left(\mu|\nabla \dot{u}|^{2}+\rho(\dot{\theta})^{2}\right) d x d t \leq C(\bar{\rho}, M) .
$$

The combination of (3.77) with (3.90) thus directly gives

$$
\begin{aligned}
& \sup _{0 \leq t \leq \sigma(T)} \sigma\left(\int \rho|\dot{u}|^{2} d x+\|\nabla \theta\|_{L^{2}}^{2}\right) \\
& +\int_{0}^{\sigma(T)} \sigma \int\left(|\nabla \dot{u}|^{2}+\rho(\dot{\theta})^{2}\right) d x d t \leq C(\bar{\rho}, M) .
\end{aligned}
$$

Next, it follows from (3.41), (3.91), (3.73), (3.6), and (3.89) that

$$
\begin{aligned}
& \int_{0}^{T} \sigma\left\|\nabla^{2} \theta\right\|_{L^{2}}^{2} d t \\
& \leq C(\bar{\rho}, M) \int_{0}^{T}\left(\sigma\|\rho \dot{\theta}\|_{L^{2}}^{2}+\left\|\rho^{1 / 2} \dot{u}\right\|_{L^{2}}^{2}+\|\nabla u\|_{L^{2}}^{2}+\|\nabla \theta\|_{L^{2}}^{2}+\sigma\|\rho-1\|_{L^{4}}^{4}\right) d t \\
& \leq C(\bar{\rho}, M)
\end{aligned}
$$

which together with (2.22), (2.21), and (3.6) gives

$$
\begin{aligned}
& \int_{0}^{\sigma(T)}\|\theta-1\|_{L^{\infty}} d t \\
& \leq C \int_{0}^{\sigma(T)}\|\theta-1\|_{L^{6}}^{1 / 2}\|\nabla \theta\|_{L^{6}}^{1 / 2} d t \\
& \leq C \int_{0}^{\sigma(T)}\|\nabla \theta\|_{L^{2}}^{1 / 2}\left(\sigma\left\|\nabla^{2} \theta\right\|_{L^{2}}^{2}{ }^{1 / 4} \sigma^{-1 / 4} d t\right. \\
& \leq C\left(\int_{0}^{\sigma(T)}\|\nabla \theta\|_{L^{2}}^{2} d t\right)^{1 / 4}\left(\int_{0}^{\sigma(T)} \sigma\left\|\nabla^{2} \theta\right\|_{L^{2}}^{2} d t\right)^{1 / 4}\left(\int_{0}^{\sigma(T)} \sigma^{-1 / 2} d t\right)^{1 / 2} \\
& \leq C(\bar{\rho}, M) C_{0}^{1 / 16},
\end{aligned}
$$


and

$$
\begin{aligned}
& \int_{\sigma(T)}^{T}\|\theta-1\|_{L^{\infty}}^{2} d t \\
& \leq C \int_{\sigma(T)}^{T}\|\nabla \theta\|_{L^{2}}\left\|\nabla^{2} \theta\right\|_{L^{2}} d t \\
& \leq C\left(\int_{\sigma(T)}^{T}\|\nabla \theta\|_{L^{2}}^{2} d t\right)^{1 / 2}\left(\int_{\sigma(T)}^{T}\left\|\nabla^{2} \theta\right\|_{L^{2}}^{2} d t\right)^{1 / 2} \\
& \leq C(\bar{\rho}, M) C_{0}^{1 / 8} .
\end{aligned}
$$

Next, (2.22), (2.27), (3.91), and (3.6) lead to

$$
\begin{aligned}
& \int_{0}^{\sigma(T)}\|G\|_{L^{\infty}} d t \\
& \leq C \int_{0}^{\sigma(T)}\|\nabla G\|_{L^{2}}^{1 / 2}\|\nabla G\|_{L^{6}}^{1 / 2} d t \\
& \leq C(\bar{\rho}) \int_{0}^{\sigma(T)}\|\rho \dot{u}\|_{L^{2}}^{1 / 2}\|\nabla \dot{u}\|_{L^{2}}^{1 / 2} d t \\
& \leq C(\bar{\rho}) \int_{0}^{\sigma(T)}\left(\sigma\|\rho \dot{u}\|_{L^{2}}\right)^{1 / 4}\left(\sigma\|\rho \dot{u}\|_{L^{2}}^{2}\right)^{1 / 8}\left(\sigma\|\nabla \dot{u}\|_{L^{2}}^{2}\right)^{1 / 4} \sigma^{-5 / 8} d t \\
& \leq C(\bar{\rho}, M) C_{0}^{1 / 48} \int_{0}^{\sigma(T)}\left(\sigma\|\nabla \dot{u}\|_{L^{2}}^{2}\right)^{1 / 4} \sigma^{-5 / 8} d t \\
& \leq C(\bar{\rho}, M) C_{0}^{1 / 48}\left(\int_{0}^{\sigma(T)} \sigma\|\nabla \dot{u}\|_{L^{2}}^{2} d t\right)^{1 / 4}\left(\int_{0}^{\sigma(T)} \sigma^{-5 / 6} d t\right)^{3 / 4} \\
& \leq C(\bar{\rho}, M) C_{0}^{1 / 48},
\end{aligned}
$$

and

$$
\begin{aligned}
\int_{\sigma(T)}^{T}\|G\|_{L^{\infty}}^{2} d t & \leq C \int_{\sigma(T)}^{T}\|\nabla G\|_{L^{2}}\|\nabla G\|_{L^{6}} d t \\
& \leq C(\bar{\rho}) \int_{\sigma(T)}^{T}\|\rho \dot{u}\|_{L^{2}}\|\nabla \dot{u}\|_{L^{2}} d t \\
& \leq C(\bar{\rho}) \int_{\sigma(T)}^{T}\left(\left\|\rho^{1 / 2} \dot{u}\right\|_{L^{2}}^{2}+\|\nabla \dot{u}\|_{L^{2}}^{2}\right) d t \\
& \leq C(\bar{\rho}) C_{0}^{1 / 6} .
\end{aligned}
$$

Finally, denoting $D_{t} \rho=\rho_{t}+u \cdot \nabla \rho$ and expressing $(1.6)_{1}$ in terms of the Lagrangian coordinates, we obtain by (3.23) that

$$
\begin{aligned}
(2 \mu+\lambda) D_{t} \rho & =-R \rho(\rho-1)-R \rho^{2}(\theta-1)-\rho G \\
& \leq-R(\rho-1)+C(\bar{\rho})\|\theta-1\|_{L^{\infty}}+C(\bar{\rho})\|G\|_{L^{\infty}}
\end{aligned}
$$

which gives

$$
D_{t}(\rho-1)+\frac{R}{2 \mu+\lambda}(\rho-1) \leq C(\bar{\rho})\|\theta-1\|_{L^{\infty}}+C(\bar{\rho})\|G\|_{L^{\infty}} .
$$


Taking

$$
y=\rho-1, \quad \alpha=\frac{R}{2 \mu+\lambda}, \quad g(t)=C(\bar{\rho})\|\theta-1\|_{L^{\infty}}+C(\bar{\rho})\|G\|_{L^{\infty}}, \quad T_{1}=\sigma(T),
$$

in Lemma 2.5, we thus deduce from (3.96), (3.92)-(3.95), and (2.31) that

$$
\begin{aligned}
\rho & \leq \bar{\rho}+1+C\left(\|g\|_{L^{1}(0, \sigma(T))}+\|g\|_{L^{2}(\sigma(T), T)}\right) \\
& \leq \bar{\rho}+1+C(\bar{\rho}, M) C_{0}^{1 / 48} \leq \frac{3 \bar{\rho}}{2}
\end{aligned}
$$

provided

$$
C_{0} \leq \varepsilon_{4} \triangleq \min \left\{1,\left(\frac{\bar{\rho}-2}{2 C(\bar{\rho}, M)}\right)^{48}\right\} .
$$

We thus complete the proof of Lemma 3.7.

Next, the following Lemma 3.8 will give an estimate on $A_{4}(T)$, which together with Lemmas 3.2, 3.3, 3.6 and 3.7 finishes the proof of Proposition 3.1.

Lemma 3.8 Under the conditions of Proposition 3.1, there exists a positive constant $\varepsilon_{0}$ depending only on $\mu, \lambda, \kappa, R, \gamma, \bar{\rho}$, and $M$ such that if $(\rho, u, \theta)$ is a smooth solution of (1.6) (1.4) [1.7) on $\mathbb{R}^{3} \times(0, T]$ satisfying (3.6) with $K$ as in Lemma 3.2, the following estimate holds

$$
A_{4}(T) \leq C_{0}^{1 / 8}
$$

provided $C_{0} \leq \varepsilon_{0}$.

Proof. It follows from (3.41), (3.6), (3.73), and (3.82) that

$$
\begin{aligned}
& \int_{0}^{T} \sigma^{2}\left\|\nabla^{2} \theta\right\|_{L^{2}}^{2} d t \\
& \leq C(\bar{\rho}, M) \int_{0}^{T}\left(\sigma^{2}\|\rho \dot{\theta}\|_{L^{2}}^{2}+\sigma\|\rho \dot{u}\|_{L^{2}}^{2}+\|\nabla u\|_{L^{2}}^{2}+\|\nabla \theta\|_{L^{2}}^{2}+\sigma^{2}\|\rho-1\|_{L^{4}}^{4}\right) d t \\
& \leq C(\bar{\rho}, M) C_{0}^{1 / 6} .
\end{aligned}
$$

Applying the operator $\partial_{t}+\operatorname{div}(u \cdot)$ to $(1.6)_{3}$, we use $(1.6)_{1}$ to get

$$
\begin{aligned}
& \frac{R}{\gamma-1} \rho\left(\partial_{t} \dot{\theta}+u \cdot \nabla \dot{\theta}\right) \\
& =\kappa \Delta \dot{\theta}+\kappa\left(\operatorname{div} u \Delta \theta-\partial_{i}\left(\partial_{i} u \cdot \nabla \theta\right)-\partial_{i} u \cdot \nabla \partial_{i} \theta\right) \\
& \quad+\left(\lambda(\operatorname{div} u)^{2}+2 \mu|\mathfrak{D}(u)|^{2}\right) \operatorname{div} u+R \rho \theta \partial_{k} u^{l} \partial_{l} u^{k} \\
& \quad-R \rho \dot{\theta} \operatorname{div} u-R \rho \theta \operatorname{div} \dot{u}+2 \lambda\left(\operatorname{div} \dot{u}-\partial_{k} u^{l} \partial_{l} u^{k}\right) \operatorname{div} u \\
& \quad+\mu\left(\partial_{i} u^{j}+\partial_{j} u^{i}\right)\left(\partial_{i} \dot{u}^{j}+\partial_{j} \dot{u}^{i}-\partial_{i} u^{k} \partial_{k} u^{j}-\partial_{j} u^{k} \partial_{k} u^{i}\right) .
\end{aligned}
$$


Multiplying (3.99) by $\dot{\theta}$, we obtain after integration by parts that

$$
\begin{aligned}
& \frac{R}{2(\gamma-1)}\left(\int \rho|\dot{\theta}|^{2} d x\right)_{t}+\kappa\|\nabla \dot{\theta}\|_{L^{2}}^{2} \\
& \leq C \int|\nabla u|\left(\left|\nabla^{2} \theta\right||\dot{\theta}|+|\nabla \theta||\nabla \dot{\theta}|\right) d x+C(\bar{\rho}) \int|\nabla u|^{2}|\dot{\theta}|(|\nabla u|+|\theta-1|) d x \\
& \quad+C(\bar{\rho}) \int\left(|\nabla u|^{2}|\dot{\theta}|+\rho|\dot{\theta}|^{2}|\nabla u|\right) d x+C \int|\nabla \dot{u}| \rho|\dot{\theta}| d x \\
& \quad+C(\bar{\rho}) \int \rho|\theta-1\|\nabla \dot{u}\| \dot{\theta}| d x+C(\bar{\rho}) \int|\nabla u\|\nabla \dot{u}\| \dot{\theta}| d x \\
& \leq C\|\nabla u\|_{L^{2}}^{1 / 2}\|\nabla u\|_{L^{6}}^{1 / 2}\left\|\nabla^{2} \theta\right\|_{L^{2}}\|\nabla \dot{\theta}\|_{L^{2}} \\
& \quad+C(\bar{\rho})\|\nabla u\|_{L^{2}}\|\nabla u\|_{L^{6}}\left(\|\nabla u\|_{L^{6}}+\|\nabla \theta\|_{L^{2}}\right)\|\nabla \dot{\theta}\|_{L^{2}} \\
& \quad+C(\bar{\rho})\|\nabla u\|_{L^{6}}^{1 / 2}\|\nabla u\|_{L^{2}}^{1 / 2}\|\nabla \dot{\theta}\|_{L^{2}}\left(\|\nabla u\|_{L^{2}}+\|\rho \dot{\theta}\|_{L^{2}}\right) \\
& \quad+C(\bar{\rho})\|\nabla \dot{u}\|_{L^{2}}\|\rho \dot{\theta}\|_{L^{2}}+C(\bar{\rho})\left\|\rho^{1 / 2}(\theta-1)\right\|_{L^{2}}^{1 / 2}\|\nabla \theta\|_{L^{2}}^{1 / 2}\|\nabla \dot{u}\|_{L^{2}}\|\nabla \dot{\theta}\|_{L^{2}} \\
& \quad+C(\bar{\rho})\|\nabla u\|_{L^{2}}^{1 / 2}\|\nabla u\|_{L^{6}}^{1 / 2}\|\nabla \dot{u}\|_{L^{2}}\|\nabla \dot{\theta}\|_{L^{2}} .
\end{aligned}
$$

Multiplying (3.100) by $\sigma^{4}$ and integrating the resulting inequality over $(0, t)$, we obtain after integration by parts and using (3.43), (3.6), (3.24), and (3.98) that

$$
\begin{aligned}
& \frac{R}{2(\gamma-1)} \sigma^{4} \int \rho|\dot{\theta}|^{2} d x+\kappa \int_{0}^{t} \sigma^{4}\|\nabla \dot{\theta}\|_{L^{2}}^{2} d s \\
& \leq C \int_{0}^{\sigma(t)} \sigma^{2} \int \rho|\dot{\theta}|^{2} d x d s+C(\bar{\rho}) \int_{0}^{t} \sigma^{3}\left\|\nabla^{2} \theta\right\|_{L^{2}}\|\nabla \dot{\theta}\|_{L^{2}} d s \\
& \quad+C(\bar{\rho}) \int_{0}^{t} \sigma^{2}\|\nabla u\|_{L^{2}}\|\nabla \dot{\theta}\|_{L^{2}} d s+C(\bar{\rho}) \int_{0}^{t} \sigma^{3}\|\nabla \dot{\theta}\|_{L^{2}}\|\rho \dot{\theta}\|_{L^{2}} d s \\
& \quad+C(\bar{\rho}) \int_{0}^{t} \sigma^{3}\|\nabla \dot{u}\|_{L^{2}}\left\|\rho^{1 / 2} \dot{\theta}\right\|_{L^{2}} d s+C(\bar{\rho}) \int_{0}^{t} \sigma^{3}\|\nabla \dot{u}\|_{L^{2}}\|\nabla \dot{\theta}\|_{L^{2}} d s \\
& \leq C(\bar{\rho}) \int_{0}^{t}\left(\sigma^{2}\left\|\nabla^{2} \theta\right\|_{L^{2}}^{2}+\|\nabla u\|_{L^{2}}^{2}+\sigma^{2}\left\|\rho^{1 / 2} \dot{\theta}\right\|_{L^{2}}^{2}+\sigma^{2}\|\nabla \dot{u}\|_{L^{2}}^{2}\right) d s \\
& \quad+\frac{\kappa}{2} \int_{0}^{t} \sigma^{4}\|\nabla \dot{\theta}\|_{L^{2}}^{2} d s \\
& \leq C(\bar{\rho}, M) C_{0}^{1 / 6}+\frac{\kappa}{2} \int_{0}^{t} \sigma^{4}\|\nabla \dot{\theta}\|_{L^{2}}^{2} d s,
\end{aligned}
$$

which yields that

$$
\sup _{0 \leq t \leq T} \sigma^{4} \int \rho|\dot{\theta}|^{2} d x+\int_{0}^{T} \sigma^{4}\|\nabla \dot{\theta}\|_{L^{2}}^{2} d s \leq C(\bar{\rho}, M) C_{0}^{1 / 6} \leq C_{0}^{1 / 8}
$$

provided

$$
C_{0} \leq \varepsilon_{0} \triangleq \min _{1 \leq i \leq 5} \varepsilon_{i}, \quad \text { with } \quad \varepsilon_{5} \triangleq \min \left\{1,(C(\bar{\rho}, M))^{-24}\right\} .
$$

We thus finish the proof of Lemma 3.8 with $\varepsilon_{0}$ as in (3.101).

Finally, in the following Corollary [3.9, we summarize some estimates on $(\rho, u, \theta)$ which will be useful for higher order ones in the next section. 
Corollary 3.9 In addition to the conditions of Proposition 3.1, assume that $\left(\rho_{0}, u_{0}, \theta_{0}\right)$ satisfies (3.8) with $\varepsilon_{0}$ as in Proposition 3.1. Then there exists a positive constant $C$ depending only on $\mu, \lambda, \kappa, R, \gamma, \bar{\rho}$, and $M$ such that if $(\rho, u, \theta)$ is a smooth solution of (1.6) (1.4) (1.7) on $\mathbb{R}^{3} \times(0, T]$ satisfying (3.6) with $K$ as in Lemma 3.2, the following estimate holds

$$
\begin{aligned}
& \sup _{t \in(0, T]}\left(\sigma^{2}\|\nabla u\|_{L^{6}}^{2}+\sigma^{4}\|\theta-1\|_{H^{2}}^{2}\right) \\
& +\int_{0}^{T} \sigma^{2}\left(\|\rho-1\|_{L^{4}}^{4}+\|\nabla u\|_{L^{4}}^{4}+\|\nabla \theta\|_{H^{1}}^{2}+\left\|u_{t}\right\|_{L^{2}}^{2}+\sigma^{2}\left\|\theta_{t}\right\|_{H^{1}}^{2}\right) d t \leq C C_{0}^{1 / 8} .
\end{aligned}
$$

Proof. It follows from (3.6), (2.24), (3.10), (3.43), (3.44), (3.73), (3.82), and (3.98) that

$$
\begin{aligned}
& \sup _{t \in(0, T]}\left(\sigma^{2}\|\nabla u\|_{L^{6}}^{2}+\sigma^{4}\|\theta-1\|_{H^{2}}^{2}\right) \\
& +\int_{0}^{T} \sigma^{2}\left(\|\nabla u\|_{L^{4}}^{4}+\|\nabla \theta\|_{H^{1}}^{2}+\|\rho-1\|_{L^{4}}^{4}\right) d t \leq C C_{0}^{1 / 8},
\end{aligned}
$$

which together with (3.6) and (2.24) gives that

$$
\begin{aligned}
& \int_{0}^{T} \sigma^{2}\left\|u_{t}\right\|_{L^{2}}^{2} d t \\
& \leq C \int_{0}^{T} \sigma^{2}\|\dot{u}\|_{L^{2}}^{2} d t+C \int_{0}^{T} \sigma^{2}\|u \cdot \nabla u\|_{L^{2}}^{2} d t \\
& \leq C \int_{0}^{T} \sigma^{2} \int \rho|\dot{u}|^{2} d x d t+C \int_{0}^{T} \sigma^{2}\|\nabla \dot{u}\|_{L^{2}}^{2} d t \\
& \quad+C \int_{0}^{T} \sigma^{2}\|u\|_{L^{6}}^{2}\|\nabla u\|_{L^{3}}^{2} d t \\
& \leq C C_{0}^{1 / 6}, \\
& \int_{0}^{T} \sigma^{4}\left\|\theta_{t}\right\|_{L^{2}}^{2} d t \\
& \leq C \int_{0}^{T} \sigma^{4}\|\dot{\theta}\|_{L^{2}}^{2} d t+C \int_{0}^{T} \sigma^{4}\|u \cdot \nabla \theta\|_{L^{2}}^{2} d t \\
& \leq C \int_{0}^{T} \sigma^{4} \int \rho|\dot{\theta}|^{2} d x d t+C \int_{0}^{T} \sigma^{4}\|\nabla \dot{\theta}\|_{L^{2}}^{2} d t \\
& \quad+C \int_{0}^{T} \sigma^{4}\|u\|_{L^{6}}^{2}\|\nabla \theta\|_{L^{3}}^{2} d t \\
& \leq C C_{0}^{1 / 8}+C \int_{0}^{T}\|\nabla u\|_{L^{2}}^{2} d t \\
& \leq C C_{0}^{1 / 8},
\end{aligned}
$$

and

$$
\begin{aligned}
& \int_{0}^{T} \sigma^{4}\left\|\nabla \theta_{t}\right\|_{L^{2}}^{2} d t \\
& \leq C \int_{0}^{T} \sigma^{4}\|\nabla \dot{\theta}\|_{L^{2}}^{2} d t+C \int_{0}^{T} \sigma^{4}\|\nabla(u \cdot \nabla \theta)\|_{L^{2}}^{2} d t \\
& \leq C C_{0}^{1 / 8}+C \int_{0}^{T} \sigma^{4}\left(\|\nabla u\|_{L^{3}}^{2}+\|u\|_{L^{\infty}}^{2}\right)\left\|\nabla^{2} \theta\right\|_{L^{2}}^{2} d t \\
& \leq C C_{0}^{1 / 8} .
\end{aligned}
$$


We thus obtain (3.102) directly from (3.103) - (3.106) and finish the proof of Corollary 3.9 .

\section{A priori estimates (II): Higher-order estimates}

In this section, we will derive the higher order estimates of a smooth solution $(\rho, u, \theta)$ of (1.6) (1.4) (1.7) on $\mathbb{R}^{3} \times(0, T]$ with smooth $\left(\rho_{0}, u_{0}, \theta_{0}\right)$ satisfying (1.9) and (3.5). Moreover, we shall always assume that $(\rho, u, \theta)$ and $\left(\rho_{0}, u_{0}, \theta_{0}\right)$ satisfy respectively (3.6) and (3.8). To proceed, we define $\tilde{g_{1}}$ and $\tilde{g_{2}}$ as

$$
\tilde{g_{1}} \triangleq \rho_{0}^{-1 / 2}\left(-\mu \Delta u_{0}-(\mu+\lambda) \nabla \operatorname{div} u_{0}+R \nabla\left(\rho_{0} \theta_{0}\right)\right)
$$

and

$$
\tilde{g_{2}} \triangleq \rho_{0}^{-1 / 2}\left(\kappa \Delta \theta_{0}+\frac{\mu}{2}\left|\nabla u_{0}+\left(\nabla u_{0}\right)^{\operatorname{tr}}\right|^{2}+\lambda\left(\operatorname{div} u_{0}\right)^{2}\right),
$$

respectively. It thus follows from (1.9) and (3.5) that

$$
\tilde{g_{1}} \in L^{2}, \quad \tilde{g_{2}} \in L^{2} .
$$

From now on, the generic constant $C$ will depend only on

$$
T,\left\|\tilde{g_{1}}\right\|_{L^{2}},\left\|\tilde{g_{2}}\right\|_{L^{2}},\left\|u_{0}\right\|_{H^{2}},\left\|\rho_{0}-1\right\|_{H^{2} \cap W^{2, q}},\left\|\theta_{0}-1\right\|_{H^{2}},
$$

besides $\mu, \lambda, \kappa, R, \gamma, \bar{\rho}$, and $M$.

We begin with the important estimates on the spatial gradient of the smooth solution $(\rho, u, \theta)$.

Lemma 4.1 The following estimates hold

$$
\begin{aligned}
& \sup _{0 \leq t \leq T}\left(\left\|\rho^{1 / 2} \dot{u}\right\|_{L^{2}}+\|\theta-1\|_{H^{1}}\right)+\int_{0}^{T} \int \rho(\dot{\theta})^{2} d x d t \\
& \quad+\int_{0}^{T}\left(\|\nabla \dot{u}\|_{L^{2}}^{2}+\left\|\nabla^{2} \theta\right\|_{L^{2}}^{2}+\|\operatorname{div} u\|_{L^{\infty}}^{2}+\|\omega\|_{L^{\infty}}^{2}\right) d t \leq C, \\
& \sup _{0 \leq t \leq T}\left(\|\rho-1\|_{H^{1} \cap W^{1,6}}+\|u\|_{H^{2}}\right)+\int_{0}^{T}\|\nabla u\|_{L^{\infty}}^{3 / 2} d t \leq C .
\end{aligned}
$$

Proof. We first prove (4.4). Taking $m=0$ in (3.70) gives that, for $\varphi(t)$ as in (3.62),

$$
\begin{aligned}
& \varphi^{\prime}(t)+\int\left(\mu|\nabla \dot{u}|^{2}+\rho(\dot{\theta})^{2}\right) d x \\
& \leq C\left(\|\nabla u\|_{L^{2}}^{2}+\|\nabla \theta\|_{L^{2}}^{2}\right)\left(\left\|\rho^{1 / 2} \dot{u}\right\|_{L^{2}}^{2}+\|\nabla \theta\|_{L^{2}}^{2}+1\right)+C\|\nabla u\|_{L^{2}}\|\nabla u\|_{L^{6}}^{3} \\
& \leq C\left(\left\|\rho^{1 / 2} \dot{u}\right\|_{L^{2}}^{4}+\|\nabla \theta\|_{L^{2}}^{4}\right)+C \\
& \leq C\left(\left\|\rho^{1 / 2} \dot{u}\right\|_{L^{2}}^{2}+\|\nabla \theta\|_{L^{2}}^{2}\right) \varphi(t)+C\left(\left\|\rho^{1 / 2} \dot{u}\right\|_{L^{2}}^{2}+\|\nabla \theta\|_{L^{2}}^{2}\right)+C
\end{aligned}
$$

due to (3.6), (3.25), and (3.77). It follows from $(\underline{1.6})_{2},(\underline{3.5})$, and (4.1) that

$$
\lim _{t \rightarrow 0^{+}} \sqrt{\rho} \dot{u}(x, t)=\rho_{0}^{-1 / 2}\left(\mu \Delta u_{0}+(\mu+\lambda) \nabla \operatorname{div} u_{0}-R \nabla\left(\rho_{0} \theta_{0}\right)\right)=-\tilde{g_{1}},
$$


which together with (3.62), (3.63), (3.53), and (4.3) yields that

$$
\lim _{t \rightarrow 0^{+}}|\varphi(t)| \leq C\left\|\tilde{g}_{1}\right\|_{L^{2}}^{2}+C \leq C .
$$

Gronwall's inequality together with (4.6), (4.8), and (3.6) leads to

$$
\sup _{0 \leq t \leq T} \varphi(t)+\int_{0}^{T} \int\left(|\nabla \dot{u}|^{2}+\rho(\dot{\theta})^{2}\right) d x d t \leq C,
$$

which as well as (3.77) and (3.10) implies

$$
\sup _{0 \leq t \leq T}\left(\left\|\rho^{1 / 2} \dot{u}\right\|_{L^{2}}+\|\theta-1\|_{H^{1}}\right)+\int_{0}^{T} \int\left(|\nabla \dot{u}|^{2}+\rho(\dot{\theta})^{2}\right) d x d t \leq C .
$$

One thus deduces from (3.41), (4.9), (3.72), and (3.6) that

$$
\int_{0}^{T}\left\|\nabla^{2} \theta\right\|_{L^{2}}^{2} d t \leq C+C \int_{0}^{T}\left\|\rho^{1 / 2} \dot{\theta}\right\|_{L^{2}}^{2} d t \leq C,
$$

which together with (1.21), (2.27), (2.21), (4.9), and (3.6) gives

$$
\begin{aligned}
& \int_{0}^{T}\left(\|\operatorname{div} u\|_{L^{\infty}}^{2}+\|\omega\|_{L^{\infty}}^{2}\right) d t \\
& \leq C \int_{0}^{T}\left(\|G\|_{L^{\infty}}^{2}+\|\rho \theta-1\|_{L^{\infty}}^{2}+\|\omega\|_{L^{\infty}}^{2}\right) d t+C \\
& \leq C \int_{0}^{T}\left(\|G\|_{L^{6}}^{2}+\|\nabla G\|_{L^{6}}^{2}+\|\theta-1\|_{L^{\infty}}^{2}+\|\omega\|_{L^{6}}^{2}+\|\nabla \omega\|_{L^{6}}^{2}\right) d t+C \\
& \leq C \int_{0}^{T}\left(\|\nabla G\|_{L^{2}}^{2}+\|\rho \dot{u}\|_{L^{6}}^{2}+\left\|\nabla^{2} \theta\right\|_{L^{2}}^{2}+\|\nabla \omega\|_{L^{2}}^{2}\right) d t+C \\
& \leq C \int_{0}^{T}\left(\|\rho \dot{u}\|_{L^{2}}^{2}+\left\|\nabla^{2} \theta\right\|_{L^{2}}^{2}+\|\nabla \dot{u}\|_{L^{2}}^{2}\right) d t+C \\
& \leq C .
\end{aligned}
$$

This fact combining with (4.9) and (4.10) yields (4.4) directly.

Next, we will prove the key estimate (4.5). For $2 \leq p \leq 6,|\nabla \rho|^{p}$ satisfies

$$
\begin{aligned}
& \left(|\nabla \rho|^{p}\right)_{t}+\operatorname{div}\left(|\nabla \rho|^{p} u\right)+(p-1)|\nabla \rho|^{p} \operatorname{div} u \\
& +p|\nabla \rho|^{p-2}(\nabla \rho)^{t} \nabla u(\nabla \rho)+p \rho|\nabla \rho|^{p-2} \nabla \rho \cdot \nabla \operatorname{div} u=0 .
\end{aligned}
$$

Thus,

$$
\begin{aligned}
\partial_{t}\|\nabla \rho\|_{L^{p}} \leq & C\left(1+\|\nabla u\|_{L^{\infty}}\right)\|\nabla \rho\|_{L^{p}}+C\left\|\nabla^{2} u\right\|_{L^{p}} \\
\leq & C\left(1+\left\|\nabla^{2} \theta\right\|_{L^{2}}+\|\nabla u\|_{L^{\infty}}\right)\|\nabla \rho\|_{L^{p}} \\
& +C\left(1+\|\nabla \dot{u}\|_{L^{2}}+\left\|\nabla^{2} \theta\right\|_{L^{2}}\right)
\end{aligned}
$$

where we have used

$$
\begin{aligned}
\left\|\nabla^{2} u\right\|_{L^{p}} & \leq C\left(\|\rho \dot{u}\|_{L^{p}}+\|\nabla P\|_{L^{p}}\right) \\
& \leq C\left(\|\rho \dot{u}\|_{L^{2}}+\|\nabla \dot{u}\|_{L^{2}}+\|\nabla \theta\|_{L^{p}}+\|\theta\|_{L^{\infty}}\|\nabla \rho\|_{L^{p}}\right) \\
& \leq C\left(1+\|\nabla \dot{u}\|_{L^{2}}+\left\|\nabla^{2} \theta\right\|_{L^{2}}+\left(\left\|\nabla^{2} \theta\right\|_{L^{2}}+1\right)\|\nabla \rho\|_{L^{p}}\right),
\end{aligned}
$$


which comes from the standard $L^{p}$-estimate of the following elliptic system:

$$
\mu \Delta u+(\mu+\lambda) \nabla \operatorname{div} u=\rho \dot{u}+\nabla P, \quad u \rightarrow 0 \text { as }|x| \rightarrow \infty .
$$

It follows from Lemma 2.6 and (4.12) that

$$
\begin{aligned}
\|\nabla u\|_{L^{\infty}} \leq & C\left(\|\operatorname{div} u\|_{L^{\infty}}+\|\omega\|_{L^{\infty}}\right) \log \left(e+\left\|\nabla^{2} u\right\|_{L^{6}}\right)+C\|\nabla u\|_{L^{2}}+C \\
\leq & C\left(\|\operatorname{div} u\|_{L^{\infty}}+\|\omega\|_{L^{\infty}}\right) \log \left(e+\|\nabla \dot{u}\|_{L^{2}}+\left\|\nabla^{2} \theta\right\|_{L^{2}}\right) \\
& +C\left(\|\operatorname{div} u\|_{L^{\infty}}+\|\omega\|_{L^{\infty}}\right) \log \left(e+\left(e+\left\|\nabla^{2} \theta\right\|_{L^{2}}\right)\|\nabla \rho\|_{L^{6}}\right)+C \\
\leq & C\left(\|\operatorname{div} u\|_{L^{\infty}}+\|\omega\|_{L^{\infty}}\right) \log \left(e+\|\nabla \dot{u}\|_{L^{2}}+\left\|\nabla^{2} \theta\right\|_{L^{2}}\right) \\
& +C\left(\|\operatorname{div} u\|_{L^{\infty}}+\|\omega\|_{L^{\infty}}\right) \log \left(e+\|\nabla \rho\|_{L^{6}}\right)+C .
\end{aligned}
$$

Set

$$
\left\{\begin{aligned}
f(t) \triangleq & e+\|\nabla \rho\|_{L^{6}}, \\
g(t) \triangleq & 1+\left(\|\operatorname{div} u\|_{L^{\infty}}+\|\omega\|_{L^{\infty}}\right) \log \left(e+\|\nabla \dot{u}\|_{L^{2}}+\left\|\nabla^{2} \theta\right\|_{L^{2}}\right) \\
& +\|\nabla \dot{u}\|_{L^{2}}+\left\|\nabla^{2} \theta\right\|_{L^{2}} .
\end{aligned}\right.
$$

The combination of (4.14) with (4.11), where we set $p=6$, gives

$$
f^{\prime}(t) \leq C g(t) f(t)+C g(t) f(t) \ln f(t)+C g(t),
$$

which yields

$$
(\ln f(t))^{\prime} \leq C g(t)+C g(t) \ln f(t),
$$

due to $f(t)>1$. It thus follows from (4.15), (4.16), (4.4), and Gronwall's inequality that

$$
\sup _{0 \leq t \leq T} f(t) \leq C
$$

which shows

$$
\sup _{0 \leq t \leq T}\|\nabla \rho\|_{L^{6}} \leq C .
$$

Therefore, we deduce from (4.14), (4.17), and (4.4) that

$$
\int_{0}^{T}\|\nabla u\|_{L^{\infty}}^{3 / 2} d t \leq C
$$

Next, taking $p=2$ in (4.11), we get by using (4.18), (4.4) and Gronwall's inequality that

$$
\sup _{0 \leq t \leq T}\|\nabla \rho\|_{L^{2}} \leq C,
$$

which together with (4.4) and (4.17) gives

$$
\begin{aligned}
\sup _{0 \leq t \leq T}\|\nabla P\|_{L^{2}} & \leq C \sup _{0 \leq t \leq T}\left(\|\nabla \theta\|_{L^{2}}+\left(\|\nabla \rho\|_{L^{2}}+\|\theta-1\|_{L^{6}}\|\nabla \rho\|_{L^{3}}\right)\right) \\
& \leq C .
\end{aligned}
$$

This fact combining with (4.12) and (4.4) leads to

$$
\begin{aligned}
\sup _{0 \leq t \leq T}\left\|\nabla^{2} u\right\|_{L^{2}} & \leq C \sup _{0 \leq t \leq T}\left(\|\rho \dot{u}\|_{L^{2}}+\|\nabla P\|_{L^{2}}\right) \\
& \leq C .
\end{aligned}
$$

Hence, (4.5) follows directly from (3.9), (2.24), (4.21), and (4.18). The proof of Lemma 4.1 is completed. 
Lemma 4.2 The following estimates hold

$$
\begin{gathered}
\sup _{0 \leq t \leq T}\left(\left\|\rho_{t}\right\|_{H^{1}}+\|\theta-1\|_{H^{2}}+\|\rho-1\|_{H^{2}}+\|u\|_{H^{2}}\right) \\
+\int_{0}^{T}\left(\left\|u_{t}\right\|_{H^{1}}^{2}+\left\|\theta_{t}\right\|_{H^{1}}^{2}+\left\|\rho u_{t}\right\|_{H^{1}}^{2}+\left\|\rho \theta_{t}\right\|_{H^{1}}^{2}\right) d t \leq C, \\
\int_{0}^{T}\left(\left\|\left(\rho u_{t}\right)_{t}\right\|_{H^{-1}}^{2}+\left\|\left(\rho \theta_{t}\right)_{t}\right\|_{H^{-1}}^{2}\right) d t \leq C .
\end{gathered}
$$

Proof. First, it follows from (1.21), (4.4), (4.5), (2.25), and (2.23) that

$$
\begin{aligned}
\|\nabla u\|_{H^{2}} \leq & C\left(\|\operatorname{div} u\|_{H^{2}}+\|\omega\|_{H^{2}}\right) \\
\leq & C\left(\|G\|_{H^{2}}+\|\omega\|_{H^{2}}+\|\rho \theta-1\|_{H^{2}}\right) \\
\leq & C+C\|\nabla(\rho \dot{u})\|_{L^{2}}+C\|(\rho-1)(\theta-1)\|_{H^{2}} \\
& +C\|\rho-1\|_{H^{2}}+C\|\theta-1\|_{H^{2}} \\
\leq & C+C\left(\|\nabla \rho\|_{L^{3}}\|\dot{u}\|_{L^{6}}+\|\nabla \dot{u}\|_{L^{2}}\right)+C\|\rho-1\|_{H^{2}}\|\theta-1\|_{H^{2}} \\
& +C\left\|\nabla^{2} \rho\right\|_{L^{2}}+C\left\|\nabla^{2} \theta\right\|_{L^{2}} \\
\leq & C+C\left(1+\left\|\nabla^{2} \theta\right\|_{L^{2}}\right)\left\|\nabla^{2} \rho\right\|_{L^{2}}+C\|\nabla \dot{u}\|_{L^{2}}+C\left\|\nabla^{2} \theta\right\|_{L^{2}} .
\end{aligned}
$$

This fact combining with simple computations and (1.6) 1 gives

$$
\begin{aligned}
\frac{d}{d t}\left\|\nabla^{2} \rho\right\|_{L^{2}}^{2} \leq & C\left(1+\|\nabla u\|_{L^{\infty}}\right)\left\|\nabla^{2} \rho\right\|_{L^{2}}^{2}+C\|\nabla u\|_{H^{2}}^{2} \\
\leq & C\left(1+\|\nabla u\|_{L^{\infty}}+\left\|\nabla^{2} \theta\right\|_{L^{2}}\right)\left\|\nabla^{2} \rho\right\|_{L^{2}}^{2} \\
& +C\|\nabla \dot{u}\|_{L^{2}}^{2}+C\left\|\nabla^{2} \theta\right\|_{L^{2}}^{2}+C,
\end{aligned}
$$

which, together with (4.18), (4.4), and Gronwall's inequality, gives directly

$$
\sup _{0 \leq t \leq T}\left\|\nabla^{2} \rho\right\|_{L^{2}} \leq C
$$

Next, it follows from (1.6) $3,(3.5)$, and (4.2) that

$$
\begin{aligned}
& \frac{R}{\gamma-1} \lim _{t \rightarrow 0^{+}} \sqrt{\rho} \dot{\theta}(x, t) \\
& =-R \rho_{0}^{1 / 2} \theta_{0} \operatorname{div} u_{0}+\rho_{0}^{-1 / 2}\left(\kappa \Delta \theta_{0}+\frac{\mu}{2}\left|\nabla u_{0}+\left(\nabla u_{0}\right)^{\operatorname{tr}}\right|^{2}+\lambda\left(\operatorname{div} u_{0}\right)^{2}\right) \\
& =-R \rho_{0}^{1 / 2} \theta_{0} \operatorname{div} u_{0}+\tilde{g_{2}} .
\end{aligned}
$$

Integrating (3.100) over $(0, T)$ together with (4.5), (4.4), (3.25), and (4.27) leads to

$$
\begin{aligned}
& \sup _{0 \leq t \leq T} \int \rho(\dot{\theta})^{2} d x+\int_{0}^{T}\|\nabla \dot{\theta}\|_{L^{2}}^{2} d t \\
& \leq C \int_{0}^{T}\left(\left\|\nabla^{2} \theta\right\|_{L^{2}}^{2}+\|\nabla u\|_{L^{2}}^{2}+\left\|\rho^{1 / 2} \dot{\theta}\right\|_{L^{2}}^{2}+\|\nabla \dot{u}\|_{L^{2}}^{2}\right) d t \\
& \quad+\frac{1}{2} \int_{0}^{T}\|\nabla \dot{\theta}\|_{L^{2}}^{2} d t+C\left(\left\|\theta_{0}-1\right\|_{L^{6}}^{2}\left\|\nabla u_{0}\right\|_{L^{3}}^{2}+\left\|\nabla u_{0}\right\|_{L^{2}}^{2}\right)+C\left\|\tilde{g}_{2}\right\|_{L^{2}}^{2} \\
& \leq C+\frac{1}{2} \int_{0}^{T}\|\nabla \dot{\theta}\|_{L^{2}}^{2} d t
\end{aligned}
$$


which shows

$$
\sup _{0 \leq t \leq T} \int \rho(\dot{\theta})^{2} d x+\int_{0}^{T}\|\nabla \dot{\theta}\|_{L^{2}}^{2} d t \leq C .
$$

One thus deduces from (3.41), (4.28), (4.5), and (4.4) that

$$
\sup _{0 \leq t \leq T}\left\|\nabla^{2} \theta\right\|_{L^{2}} \leq C
$$

It follows from (4.4), (4.5), and (4.28) that

$$
\begin{aligned}
& \sup _{0 \leq t \leq T} \int \rho\left(\left|u_{t}\right|^{2}+\theta_{t}^{2}\right) d x+\int_{0}^{T}\left(\left\|\nabla u_{t}\right\|_{L^{2}}^{2}+\left\|\nabla \theta_{t}\right\|_{L^{2}}^{2}\right) d t \\
& \leq C \sup _{0 \leq t \leq T} \int \rho\left(|\dot{u}|^{2}+(\dot{\theta})^{2}\right) d x+C \sup _{0 \leq t \leq T} \int \rho\left(|u \cdot \nabla u|^{2}+|u \cdot \nabla \theta|^{2}\right) d x \\
& \quad+C \int_{0}^{T}\left(\|\nabla \dot{u}\|_{L^{2}}^{2}+\|\nabla \dot{\theta}\|_{L^{2}}^{2}+\left(\|\nabla u\|_{L^{3}}^{2}+\|u\|_{L^{\infty}}^{2}\right)\left(\left\|\nabla^{2} u\right\|_{L^{2}}^{2}+\left\|\nabla^{2} \theta\right\|_{L^{2}}^{2}\right)\right) d t \\
& \leq C,
\end{aligned}
$$

which together with (4.4) and (4.5) gives that

$$
\begin{aligned}
& \int_{0}^{T}\left(\left\|\nabla\left(\rho u_{t}\right)\right\|_{L^{2}}^{2}+\left\|\nabla\left(\rho \theta_{t}\right)\right\|_{L^{2}}^{2}\right) d t \\
& \leq C \int_{0}^{T}\left(\left\|\nabla u_{t}\right\|_{L^{2}}^{2}+\|\nabla \rho\|_{L^{3}}^{2}\left\|u_{t}\right\|_{L^{6}}^{2}+\left\|\nabla \theta_{t}\right\|_{L^{2}}^{2}+\|\nabla \rho\|_{L^{3}}^{2}\left\|\theta_{t}\right\|_{L^{6}}^{2}\right) d t \\
& \leq C .
\end{aligned}
$$

Next, one deduces from $(\underline{1.6})_{1}$ and (4.5) that

$$
\left\|\rho_{t}\right\|_{L^{2}} \leq C\|u\|_{L^{\infty}}\|\nabla \rho\|_{L^{2}}+C\|\nabla u\|_{L^{2}} \leq C .
$$

Applying $\nabla$ to $(1.6)_{1}$ yields that

$$
\nabla \rho_{t}+u^{i} \partial_{i} \nabla \rho+\nabla u^{i} \partial_{i} \rho+\nabla \rho \operatorname{div} u+\rho \nabla \operatorname{div} u=0
$$

which leads to

$$
\left\|\nabla \rho_{t}\right\|_{L^{2}} \leq C\|u\|_{L^{\infty}}\left\|\nabla^{2} \rho\right\|_{L^{2}}+C\|\nabla u\|_{L^{3}}\|\nabla \rho\|_{L^{6}}+C\left\|\nabla^{2} u\right\|_{L^{2}} \leq C,
$$

due to (4.5). The combination of (4.32) with (4.33) implies

$$
\sup _{0 \leq t \leq T}\left\|\rho_{t}\right\|_{H^{1}} \leq C,
$$

which together with (4.4), (4.26), (4.5), (4.30), (4.31), (4.29), and (2.24) gives (4.22).

Finally, differentiating $(1.6)_{3}$ with respect to $t$ yields that

$$
\begin{aligned}
\frac{R}{\gamma-1}\left(\rho \theta_{t}\right)_{t}= & -\frac{R}{\gamma-1}(\rho u \cdot \nabla \theta)_{t}-R(\rho \theta \operatorname{div} u)_{t}+\kappa \Delta \theta_{t} \\
& +\lambda\left((\operatorname{div} u)^{2}\right)_{t}+2 \mu\left(|\mathfrak{D}(u)|^{2}\right)_{t}
\end{aligned}
$$


It follows from (4.22) that

$$
\begin{aligned}
& \left\|(\rho u \cdot \nabla \theta)_{t}\right\|_{L^{2}} \\
& =\left\|\rho_{t} u \cdot \nabla \theta+\rho u_{t} \cdot \nabla \theta+\rho u \cdot \nabla \theta_{t}\right\|_{L^{2}} \\
& \leq C\left\|\rho_{t}\right\|_{L^{6}}\|\nabla \theta\|_{L^{3}}+C\left\|u_{t}\right\|_{L^{6}}\|\nabla \theta\|_{L^{3}}+C\|u\|_{L^{\infty}}\left\|\nabla \theta_{t}\right\|_{L^{2}} \\
& \leq C+C\left\|u_{t}\right\|_{H^{1}}+C\left\|\theta_{t}\right\|_{H^{1}}, \\
& \quad\left\|(\rho \theta \operatorname{div} u)_{t}\right\|_{L^{2}} \leq C+C\left\|u_{t}\right\|_{H^{1}}+C\left\|\theta_{t}\right\|_{H^{1}},
\end{aligned}
$$

and

$$
\begin{aligned}
& \left\|\left((\operatorname{div} u)^{2}\right)_{t}\right\|_{L^{6 / 5}}+\left\|\left(|\mathfrak{D}(u)|^{2}\right)_{t}\right\|_{L^{6 / 5}} \\
& \leq C\|\nabla u\|_{L^{3}}\left\|\nabla u_{t}\right\|_{L^{2}} \\
& \leq C+C\left\|u_{t}\right\|_{H^{1}} .
\end{aligned}
$$

Thus, it follows directly from (4.34)-(4.37) and (4.22) that

$$
\int_{0}^{T}\left\|\left(\rho \theta_{t}\right)\right\|_{H^{-1}}^{2} d t \leq C
$$

Similarly, we have

$$
\int_{0}^{T}\left\|\left(\rho u_{t}\right)_{t}\right\|_{H^{-1}}^{2} d t \leq C,
$$

which combining with (4.38) implies (4.23). The proof of Lemma 4.2 is completed.

Lemma 4.3 The following estimate holds:

$$
\sup _{0 \leq t \leq T} \sigma\left(\left\|\nabla u_{t}\right\|_{L^{2}}^{2}+\left\|\rho_{t t}\right\|_{L^{2}}^{2}\right)+\int_{0}^{T} \sigma \int \rho\left|u_{t t}\right|^{2} d x d t \leq C .
$$

Proof. Multiplying (2.6) by $u_{t t}$, one gets after integrating the resulting equality by parts that

$$
\begin{aligned}
& \frac{1}{2} \frac{d}{d t} \int\left(\mu\left|\nabla u_{t}\right|^{2}+(\mu+\lambda)\left(\operatorname{div} u_{t}\right)^{2}\right) d x+\int \rho\left|u_{t t}\right|^{2} d x \\
& =\frac{d}{d t}\left(-\frac{1}{2} \int \rho_{t}\left|u_{t}\right|^{2} d x-\int \rho_{t} u \cdot \nabla u \cdot u_{t} d x+\int P_{t} \operatorname{div} u_{t} d x\right) \\
& \quad+\frac{1}{2} \int \rho_{t t}\left|u_{t}\right|^{2} d x+\int\left(\rho_{t} u \cdot \nabla u\right)_{t} \cdot u_{t} d x-\int \rho u_{t} \cdot \nabla u \cdot u_{t t} d x \\
& \quad-\int \rho u \cdot \nabla u_{t} \cdot u_{t t} d x-\int\left(P_{t t}-\kappa(\gamma-1) \Delta \theta_{t}\right) \operatorname{div} u_{t} d x \\
& \quad+\kappa(\gamma-1) \int \nabla \theta_{t} \cdot \nabla \operatorname{div} u_{t} d x \\
& \triangleq
\end{aligned}
$$

We estimate each term on the righthand side of (4.40) as follows: 
First, it follows from (1.6) 1 , (4.22), (4.30), and (2.24) that

$$
\begin{aligned}
&\left|I_{0}\right|=\left.\left|-\frac{1}{2} \int \rho_{t}\right| u_{t}\right|^{2} d x-\int \rho_{t} u \cdot \nabla u \cdot u_{t} d x+\int P_{t} \operatorname{div} u_{t} d x \mid \\
& \leq\left.\left|\int \operatorname{div}(\rho u)\right| u_{t}\right|^{2} d x \mid+C\left\|\rho_{t}\right\|_{L^{3}}\|u \cdot \nabla u\|_{L^{2}}\left\|u_{t}\right\|_{L^{6}}+C\left\|(\rho \theta)_{t}\right\|_{L^{2}}\left\|\nabla u_{t}\right\|_{L^{2}} \\
& \leq C \int \rho\left|u\left\|u_{t}|| \nabla u_{t} \mid d x+C\left(1+\left\|\rho^{1 / 2} \theta_{t}\right\|_{L^{2}}+\left\|\rho_{t}\right\|_{L^{2}}\|\theta\|_{L^{\infty}}\right)\right\| \nabla u_{t} \|_{L^{2}}\right. \\
& \leq C\|u\|_{L^{6}}\left\|\rho^{1 / 2} u_{t}\right\|_{L^{2}}^{1 / 2}\left\|u_{t}\right\|_{L^{6}}^{1 / 2}\left\|\nabla u_{t}\right\|_{L^{2}}+C\left\|\nabla u_{t}\right\|_{L^{2}} \\
& \leq \frac{\mu}{4}\left\|\nabla u_{t}\right\|_{L^{2}}^{2}+C, \\
& 2\left|I_{1}\right|=\left.\left|\int \rho_{t t}\right| u_{t}\right|^{2} d x \mid \\
& \leq C\left\|\rho_{t t}\right\|_{L^{2}}\left\|u_{t}\right\|_{L^{2}}^{1 / 2}\left\|u_{t}\right\|_{L^{6}}^{3 / 2} \\
& \leq C\left\|\rho_{t t}\right\|_{L^{2}}\left(1+\left\|\nabla u_{t}\right\|_{L^{2}}\right)^{1 / 2}\left\|\nabla u_{t}\right\|_{L^{2}}^{3 / 2} \\
& \leq C\left\|\rho_{t t}\right\|_{L^{2}}^{2}+C\left\|\nabla u_{t}\right\|_{L^{2}}^{4}+C,
\end{aligned}
$$

and

$$
\begin{aligned}
\left|I_{2}\right|= & \left|\int\left(\rho_{t} u \cdot \nabla u\right)_{t} \cdot u_{t} d x\right| \\
= & \left|\int\left(\rho_{t t} u \cdot \nabla u \cdot u_{t}+\rho_{t} u_{t} \cdot \nabla u \cdot u_{t}+\rho_{t} u \cdot \nabla u_{t} \cdot u_{t}\right) d x\right| \\
\leq & C\left\|\rho_{t t}\right\|_{L^{2}}\|u \cdot \nabla u\|_{L^{3}}\left\|u_{t}\right\|_{L^{6}}+C\left\|\rho_{t}\right\|_{L^{2}}\left\|\left.u_{t}\right|^{2}\right\|_{L^{3}}\|\nabla u\|_{L^{6}} \\
& +C\left\|\rho_{t}\right\|_{L^{3}}\|u\|_{L^{\infty}}\left\|\nabla u_{t}\right\|_{L^{2}}\left\|u_{t}\right\|_{L^{6}} \\
\leq & C\left\|\rho_{t t}\right\|_{L^{2}}^{2}+C\left\|\nabla u_{t}\right\|_{L^{2}}^{2} .
\end{aligned}
$$

Next, Cauchy's inequality gives

$$
\begin{aligned}
\left|I_{3}\right|+\left|I_{4}\right| & =\left|\int \rho u_{t} \cdot \nabla u \cdot u_{t t} d x\right|+\left|\int \rho u \cdot \nabla u_{t} \cdot u_{t t} d x\right| \\
& \leq C\left\|\rho^{1 / 2} u_{t t}\right\|_{L^{2}}\left(\left\|u_{t}\right\|_{L^{6}}\|\nabla u\|_{L^{3}}+\|u\|_{L^{\infty}}\left\|\nabla u_{t}\right\|_{L^{2}}\right) \\
& \leq \frac{1}{4}\left\|\rho^{1 / 2} u_{t t}\right\|_{L^{2}}^{2}+C\left\|\nabla u_{t}\right\|_{L^{2}}^{2} .
\end{aligned}
$$

Next, it follows from (4.22) that

$$
\begin{aligned}
\left\|\nabla P_{t}\right\|_{L^{2}} \leq & C\left\|\nabla\left(\rho \theta_{t}+\theta \rho_{t}\right)\right\|_{L^{2}} \\
\leq & C\|\nabla \rho\|_{L^{3}}\left\|\theta_{t}\right\|_{L^{6}}+C\left\|\nabla \theta_{t}\right\|_{L^{2}}+C\|\nabla \theta\|_{L^{6}}\left\|\rho_{t}\right\|_{L^{3}} \\
& +C\|\theta\|_{L^{\infty}}\left\|\nabla \rho_{t}\right\|_{L^{2}} \\
\leq & +C\left\|\nabla \theta_{t}\right\|_{L^{2}}
\end{aligned}
$$

This fact together with (3.26) and (4.22) gives

$$
\begin{aligned}
& \left\|P_{t t}-\kappa(\gamma-1) \Delta \theta_{t}\right\|_{L^{2}} \\
& \leq C\left\|(u \cdot \nabla P)_{t}\right\|_{L^{2}}+C\left\|(P \operatorname{div} u)_{t}\right\|_{L^{2}}+C\left\|\left|\nabla u\left\|\nabla u_{t} \mid\right\|_{L^{2}}\right.\right. \\
& \leq C\left\|u_{t}\right\|_{L^{6}}\|\nabla P\|_{L^{3}}+C\|u\|_{L^{\infty}}\left\|\nabla P_{t}\right\|_{L^{2}}+C\left\|P_{t}\right\|_{L^{6}}\|\nabla u\|_{L^{3}} \\
& +C\|P\|_{L^{\infty}}\left\|\nabla u_{t}\right\|_{L^{2}}+C\|\nabla u\|_{L^{\infty}}\left\|\nabla u_{t}\right\|_{L^{2}} \\
& \leq C+C\left(1+\|\nabla u\|_{L^{\infty}}\right)\left\|\nabla u_{t}\right\|_{L^{2}}+C\left\|\nabla \theta_{t}\right\|_{L^{2}}
\end{aligned}
$$


which directly yields

$$
\begin{aligned}
\left|I_{5}\right| & =\left|\int\left(P_{t t}-\kappa(\gamma-1) \Delta \theta_{t}\right) \operatorname{div} u_{t} d x\right| \\
& \leq\left\|P_{t t}-\kappa(\gamma-1) \Delta \theta_{t}\right\|_{L^{2}}\left\|\operatorname{div} u_{t}\right\|_{L^{2}} \\
& \leq C+C\left(1+\|\nabla u\|_{L^{\infty}}\right)\left\|\nabla u_{t}\right\|_{L^{2}}^{2}+C\left\|\nabla \theta_{t}\right\|_{L^{2}}^{2} .
\end{aligned}
$$

Finally, it follows from (4.45), (4.22), and the standard $L^{2}$-estimate for elliptic system (2.6) that

$$
\begin{aligned}
\left\|\nabla^{2} u_{t}\right\|_{L^{2}} \leq & C\left\|\rho u_{t t}+\rho_{t} u_{t}+\rho_{t} u \cdot \nabla u+\rho u_{t} \cdot \nabla u+\rho u \cdot \nabla u_{t}+\nabla P_{t}\right\|_{L^{2}} \\
\leq & C\left\|\rho^{1 / 2} u_{t t}\right\|_{L^{2}}+C\left\|\rho_{t}\right\|_{L^{3}}\left\|u_{t}\right\|_{L^{6}}+C\left\|\rho_{t}\right\|_{L^{3}}\|u\|_{L^{\infty}}\|\nabla u\|_{L^{6}} \\
& +C\left\|u_{t}\right\|_{L^{6}}\|\nabla u\|_{L^{3}}+C\|u\|_{L^{\infty}}\left\|\nabla u_{t}\right\|_{L^{2}}+C\left\|\nabla P_{t}\right\|_{L^{2}} \\
\leq & C+C\left\|\rho^{1 / 2} u_{t t}\right\|_{L^{2}}+C\left\|\nabla \theta_{t}\right\|_{L^{2}}+C\left\|\nabla u_{t}\right\|_{L^{2}} .
\end{aligned}
$$

The combination of this fact with Cauchy inequality thus leads to

$$
\begin{aligned}
\left|I_{6}\right| & =\left|\kappa(\gamma-1) \int \nabla \theta_{t} \cdot \nabla \operatorname{div} u_{t} d x\right| \\
& \leq C\left\|\nabla^{2} u_{t}\right\|_{L^{2}}\left\|\nabla \theta_{t}\right\|_{L^{2}} \\
& \leq C\left(1+\left\|\rho^{1 / 2} u_{t t}\right\|_{L^{2}}+\left\|\nabla \theta_{t}\right\|_{L^{2}}+\left\|\nabla u_{t}\right\|_{L^{2}}\right)\left\|\nabla \theta_{t}\right\|_{L^{2}} \\
& \leq C+\frac{1}{4}\left\|\rho^{1 / 2} u_{t t}\right\|_{L^{2}}^{2}+C\left\|\nabla \theta_{t}\right\|_{L^{2}}^{2}+C\left\|\nabla u_{t}\right\|_{L^{2}}^{2} .
\end{aligned}
$$

Substituting all the estimates (4.42)-(4.48) into (4.40) gives

$$
\begin{aligned}
& \frac{d}{d t} \int\left(\mu\left|\nabla u_{t}\right|^{2}+(\mu+\lambda)\left(\operatorname{div} u_{t}\right)^{2}-2 I_{0}\right) d x+\int \rho\left|u_{t t}\right|^{2} d x \\
& \leq C\left\|\rho_{t t}\right\|_{L^{2}}^{2}+C\left(1+\|\nabla u\|_{L^{\infty}}+\left\|\nabla u_{t}\right\|_{L^{2}}^{2}\right)\left\|\nabla u_{t}\right\|_{L^{2}}^{2}+C\left\|\nabla \theta_{t}\right\|_{L^{2}}^{2}+C .
\end{aligned}
$$

Then, differentiating $(1.6)_{1}$ with respect to $t$ shows

$$
\rho_{t t}+\rho_{t} \operatorname{div} u+\rho \operatorname{div} u_{t}+u_{t} \cdot \nabla \rho+u \cdot \nabla \rho_{t}=0
$$

which combining with (4.22) implies

$$
\begin{aligned}
\left\|\rho_{t t}\right\|_{L^{2}} & \leq C\left(\left\|\rho_{t}\right\|_{L^{6}}\|\nabla u\|_{L^{3}}+\left\|\nabla u_{t}\right\|_{L^{2}}+\left\|u_{t}\right\|_{L^{6}}\|\nabla \rho\|_{L^{3}}+\left\|\nabla \rho_{t}\right\|_{L^{2}}\right) \\
& \leq C+C\left\|\nabla u_{t}\right\|_{L^{2}} .
\end{aligned}
$$

This fact together with (4.30) yields

$$
\int_{0}^{T}\left\|\rho_{t t}\right\|_{L^{2}}^{2} d t \leq C .
$$

One thus deduces from (4.49), (4.41), (4.22), (4.5), (4.51), and Gronwall's inequality that

$$
\sup _{0 \leq t \leq T} \sigma\left\|\nabla u_{t}\right\|_{L^{2}}^{2}+\int_{0}^{T} \sigma \int \rho\left|u_{t t}\right|^{2} d x d t \leq C,
$$

which together with (4.50) gives (4.39). We complete the proof of Lemma 4.3, 
Lemma 4.4 For $q \in(3,6)$ as in Theorem 1.1, it holds that

$$
\begin{aligned}
& \sup _{0 \leq t \leq T}\left(\|\rho-1\|_{W^{2, q}}+\sigma\|u\|_{H^{3}}^{2}\right) \\
& +\int_{0}^{T}\left(\|u\|_{H^{3}}^{2}+\left\|\nabla^{2} u\right\|_{W^{1, q}}^{p_{0}}+\sigma\left\|\nabla u_{t}\right\|_{H^{1}}^{2}\right) d t \leq C,
\end{aligned}
$$

where

$$
p_{0} \triangleq \frac{1}{2} \min \left\{\frac{5 q-6}{3(q-2)}, \frac{9 q-6}{5 q-6}\right\} \in(1,7 / 6)
$$

Proof. First, it follows from (4.24), (4.22), and (4.39) that

$$
\sup _{0 \leq t \leq T} \sigma\|u\|_{H^{3}}^{2}+\int_{0}^{T}\|u\|_{H^{3}}^{2} d t \leq C .
$$

The standard $H^{1}$-estimate for elliptic problem (3.40) together with (4.22) leads to

$$
\begin{aligned}
\left\|\nabla^{2} \theta\right\|_{H^{1}} \leq & C\left\|\nabla\left(\rho \theta_{t}\right)\right\|_{L^{2}}+C\left(\|\nabla(\rho u \cdot \nabla \theta)\|_{L^{2}}+\|\nabla(\rho \theta \operatorname{div} u)\|_{L^{2}}\right) \\
& +C\|\mid \nabla u\| \nabla^{2} u\|\|_{L^{2}}+C \\
\leq & C\left(\|\nabla \rho\|_{L^{3}}+1\right)\left\|\nabla \theta_{t}\right\|_{L^{2}}+C\left(1+\|\rho-1\|_{H^{2}}\right)\left(1+\|\theta-1\|_{H^{2}}\right)\|u\|_{H^{2}} \\
& +C\|\nabla u\|_{L^{6}}\left\|\nabla^{2} u\right\|_{L^{2}}^{1 / 2}\left\|\nabla^{2} u\right\|_{L^{6}}^{1 / 2}+C \\
\leq & C+C\left\|\nabla \theta_{t}\right\|_{L^{2}}+C\left\|\nabla^{2} u\right\|_{L^{6}}^{1 / 2},
\end{aligned}
$$

which combining with (4.22), (4.47), (4.39), and (4.54) yields that

$$
\int_{0}^{T}\left(\|\theta-1\|_{H^{3}}^{2}+\|u\|_{H^{3}}^{2}+\sigma\left\|\nabla u_{t}\right\|_{H^{1}}^{2}\right) d t \leq C .
$$

Next, it follows from standard $W^{1, p}$-estimate for elliptic systems (2.25) that

$$
\begin{aligned}
\left\|\nabla^{2} u\right\|_{W^{1, q}} \leq & C\|u\|_{H^{3}}+C\left\|\nabla^{2} \operatorname{div} u\right\|_{L^{q}}+C\left\|\nabla^{2} \omega\right\|_{L^{q}}+C \\
\leq & C\|u\|_{H^{3}}+C\|\nabla(\rho \dot{u})\|_{L^{q}}+C\left\|\nabla^{2}(\rho \theta)\right\|_{L^{q}}+C \\
\leq & C\|u\|_{H^{3}}+C\|\nabla(\rho \dot{u})\|_{L^{q}}+C\left\|\theta \nabla^{2} \rho\right\|_{L^{q}}+C\|\nabla \rho \nabla \theta\|_{L^{q}} \\
& +C\left\|\rho \nabla^{2} \theta\right\|_{L^{q}}+C \\
\leq & C\|u\|_{H^{3}}+C\|\nabla(\rho \dot{u})\|_{L^{q}}+C\left\|\nabla^{2} \rho\right\|_{L^{q}}+C\left\|\nabla^{2} \theta\right\|_{H^{1}}+C .
\end{aligned}
$$

Applying operator $\Delta$ to $(1.6)_{1}$ gives

$$
(\Delta \rho)_{t}+\operatorname{div}(u \Delta \rho)+\operatorname{div}(\rho \Delta u)+2 \operatorname{div}\left(\partial_{i} \rho \cdot \partial_{i} u\right)=0 .
$$

Multiplying (4.58) by $q|\Delta \rho|^{q-2} \Delta \rho$ and integrating the resulting equality over $\mathbb{R}^{3}$, we obtain after using (4.22) and (4.57) that

$$
\begin{aligned}
\left(\|\Delta \rho\|_{L^{q}}^{q}\right)_{t} & \leq C\left(1+\|\nabla u\|_{L^{\infty}}\right)\|\Delta \rho\|_{L^{q}}^{q}+C\left(\|\nabla \rho\|_{L^{q}}+1\right)\left\|\nabla^{2} u\right\|_{W^{1, q}}\|\Delta \rho\|_{L^{q}}^{q-1} \\
& \leq C\left(1+\|u\|_{H^{3}}+\|\nabla(\rho \dot{u})\|_{L^{q}}+\left\|\nabla^{2} \theta\right\|_{H^{1}}\right)\left(\|\Delta \rho\|_{L^{q}}^{q}+1\right) .
\end{aligned}
$$


Note that (4.22) and (4.39) give

$$
\begin{aligned}
\|\nabla(\rho \dot{u})\|_{L^{q}} \leq & C\|\nabla \rho\|_{L^{6}}\|\nabla \dot{u}\|_{L^{2}}^{q /(3(q-2))}\|\nabla \dot{u}\|_{L^{q}}^{2(q-3) /(3(q-2))}+C\|\nabla \dot{u}\|_{L^{q}} \\
\leq & C\|\nabla \dot{u}\|_{L^{2}}+C\left\|\nabla u_{t}\right\|_{L^{q}}+C\|\nabla(u \cdot \nabla u)\|_{L^{q}} \\
\leq & C\left\|\nabla u_{t}\right\|_{L^{2}}+C+C\left\|\nabla u_{t}\right\|_{L^{2}}^{(6-q) /(2 q)}\left\|\nabla u_{t}\right\|_{L^{6}}^{3(q-2) /(2 q)} \\
& +C\|\nabla u\|_{L^{6}}^{(6-q) / q}\|u\|_{H^{3}}^{3(q-2) / q}+C\|u\|_{L^{\infty}}\left\|\nabla^{2} u\right\|_{L^{q}} \\
\leq & C \sigma^{-1 / 2}+C\|u\|_{H^{3}}^{3(q-2) / q}+C \sigma^{-1 / 2}\left(\sigma\left\|\nabla u_{t}\right\|_{H^{1}}^{2}\right)^{3(q-2) /(4 q)}
\end{aligned}
$$

which combining with (4.56) shows that, for $p_{0}$ as in (4.53),

$$
\int_{0}^{T}\|\nabla(\rho \dot{u})\|_{L^{q}}^{p_{0}} d t \leq C
$$

Applying Gronwall's inequality to (4.59), we obtain after using (4.56) and (4.61) that

$$
\sup _{0 \leq t \leq T}\|\Delta \rho\|_{L^{q}} \leq C
$$

which combining with (4.22), (4.54), (4.57), (4.61), and (4.56) gives (4.52). We finish the proof of Lemma 4.4 .

Lemma 4.5 For $q \in(3,6)$ as in Theorem 1.1, the following estimate holds

$$
\sup _{0 \leq t \leq T} \sigma\left(\left\|\theta_{t}\right\|_{H^{1}}+\left\|\nabla^{3} \theta\right\|_{L^{2}}+\left\|u_{t}\right\|_{H^{2}}+\|u\|_{W^{3, q}}\right)+\int_{0}^{T} \sigma^{2}\left\|\nabla u_{t t}\right\|_{L^{2}}^{2} d t \leq C .
$$

Proof. First, multiplying (2.12) by $u_{t t}$ and integrating the resulting equality over $\mathbb{R}^{3}$, one gets after integration by parts that

$$
\begin{aligned}
& \frac{1}{2} \frac{d}{d t} \int \rho\left|u_{t t}\right|^{2} d x+\int\left(\mu\left|\nabla u_{t t}\right|^{2}+(\mu+\lambda)\left(\operatorname{div} u_{t t}\right)^{2}\right) d x \\
& =-4 \int u_{t t}^{i} \rho u \cdot \nabla u_{t t}^{i} d x-\int(\rho u)_{t} \cdot\left[\nabla\left(u_{t} \cdot u_{t t}\right)+2 \nabla u_{t} \cdot u_{t t}\right] d x \\
& \quad-\int\left(\rho_{t t} u+2 \rho_{t} u_{t}\right) \cdot \nabla u \cdot u_{t t} d x-\int \rho u_{t t} \cdot \nabla u \cdot u_{t t} d x+\int P_{t t} \operatorname{div} u_{t t} d x \\
& \triangleq \sum_{i=1}^{5} J_{i} .
\end{aligned}
$$

Hölder's inequality and (4.22) give

$$
\begin{aligned}
\left|J_{1}\right| & \leq C\left\|\rho^{1 / 2} u_{t t}\right\|_{L^{2}}\left\|\nabla u_{t t}\right\|_{L^{2}}\|u\|_{L^{\infty}} \\
& \leq \frac{\mu}{8}\left\|\nabla u_{t t}\right\|_{L^{2}}^{2}+C\left\|\rho^{1 / 2} u_{t t}\right\|_{L^{2}}^{2} .
\end{aligned}
$$

It follows from (4.30), (4.22), (4.39), and (4.52) that

$$
\begin{aligned}
\left|J_{2}\right| & \leq C\left(\left\|\rho u_{t}\right\|_{L^{3}}+\left\|\rho_{t} u\right\|_{L^{3}}\right)\left(\left\|\nabla u_{t t}\right\|_{L^{2}}\left\|u_{t}\right\|_{L^{6}}+\left\|u_{t t}\right\|_{L^{6}}\left\|\nabla u_{t}\right\|_{L^{2}}\right) \\
& \leq C\left(\left\|\rho^{1 / 2} u_{t}\right\|_{L^{2}}^{1 / 2}\left\|u_{t}\right\|_{L^{6}}^{1 / 2}+\left\|\rho_{t}\right\|_{L^{6}}\|u\|_{L^{6}}\right)\left\|\nabla u_{t t}\right\|_{L^{2}}\left\|\nabla u_{t}\right\|_{L^{2}} \\
& \leq \frac{\mu}{8}\left\|\nabla u_{t t}\right\|_{L^{2}}^{2}+C\left\|\nabla u_{t}\right\|_{L^{2}}^{3}+C \\
& \leq \frac{\mu}{8}\left\|\nabla u_{t t}\right\|_{L^{2}}^{2}+C \sigma^{-3 / 2},
\end{aligned}
$$




$$
\begin{aligned}
\left|J_{3}\right| & \leq C\left(\left\|\rho_{t t}\right\|_{L^{2}}\|u\|_{L^{6}}+\left\|\rho_{t}\right\|_{L^{2}}\left\|u_{t}\right\|_{L^{6}}\right)\|\nabla u\|_{L^{6}}\left\|u_{t t}\right\|_{L^{6}} \\
& \leq \frac{\mu}{8}\left\|\nabla u_{t t}\right\|_{L^{2}}^{2}+C\left\|\rho_{t t}\right\|_{L^{2}}^{2}+C\left\|\nabla u_{t}\right\|_{L^{2}}^{2}
\end{aligned}
$$

and

$$
\begin{aligned}
\left|J_{4}\right|+\left|J_{5}\right| \leq & C\left\|\rho u_{t t}\right\|_{L^{2}}\|\nabla u\|_{L^{3}}\left\|u_{t t}\right\|_{L^{6}}+C\left\|\left(\rho_{t} \theta+\rho \theta_{t}\right)_{t}\right\|_{L^{2}}\left\|\nabla u_{t t}\right\|_{L^{2}} \\
\leq & \frac{\mu}{8}\left\|\nabla u_{t t}\right\|_{L^{2}}^{2}+C\left\|\rho^{1 / 2} u_{t t}\right\|_{L^{2}}^{2}+C\left\|\rho_{t t} \theta\right\|_{L^{2}}^{2}+C\left\|\rho_{t} \theta_{t}\right\|_{L^{2}}^{2} \\
& +C\left\|\rho^{1 / 2} \theta_{t t}\right\|_{L^{2}}^{2} \\
\leq & \frac{\mu}{8}\left\|\nabla u_{t t}\right\|_{L^{2}}^{2}+C\left\|\rho^{1 / 2} u_{t t}\right\|_{L^{2}}^{2}+C\left\|\rho_{t t}\right\|_{L^{2}}^{2}+C\left\|\nabla \theta_{t}\right\|_{L^{2}}^{2} \\
& +C\left\|\rho^{1 / 2} \theta_{t t}\right\|_{L^{2}}^{2} .
\end{aligned}
$$

Substituting (4.64)-(4.67) into (4.63) yields

$$
\begin{aligned}
& \frac{d}{d t} \int \rho\left|u_{t t}\right|^{2} d x+\mu \int\left|\nabla u_{t t}\right|^{2} d x \\
& \leq C \sigma^{-3 / 2}+C\left\|\rho^{1 / 2} u_{t t}\right\|_{L^{2}}^{2}+C\left\|\rho_{t t}\right\|_{L^{2}}^{2}+C\left\|\nabla \theta_{t}\right\|_{L^{2}}^{2}+C{ }_{3}\left\|\rho^{1 / 2} \theta_{t t}\right\|_{L^{2}}^{2} .
\end{aligned}
$$

Then, to estimate the last term on the righthand side of (4.68), we multiply (2.9) by $\theta_{t t}$ and integrate the resulting equality over $\mathbb{R}^{3}$ to get

$$
\begin{aligned}
& \left(\frac{\kappa(\gamma-1)}{2 R}\left\|\nabla \theta_{t}\right\|_{L^{2}}^{2}+H_{0}\right)_{t}+\int \rho \theta_{t t}^{2} d x \\
& =\frac{1}{2} \int \rho_{t t}\left(\theta_{t}^{2}+2(u \cdot \nabla \theta+(\gamma-1) \theta \operatorname{div} u) \theta_{t}\right) d x \\
& \quad+\int \rho_{t}(u \cdot \nabla \theta+(\gamma-1) \theta \operatorname{div} u)_{t} \theta_{t} d x \\
& \quad-\int \rho(u \cdot \nabla \theta+(\gamma-1) \theta \operatorname{div} u)_{t} \theta_{t t} d x \\
& \quad-\frac{\gamma-1}{R} \int\left(\lambda(\operatorname{div} u)^{2}+2 \mu|\mathfrak{D}(u)|^{2}\right)_{t t} \theta_{t} d x \\
& \triangleq \sum_{i=1}^{4} H_{i},
\end{aligned}
$$

where

$$
\begin{aligned}
H_{0} \triangleq & \frac{1}{2} \int \rho_{t} \theta_{t}^{2} d x+\int \rho_{t}(u \cdot \nabla \theta+(\gamma-1) \theta \operatorname{div} u) \theta_{t} d x \\
& -\frac{\gamma-1}{R} \int\left(\lambda(\operatorname{div} u)^{2}+2 \mu|\mathfrak{D}(u)|^{2}\right)_{t} \theta_{t} d x
\end{aligned}
$$

satisfies

$$
\begin{aligned}
\left|H_{0}\right| \leq & C \int \rho\left|u\left\|\theta_{t}\right\| \nabla \theta_{t}\right| d x+C\left\|\rho_{t}\right\|_{L^{3}}\left\|\theta_{t}\right\|_{L^{6}}\left(\|\nabla \theta\|_{L^{2}}+\|\nabla u\|_{L^{2}}\right) \\
& +C\|\nabla u\|_{L^{3}}\left\|\nabla u_{t}\right\|_{L^{2}}\left\|\theta_{t}\right\|_{L^{6}} \\
\leq & C\left\|\rho \theta_{t}\right\|_{L^{2}}\|u\|_{L^{\infty}}\left\|\nabla \theta_{t}\right\|_{L^{2}}+C\left\|\nabla \theta_{t}\right\|_{L^{2}}+C\left\|\nabla \theta_{t}\right\|_{L^{2}}\left\|\nabla u_{t}\right\|_{L^{2}} \\
\leq & \frac{\kappa(\gamma-1)}{4 R}\left\|\nabla \theta_{t}\right\|_{L^{2}}^{2}+C \sigma^{-1},
\end{aligned}
$$


due to (1.6) 1 , (4.22), (4.30), and (4.39). Note that (4.30) and (2.24) yield

$$
\left\|\theta_{t}\right\|_{L^{2}} \leq C+C\left\|\nabla \theta_{t}\right\|_{L^{2}}
$$

which as well as (4.22) gives

$$
\begin{aligned}
\left|H_{1}\right| & \leq C\left\|\rho_{t t}\right\|_{L^{2}}\left(\left\|\theta_{t}\right\|_{L^{2}}^{1 / 2}\left\|\theta_{t}\right\|_{L^{6}}^{3 / 2}+\left\|\theta_{t}\right\|_{L^{6}}\left(\|u \cdot \nabla \theta\|_{L^{3}}+\|\theta \operatorname{div} u\|_{L^{3}}\right)\right) \\
& \leq C\left\|\nabla \theta_{t}\right\|_{L^{2}}^{4}+C\left\|\rho_{t t}\right\|_{L^{2}}^{2}+C .
\end{aligned}
$$

It follows from (4.22) that

$$
\begin{aligned}
& \left\|(u \cdot \nabla \theta+(\gamma-1) \theta \operatorname{div} u)_{t}\right\|_{L^{2}} \\
& \leq C\left(\left\|u_{t}\right\|_{L^{6}}\|\nabla \theta\|_{L^{3}}+\|u\|_{L^{\infty}}\left\|\nabla \theta_{t}\right\|_{L^{2}}+\left\|\theta_{t}\right\|_{L^{6}}\|\nabla u\|_{L^{3}}+\|\theta\|_{L^{\infty}}\left\|\nabla u_{t}\right\|_{L^{2}}\right) \\
& \leq C\left\|\nabla \theta_{t}\right\|_{L^{2}}+C\left\|\nabla u_{t}\right\|_{L^{2}}
\end{aligned}
$$

which together with (4.22) shows

$$
\begin{aligned}
\left|H_{2}\right|+\left|H_{3}\right| & \leq C\left(\left\|\nabla \theta_{t}\right\|_{L^{2}}+\left\|\nabla u_{t}\right\|_{L^{2}}\right)\left(\left\|\rho_{t}\right\|_{L^{3}}\left\|\theta_{t}\right\|_{L^{6}}+\left\|\rho \theta_{t t}\right\|_{L^{2}}\right) \\
& \leq \frac{1}{2} \int \rho \theta_{t t}^{2} d x+C\left\|\nabla \theta_{t}\right\|_{L^{2}}^{2}+C\left\|\nabla u_{t}\right\|_{L^{2}}^{2} .
\end{aligned}
$$

One deduces from (4.22) and (4.39) that

$$
\begin{aligned}
\left|H_{4}\right| & \leq C \int\left(\left|\nabla u_{t}\right|^{2}+\left|\nabla u \| \nabla u_{t t}\right|\right)\left|\theta_{t}\right| d x \\
& \leq C\left(\left\|\nabla u_{t}\right\|_{L^{2}}^{3 / 2}\left\|\nabla u_{t}\right\|_{L^{6}}^{1 / 2}+\|\nabla u\|_{L^{3}}\left\|\nabla u_{t t}\right\|_{L^{2}}\right)\left\|\theta_{t}\right\|_{L^{6}} \\
& \leq \delta\left\|\nabla u_{t t}\right\|_{L^{2}}^{2}+C\left\|\nabla^{2} u_{t}\right\|_{L^{2}}^{2}+C(\delta)\left\|\nabla \theta_{t}\right\|_{L^{2}}^{2}+C \sigma^{-2}\left\|\nabla u_{t}\right\|_{L^{2}}^{2} .
\end{aligned}
$$

Substituting (4.72), (4.74), and (4.75) into (4.69) gives

$$
\begin{aligned}
& \left(\frac{\kappa(\gamma-1)}{2 R}\left\|\nabla \theta_{t}\right\|_{L^{2}}^{2}+H_{0}\right)_{t}+\frac{1}{2} \int \rho \theta_{t t}^{2} d x \\
& \leq \delta\left\|\nabla u_{t t}\right\|_{L^{2}}^{2}+C(\delta)\left\|\nabla \theta_{t}\right\|_{L^{2}}^{4}+C\left\|\nabla^{2} u_{t}\right\|_{L^{2}}^{2}+C \sigma^{-2}\left\|\nabla u_{t}\right\|_{L^{2}}^{2} \\
& \quad+C\left\|\rho_{t t}\right\|_{L^{2}}^{2}+C .
\end{aligned}
$$

Finally, for $C_{3}$ as in (4.68), adding (4.76) multiplied by $2\left(C_{3}+1\right)$ to (4.68), we obtain after choosing $\delta$ suitably small that

$$
\begin{aligned}
& \left(2\left(C_{3}+1\right)\left(\frac{\kappa(\gamma-1)}{2 R}\left\|\nabla \theta_{t}\right\|_{L^{2}}^{2}+H_{0}\right)+\int \rho\left|u_{t t}\right|^{2} d x\right)_{t} \\
& \quad+\int \rho \theta_{t t}^{2} d x+\frac{\mu}{2} \int\left|\nabla u_{t t}\right|^{2} d x \\
& \leq C \sigma^{-3 / 2}+C\left\|\nabla \theta_{t}\right\|_{L^{2}}^{4}+C\left\|\nabla^{2} u_{t}\right\|_{L^{2}}^{2}+C \sigma^{-2}\left\|\nabla u_{t}\right\|_{L^{2}}^{2}+C\left\|\rho_{t t}\right\|_{L^{2}}^{2} \\
& \quad+C\left\|\rho^{1 / 2} u_{t t}\right\|_{L^{2}}^{2} .
\end{aligned}
$$

Multiplying (4.77) by $\sigma^{2}$ and integrating the resulting inequality over $(0, T)$, we obtain by using (4.70), (4.52), (4.39), (4.30), and Gronwall's inequality that

$$
\sup _{0 \leq t \leq T} \sigma^{2} \int\left(\left|\nabla \theta_{t}\right|^{2}+\rho\left|u_{t t}\right|^{2}\right) d x+\int_{0}^{T} \sigma^{2} \int\left(\rho \theta_{t t}^{2}+\left|\nabla u_{t t}\right|^{2}\right) d x d t \leq C
$$


which together with (4.47), (4.39), (4.55), (4.54), (4.57), and (4.60) gives

$$
\sup _{0 \leq t \leq T} \sigma\left(\left\|\nabla u_{t}\right\|_{H^{1}}+\left\|\nabla^{3} \theta\right\|_{L^{2}}+\left\|\nabla^{2} u\right\|_{W^{1, q}}\right) \leq C .
$$

We thus derive (4.62) from (4.78), (4.71), (4.79), and (4.52). The proof of Lemma 4.5 is completed.

Lemma 4.6 The following estimate holds

$$
\sup _{0 \leq t \leq T} \sigma^{2}\left(\left\|\nabla^{2} \theta\right\|_{H^{2}}+\left\|\theta_{t}\right\|_{H^{2}}\right)+\int_{0}^{T} \sigma^{4}\left\|\nabla \theta_{t t}\right\|_{L^{2}}^{2} d t \leq C .
$$

Proof. Multiplying (2.16) by $\theta_{t t}$ and integrating the resulting equality over $\mathbb{R}^{3}$ yield that

$$
\begin{aligned}
\frac{1}{2} & \frac{d}{d t} \int \rho\left(\theta_{t t}\right)^{2} d x+\frac{\kappa(\gamma-1)}{R} \int\left|\nabla \theta_{t t}\right|^{2} d x \\
= & -4 \int \theta_{t t} \rho u \cdot \nabla \theta_{t t} d x-\int \rho_{t t}\left(\theta_{t}+u \cdot \nabla \theta+(\gamma-1) \theta \operatorname{div} u\right) \theta_{t t} d x \\
& -2 \int \rho_{t}(u \cdot \nabla \theta+(\gamma-1) \theta \operatorname{div} u)_{t} \theta_{t t} d x \\
& -\int \rho\left(u_{t t} \cdot \nabla \theta+2 u_{t} \cdot \nabla \theta_{t}+(\gamma-1)(\theta \operatorname{div} u)_{t t}\right) \theta_{t t} d x \\
& +\frac{\gamma-1}{R} \int\left(\lambda(\operatorname{div} u)^{2}+2 \mu|\mathfrak{D}(u)|^{2}\right)_{t t} \theta_{t t} d x \\
\triangleq & \sum_{i=1}^{5} K_{i} .
\end{aligned}
$$

Hölder's inequality and (4.22) give

$$
\begin{aligned}
\sigma^{4}\left|K_{1}\right| & \leq C \sigma^{4}\left\|\rho^{1 / 2} \theta_{t t}\right\|_{L^{2}}\left\|\nabla \theta_{t t}\right\|_{L^{2}}\|u\|_{L^{\infty}} \\
& \leq \delta \sigma^{4}\left\|\nabla \theta_{t t}\right\|_{L^{2}}^{2}+C(\delta) \sigma^{4}\left\|\rho^{1 / 2} \theta_{t t}\right\|_{L^{2}}^{2} .
\end{aligned}
$$

It follows from (4.39), (4.62), and (4.22) that

$$
\begin{aligned}
\sigma^{4}\left|K_{2}\right| \leq & C \sigma^{4}\left\|\rho_{t t}\right\|_{L^{2}}\left\|\theta_{t t}\right\|_{L^{6}}\left(\left\|\theta_{t}\right\|_{H^{1}}+\|\nabla \theta\|_{L^{6}}\|u\|_{L^{6}}+\|\nabla u\|_{L^{3}}\|\theta\|_{L^{\infty}}\right) \\
\leq & C \sigma^{2}\left\|\nabla \theta_{t t}\right\|_{L^{2}} \\
\leq & C \delta \sigma^{4}\left\|\nabla \theta_{t t}\right\|_{L^{2}}^{2}+C(\delta), \\
\sigma^{4}\left|K_{4}\right| \leq & C \sigma^{4}\left\|\theta_{t t}\right\|_{L^{6}}\left(\|\nabla \theta\|_{L^{3}}\left\|\rho u_{t t}\right\|_{L^{2}}+\left\|\nabla \theta_{t}\right\|_{L^{2}}\left\|\rho u_{t}\right\|_{L^{2}}^{1 / 2}\left\|u_{t}\right\|_{L^{6}}^{1 / 2}\right) \\
& +C \sigma^{4}\left\|\theta_{t t}\right\|_{L^{6}}\left(\|\nabla u\|_{L^{3}}\left\|\rho \theta_{t t}\right\|_{L^{2}}+\left\|\nabla u_{t}\right\|_{L^{2}}\left\|\rho \theta_{t}\right\|_{L^{2}}^{1 / 2}\left\|\theta_{t}\right\|_{L^{6}}^{1 / 2}\right) \\
& +C \sigma^{4}\|\theta\|_{L^{\infty}}\left\|\rho \theta_{t t}\right\|_{L^{2}}\left\|\nabla u_{t t}\right\|_{L^{2}} \\
\leq & \delta \sigma^{4}\left\|\nabla \theta_{t t}\right\|_{L^{2}}^{2}+C(\delta) \sigma^{4}\left(\left\|\rho \theta_{t t}\right\|_{L^{2}}^{2}+\left\|\nabla u_{t t}\right\|_{L^{2}}^{2}\right)+C(\delta),
\end{aligned}
$$

and

$$
\begin{aligned}
\sigma^{4}\left|K_{5}\right| & \leq C \sigma^{4}\left\|\theta_{t t}\right\|_{L^{6}}\left(\left\|\nabla u_{t}\right\|_{L^{2}}^{3 / 2}\left\|\nabla u_{t}\right\|_{L^{6}}^{1 / 2}+\|\nabla u\|_{L^{3}}\left\|\nabla u_{t t}\right\|_{L^{2}}\right) \\
& \leq \delta \sigma^{4}\left\|\nabla \theta_{t t}\right\|_{L^{2}}^{2}+C(\delta) \sigma^{4}\left\|\nabla u_{t t}\right\|_{L^{2}}^{2}+C(\delta) .
\end{aligned}
$$


For $K_{3}$, one deduces from (4.73), (4.62), and (4.22) that

$$
\begin{aligned}
\sigma^{4}\left|K_{3}\right| & \leq C \sigma^{4}\left\|\rho_{t}\right\|_{L^{3}}\left\|\theta_{t t}\right\|_{L^{6}}\left(\left\|\nabla u_{t}\right\|_{L^{2}}+\left\|\nabla \theta_{t}\right\|_{L^{2}}\right) \\
& \leq C \delta \sigma^{4}\left\|\nabla \theta_{t t}\right\|_{L^{2}}^{2}+C(\delta) .
\end{aligned}
$$

Multiplying (4.81) by $\sigma^{4}$, substituting (4.82)-(4.86) into the resulting equality and choosing $\delta$ suitably small lead to

$$
\begin{aligned}
& \frac{d}{d t} \int \sigma^{4} \rho\left(\theta_{t t}\right)^{2} d x+\frac{\kappa(\gamma-1)}{R} \sigma^{4} \int\left|\nabla \theta_{t t}\right|^{2} d x \\
& \leq C \sigma^{2}\left(\left\|\rho^{1 / 2} \theta_{t t}\right\|_{L^{2}}^{2}+\left\|\nabla u_{t t}\right\|_{L^{2}}^{2}\right)+C
\end{aligned}
$$

which together with (4.78) gives

$$
\sup _{0 \leq t \leq T} \sigma^{4} \int \rho\left|\theta_{t t}\right|^{2} d x+\int_{0}^{T} \sigma^{4} \int\left|\nabla \theta_{t t}\right|^{2} d x d t \leq C .
$$

Applying the standard $L^{2}$-estimate to (2.9), by (4.73), (4.22), (4.87), and (4.62), we get

$$
\begin{aligned}
& \sup _{0 \leq t \leq T} \sigma^{2}\left\|\nabla^{2} \theta_{t}\right\|_{L^{2}} \\
& \leq C \sup _{0 \leq t \leq T} \sigma^{2}\left(\left\|\rho \theta_{t t}\right\|_{L^{2}}+\left\|\rho_{t}\right\|_{L^{3}}\left\|\theta_{t}\right\|_{L^{6}}+\left\|\rho_{t}\right\|_{L^{6}}\left(\|\nabla \theta\|_{L^{3}}+\|\nabla u\|_{L^{3}}\right)\right) \\
& \quad+C \sup _{0 \leq t \leq T} \sigma^{2}\left(\left\|\nabla \theta_{t}\right\|_{L^{2}}+\left\|\nabla u_{t}\right\|_{L^{2}}+\|\nabla u\|_{L^{3}}\left\|\nabla u_{t}\right\|_{L^{6}}\right)+C \\
& \leq C .
\end{aligned}
$$

It follows from the standard $H^{2}$-estimate of (3.40) that

$$
\begin{aligned}
\left\|\nabla^{2} \theta\right\|_{H^{2}} \leq & C\left(\left\|\rho \theta_{t}\right\|_{H^{2}}+\|\rho u \cdot \nabla \theta\|_{H^{2}}+\|\rho \theta \operatorname{div} u\|_{H^{2}}+\left\|\left.\nabla u\right|^{2}\right\|_{H^{2}}\right) \\
\leq & C\left(\left(\|\rho-1\|_{H^{2}}+1\right)\left\|\theta_{t}\right\|_{H^{2}}+\left(\|\rho-1\|_{H^{2}}+1\right)\|u\|_{H^{2}}\|\nabla \theta\|_{H^{2}}\right) \\
& +C\left(\|\rho \theta-1\|_{H^{2}}+1\right)\|\operatorname{div} u\|_{H^{2}}+C\|\nabla u\|_{H^{2}}^{2} \\
\leq & C+C\left\|\nabla^{3} u\right\|_{L^{2}}+C\left\|\nabla^{3} \theta\right\|_{L^{2}}+C\left\|\theta_{t}\right\|_{H^{2}}
\end{aligned}
$$

due to (2.23) and (4.22). This fact as well as (4.52), (4.62), (4.88), and (4.87) implies (4.80). The proof of Lemma 4.6 is completed.

\section{Proofs of Theorems 1.1 and 1.2}

With all the a priori estimates in Sections 3 and 4 at hand, we are ready to prove the main results of this paper in this section.

Proposition 5.1 For given numbers $M>0$ (not necessarily small), $\bar{\rho}>2$, assume that $\left(\rho_{0}, u_{0}, \theta_{0}\right)$ satisfies (2.1), (3.5), and (3.8). Then there exists a unique classical solution $(\rho, u, \theta)$ of (1.6) (1.4) (1.7) in $\mathbb{R}^{3} \times(0, \infty)$ satisfying (2.3)-(2.5) with $T_{0}$ replaced by any $T \in(0, \infty)$. Moreover, (3.9), (3.6), and (3.102) hold for any $T \in(0, \infty)$. 
Proof. First, standard local existence result, Lemma 2.1, applies to show that the Cauchy problem (1.6) (1.4) (1.7) with initial data $\left(\rho_{0}, u_{0}, \theta_{0}\right)$ has a unique local solution $(\rho, u, \theta)$, defined up to a positive $T_{0}$ which may depend on $\inf _{x \in \mathbb{R}^{3}} \rho_{0}(x)$, and satisfying (2.3) -(2.5), and $\inf _{x \in \mathbb{R}^{3}} \rho_{0}(x) / 4 \leq \rho \leq 2 \bar{\rho}$. One deduces from (3.1)-(3.5) that

$$
A_{1}(0) \leq M, \quad A_{2}(0) \leq C_{0} \leq C_{0}^{1 / 4}, \quad A_{3}(0)=A_{4}(0)=0, \quad \rho_{0}<\bar{\rho} .
$$

Then there exists a $T_{1} \in\left(0, T_{0}\right]$ such that (3.6) holds for $T=T_{1}$. We set

$$
T^{*}=\sup \left\{T \mid \sup _{t \in[0, T]}\|(\rho-1, u, \theta-1)\|_{H^{3}}<\infty\right\},
$$

and

$$
T_{*}=\sup \left\{T \leq T^{*} \mid(\underline{3.6}) \text { holds }\right\} .
$$

Then $T^{*} \geq T_{*} \geq T_{1}>0$. We claim that

$$
T_{*}=\infty
$$

Otherwise, $T_{*}<\infty$. Proposition 3.1 implies that (3.7) holds for all $0<T<T_{*}$, which together with (3.8) yields Lemmas 4.14.6) still hold for all $0<T<T_{*}$. Note here that all constants $C$ in Lemmas 4.14 4.6 depend on $T_{*}$ and $\inf _{x \in \mathbb{R}^{3}} \rho_{0}(x)$, and are in fact independent of $T$.

Next, we claim that there exists a positive constant $\tilde{C}$ which may depend on $T_{*}$ and $\inf _{x \in \mathbb{R}^{3}} \rho_{0}(x)$ such that, for all $0<T<T_{*}$,

$$
\sup _{0 \leq t \leq T}\|\rho-1\|_{H^{3}} \leq \tilde{C},
$$

which together with Lemmas 4.44 .6 and (3.5) gives

$$
\left\|\left(\rho\left(x, T_{*}\right)-1, u\left(x, T_{*}\right), \theta\left(x, T_{*}\right)-1\right)\right\|_{H^{3}} \leq \tilde{C}, \quad \inf _{x \in \mathbb{R}^{3}} \rho\left(x, T_{*}\right)>0 .
$$

This fact as well as Lemma 2.1 implies that there exists some $T^{* *}>T_{*}$, such that (3.6) holds for $T=T^{* *}$, which contradicts (5.2). Hence, we obtain (5.3) which together with Lemma 2.1 finishes the proof of Proposition 5.1 .

Finally, it remains to prove (5.4). It follows from (3.5), (1.6) 2 , and (2.2) that we can define

$$
u_{t}(\cdot, 0) \triangleq-u_{0} \cdot \nabla u_{0}+\rho_{0}^{-1}\left(\mu \Delta u_{0}+(\mu+\lambda) \nabla \operatorname{div} u_{0}-R \nabla\left(\rho_{0} \theta_{0}\right)\right),
$$

which together with (2.1) gives

$$
\left\|\nabla u_{t}(\cdot, 0)\right\|_{L^{2}} \leq \tilde{C}
$$

It thus follows from (4.49), (5.5), (4.41), (4.51), (4.22), (4.5), and Gronwall's inequality that

$$
\sup _{0 \leq t \leq T}\left\|\nabla u_{t}\right\|_{L^{2}}+\int_{0}^{T} \int \rho\left|u_{t t}\right|^{2} d x d t \leq \tilde{C},
$$


which as well as (4.24) and (4.22) yields

$$
\sup _{0 \leq t \leq T}\|u\|_{H^{3}} \leq \tilde{C}
$$

This combining with (4.55), (4.47), (5.6), and (4.22) gives

$$
\int_{0}^{T}\left(\left\|\nabla^{3} \theta\right\|_{L^{2}}^{2}+\left\|\nabla u_{t}\right\|_{H^{1}}^{2}\right) d t \leq \tilde{C} .
$$

Applying the $H^{2}$-estimate to elliptic systems (2.25) leads to

$$
\begin{aligned}
\left\|\nabla^{2} u\right\|_{H^{2}} & \leq \tilde{C}\|\nabla \operatorname{div} u\|_{H^{2}}+\tilde{C}\|\nabla \omega\|_{H^{2}} \\
& \leq \tilde{C}\|\rho \dot{u}\|_{H^{2}}+\tilde{C}\|\nabla P\|_{H^{2}} \\
& \leq \tilde{C}+\tilde{C}\left\|\nabla^{2} u_{t}\right\|_{L^{2}}+\tilde{C}\left\|\nabla^{3} \rho\right\|_{L^{2}}+\tilde{C}\left\|\nabla^{3} \theta\right\|_{L^{2}},
\end{aligned}
$$

where one has used (4.22) and the following simple facts:

$$
\begin{aligned}
\left\|\rho u_{t}\right\|_{H^{2}} & \leq \tilde{C}\left\|(\rho-1) u_{t}\right\|_{H^{2}}+\tilde{C}\left\|u_{t}\right\|_{H^{2}} \\
& \leq \tilde{C}\|\rho-1\|_{H^{2}}\left\|u_{t}\right\|_{H^{2}}+\tilde{C}\left\|\nabla^{2} u_{t}\right\|_{L^{2}}+\tilde{C} \\
& \leq \tilde{C}+\tilde{C}\left\|\nabla^{2} u_{t}\right\|_{L^{2}}, \\
\|\rho u \cdot \nabla u\|_{H^{2}} & \leq \tilde{C}\left(\|(\rho-1) u\|_{H^{2}}+\|u\|_{H^{2}}\right)\|\nabla u\|_{H^{2}} \\
& \leq \tilde{C}\|\rho-1\|_{H^{2}}\|u\|_{H^{2}}+\tilde{C} \\
& \leq \tilde{C}
\end{aligned}
$$

and

$$
\begin{aligned}
\left\|\nabla^{3}(\rho \theta)\right\|_{L^{2}} \leq \tilde{C}\left\|\nabla^{3} \rho\right\|_{L^{2}}\|\theta\|_{L^{\infty}}+\tilde{C}\left\|\nabla^{2} \rho\right\|_{L^{6}}\|\nabla \theta\|_{L^{3}} \\
\\
+\tilde{C}\|\nabla \rho\|_{L^{3}}\left\|\nabla^{2} \theta\right\|_{L^{6}}+\tilde{C}\left\|\nabla^{3} \theta\right\|_{L^{2}} \\
\leq \tilde{C}\left\|\nabla^{3} \rho\right\|_{L^{2}}+\tilde{C}\left\|\nabla^{3} \theta\right\|_{L^{2}}
\end{aligned}
$$

due to (2.23), (4.22), (5.6), (4.39), and (5.7). Standard calculations lead to

$$
\begin{aligned}
\left(\left\|\nabla^{3} \rho\right\|_{L^{2}}^{2}\right)_{t} \\
\leq \tilde{C}\left(\left\|\left|\nabla^{3} u\left\|\nabla \rho\left|\left\|_{L^{2}}+\right\|\right| \nabla^{2} u\right\| \nabla^{2} \rho\|\|_{L^{2}}+\left\|\left|\nabla u\left\|\nabla^{3} \rho \mid\right\|_{L^{2}}\right)\right\| \nabla^{3} \rho \|_{L^{2}}\right.\right.\right. \\
\quad+\tilde{C}\left\|\nabla^{4} u\right\|_{L^{2}}\left\|\nabla^{3} \rho\right\|_{L^{2}} \\
\leq \tilde{C}\left(\left\|\nabla^{3} u\right\|_{L^{2}}\|\nabla \rho\|_{H^{2}}+\left\|\nabla^{2} u\right\|_{L^{3}}\left\|\nabla^{2} \rho\right\|_{L^{6}}+\|\nabla u\|_{L^{\infty}}\left\|\nabla^{3} \rho\right\|_{L^{2}}\right)\left\|\nabla^{3} \rho\right\|_{L^{2}} \\
\quad+\tilde{C}\left(1+\left\|\nabla^{2} u_{t}\right\|_{L^{2}}+\left\|\nabla^{3} \rho\right\|_{L^{2}}+\left\|\nabla^{3} \theta\right\|_{L^{2}}\right)\left\|\nabla^{3} \rho\right\|_{L^{2}} \\
\leq \tilde{C}+\tilde{C}\left\|\nabla^{2} u_{t}\right\|_{L^{2}}^{2}+\tilde{C}\left\|\nabla^{3} \rho\right\|_{L^{2}}^{2}+\tilde{C}\left\|\nabla^{3} \theta\right\|_{L^{2}}^{2},
\end{aligned}
$$

due to (4.22), (5.7), and (5.9). It thus follows from (5.10), (5.8), and Gronwall's inequality that

$$
\sup _{0 \leq t \leq T}\left\|\nabla^{3} \rho\right\|_{L^{2}} \leq \tilde{C}
$$

which together with (4.22) gives (5.4). The proof of Proposition 5.1 is completed.

With Proposition 5.1 at hand, we are now in a position to prove our main results, Theorems 1.1 and 1.2 . 
Proof of Theorem 1.1. Let $\left(\rho_{0}, u_{0}, \theta_{0}\right)$ satisfying (1.9)-(1.12) be initial data as described in Theorem 1.1. Assume that $C_{0}$ satisfies (1.13), where

$$
\varepsilon \triangleq \varepsilon_{0} / 2
$$

with $\varepsilon_{0}$ as in Proposition 3.1. For constants

$$
\delta, \eta \in\left(0, \min \left\{1, \bar{\rho}-\sup _{x \in \mathbb{R}^{3}} \rho_{0}(x)\right\}\right),
$$

we define

$$
\rho_{0}^{\delta, \eta} \triangleq \frac{j_{\delta} * \rho_{0}+\eta}{1+\eta}, \quad u_{0}^{\delta, \eta} \triangleq j_{\delta} * u_{0}, \quad \theta_{0}^{\delta, \eta} \triangleq \frac{j_{\delta} * \theta_{0}+\eta}{1+\eta}
$$

where $j_{\delta}$ is the standard mollifying kernel of width $\delta$. Then, $\left(\rho_{0}^{\delta, \eta}, u_{0}^{\delta, \eta}, \theta_{0}^{\delta, \eta}\right)$ satisfies

$$
\left\{\begin{array}{l}
\left(\rho_{0}^{\delta, \eta}-1, u_{0}^{\delta, \eta}, \theta_{0}^{\delta, \eta}-1\right) \in H^{\infty} \\
\frac{\eta}{1+\eta} \leq \rho_{0}^{\delta, \eta} \leq \frac{\bar{\rho}+\eta}{1+\eta}<\bar{\rho}, \quad \theta_{0}^{\delta, \eta} \geq \frac{\eta}{\bar{\rho}+\eta}, \quad\left\|\nabla u_{0}^{\delta, \eta}\right\|_{L^{2}} \leq M,
\end{array}\right.
$$

and

$$
\left\{\begin{array}{l}
\lim _{\delta+\eta \rightarrow 0}\left(\left\|\rho_{0}^{\delta, \eta}-\rho_{0}\right\|_{H^{2} \cap W^{2, q}}+\left\|u_{0}^{\delta, \eta}-u_{0}\right\|_{H^{2}}+\left\|\theta_{0}^{\delta, \eta}-\theta_{0}\right\|_{H^{2}}\right)=0, \\
\left\|\nabla\left(\rho_{0}^{\delta, \eta}, u_{0}^{\delta, \eta}, \theta_{0}^{\delta, \eta}\right)\right\|_{H^{1}} \leq\left\|\nabla\left(\rho_{0}, u_{0}, \theta_{0}\right)\right\|_{H^{1}}, \quad\left\|\nabla \rho_{0}^{\delta, \eta}\right\|_{W^{1, q}} \leq\left\|\nabla \rho_{0}\right\|_{W^{1, q}},
\end{array}\right.
$$

due to (1.9) and (1.10). Moreover, the initial norm $C_{0}^{\delta, \eta}$ for $\left(\rho_{0}^{\delta, \eta}, u_{0}^{\delta, \eta}, \theta_{0}^{\delta, \eta}\right)$, i.e., the right hand side of (1.8) with $\left(\rho_{0}, u_{0}, \theta_{0}\right)$ replaced by $\left(\rho_{0}^{\delta, \eta}, u_{0}^{\delta, \eta}, \theta_{0}^{\delta, \eta}\right)$, satisfies

$$
\lim _{\eta \rightarrow 0} \lim _{\delta \rightarrow 0} C_{0}^{\delta, \eta}=C_{0} .
$$

Therefore, there exists an $\eta_{0} \in\left(0, \min \left\{1, \bar{\rho}-\sup _{x \in \mathbb{R}^{3}} \rho_{0}(x)\right\}\right)$ such that, for any $\eta \in\left(0, \eta_{0}\right)$, we can find some $\delta_{0}(\eta)>0$ such that

$$
C_{0}^{\delta, \eta} \leq C_{0}+\varepsilon_{0} / 2 \leq \varepsilon_{0}
$$

provided that

$$
0<\eta \leq \eta_{0}, \quad 0<\delta \leq \delta_{0}(\eta) .
$$

We assume that $\delta, \eta$ satisfy (5.18). Proposition 5.1 together with (5.17) and (5.15) thus yields that there exists a smooth solution $\left(\rho^{\delta, \eta}, u^{\delta, \eta}, \theta^{\delta, \eta}\right)$ of (1.6) (1.4) (1.7) with initial data $\left(\rho_{0}^{\delta, \eta}, u_{0}^{\delta, \eta}, \theta_{0}^{\delta, \eta}\right)$ on $\mathbb{R}^{3} \times[0, T]$ for all $T>0$. Moreover, (3.9) and (3.6) both hold with $(\rho, u, \theta)$ being replaced by $\left(\rho^{\delta, \eta}, u^{\delta, \eta}, \theta^{\delta, \eta}\right)$.

Next, for the initial data $\left(\rho_{0}^{\delta, \eta}, u_{0}^{\delta, \eta}, \theta_{0}^{\delta, \eta}\right)$, the $\tilde{g_{1}}$ in (4.1) in fact is

$$
\begin{aligned}
\tilde{g}_{1} \triangleq & \left(\rho_{0}^{\delta, \eta}\right)^{-1 / 2}\left(-\mu \Delta u_{0}^{\delta, \eta}-(\mu+\lambda) \nabla \operatorname{div} u_{0}^{\delta, \eta}+R \nabla\left(\rho_{0}^{\delta, \eta} \theta_{0}^{\delta, \eta}\right)\right) \\
= & \left(\rho_{0}^{\delta, \eta}\right)^{-1 / 2}\left(j_{\delta} * \rho_{0}\right)^{1 / 2} g_{1}+\left(\rho_{0}^{\delta, \eta}\right)^{-1 / 2}\left(j_{\delta} *\left(\sqrt{\rho_{0}} g_{1}\right)-\sqrt{j_{\delta} * \rho_{0}} g_{1}\right) \\
& +R\left(\rho_{0}^{\delta, \eta}\right)^{-1 / 2} \nabla\left(j_{\delta} *\left(\rho_{0} \theta_{0}\right)-(1+\eta)^{-2}\left(j_{\delta} * \rho_{0}\right)\left(j_{\delta} * \theta_{0}\right)\right) \\
& +R \eta(1+\eta)^{-2}\left(\rho_{0}^{\delta, \eta}\right)^{-1 / 2} \nabla\left(\rho_{0}^{\delta, \eta}+\theta_{0}^{\delta, \eta}\right),
\end{aligned}
$$


where in the second equality we have used (1.11). Similarly, the $\tilde{g_{2}}$ in (4.2) is

$$
\begin{aligned}
\tilde{g}_{2} \triangleq & \left(\rho_{0}^{\delta, \eta}\right)^{-1 / 2}\left(\kappa \Delta \theta_{0}^{\delta, \eta}+\frac{\mu}{2}\left|\nabla u_{0}^{\delta, \eta}+\left(\nabla u_{0}^{\delta, \eta}\right)^{\operatorname{tr}}\right|^{2}+\lambda\left(\operatorname{div} u_{0}^{\delta, \eta}\right)^{2}\right) \\
= & \left(\rho_{0}^{\delta, \eta}\right)^{-1 / 2}\left(j_{\delta} * \rho_{0}\right)^{1 / 2} g_{2}+\left(\rho_{0}^{\delta, \eta}\right)^{-1 / 2}\left(j_{\delta} *\left(\sqrt{\rho_{0}} g_{2}\right)-\sqrt{j_{\delta} * \rho_{0}} g_{2}\right) \\
& -\frac{\mu}{2}\left(\rho_{0}^{\delta, \eta}\right)^{-1 / 2}\left(j_{\delta} *\left(\left|\nabla u_{0}+\left(\nabla u_{0}\right)^{\operatorname{tr}}\right|^{2}\right)-\left|\nabla\left(j_{\delta} * u_{0}\right)+\left(\nabla\left(j_{\delta} * u_{0}\right)\right)^{\operatorname{tr}}\right|^{2}\right) \\
& -\lambda\left(\rho_{0}^{\delta, \eta}\right)^{-1 / 2}\left(j_{\delta} *\left(\left(\operatorname{div} u_{0}\right)^{2}\right)-\left(\operatorname{div}\left(j_{\delta} * u_{0}\right)\right)^{2}\right),
\end{aligned}
$$

due to (1.12). Since $g_{1}, g_{2} \in L^{2}$, one deduces from (5.19), (5.20), (5.15), (5.16), and (1.9) that there exists some positive constant $C$ independent of $\delta$ and $\eta$ such that

$$
\left\{\begin{array}{l}
\left\|\tilde{g}_{1}\right\|_{L^{2}} \leq(1+\eta)^{1 / 2}\left\|g_{1}\right\|_{L^{2}}+C \eta^{-1 / 2} m_{1}(\delta)+C \sqrt{\eta}, \\
\left\|\tilde{g}_{2}\right\|_{L^{2}} \leq(1+\eta)^{1 / 2}\left\|g_{2}\right\|_{L^{2}}+C \eta^{-1 / 2} m_{2}(\delta),
\end{array}\right.
$$

with $0 \leq m_{i}(\delta) \rightarrow 0(i=1,2)$ as $\delta \rightarrow 0$. Hence, for any $0<\eta<\eta_{0}$, there exists some $0<\delta_{1}(\eta) \leq \delta_{0}(\eta)$ such that

$$
m_{1}(\delta)+m_{2}(\delta)<\eta,
$$

for any $0<\delta<\delta_{1}(\eta)$. We thus obtain from (5.21) and (5.22) that there exists some positive constant $C$ independent of $\delta$ and $\eta$ such that

$$
\left\|\tilde{g}_{1}\right\|_{L^{2}}+\left\|\tilde{g}_{2}\right\|_{L^{2}} \leq 2\left\|g_{1}\right\|_{L^{2}}+2\left\|g_{2}\right\|_{L^{2}}+C
$$

provided that

$$
0<\eta<\eta_{0}, \quad 0<\delta<\delta_{1}(\eta) .
$$

Now, we assume that $\eta, \delta$ satisfy (5.24). It thus follows from (5.17), Proposition 3.1. Corollary [3.9, (5.16), (5.23), and Lemmas 4.144.6 that for any $T>0$, there exists some positive constant $C$ independent of $\delta$ and $\eta$ such that (3.9), (3.6), (3.102), (4.22), (4.23), (4.52), (4.62), and (4.80) hold for $\left(\rho^{\delta, \eta}, u^{\delta, \eta}, \theta^{\delta, \eta}\right)$. Then passing to the limit first $\delta \rightarrow 0$, then $\eta \rightarrow 0$, together with standard arguments yields that there exists a solution $(\rho, u, \theta)$ of (1.6) (1.4) (1.7) on $\mathbb{R}^{3} \times(0, T]$ for all $T>0$, such that $(\rho, u, \theta)$ satisfies (3.9), (3.6), (3.102), (4.22), (4.23), (4.52), (4.62) and (4.80). Hence, $(\rho, u, \theta)$ satisfies (1.14), $(1.15)_{2},(1.15)_{3}$, and

$$
\rho-1 \in L^{\infty}\left(0, T ; H^{2} \cap W^{2, q}\right), \quad(u, \theta-1) \in L^{\infty}\left(0, T ; H^{2}\right) .
$$

Moreover, (4.58) holds in $\mathcal{D}^{\prime}\left(\mathbb{R}^{3} \times(0, T)\right)$.

Next, to finish the existence part of Theorem 1.1, it remains to prove

$$
\rho-1 \in C\left([0, T] ; H^{2} \cap W^{2, q}\right), \quad u, \theta-1 \in C\left([0, T] ; H^{2}\right) .
$$

It follows from (4.22) and (5.25) that

$$
\rho-1 \in C\left([0, T] ; H^{1} \cap W^{1, \infty}\right) \cap C\left([0, T] ; H^{2} \cap W^{2, q} \text {-weak }\right),
$$

and for all $r \in[2,6)$,

$$
u, \theta-1 \in C\left([0, T] ; H^{1} \cap W^{1, r}\right) .
$$

Since (4.58) holds in $\mathcal{D}^{\prime}\left(\mathbb{R}^{3} \times(0, T)\right)$ for all $T \in(0, \infty)$, one derives from [16, Lemma $2.3]$ that, for $j_{\nu}(x)$ being the standard mollifying kernel of width $\nu, \rho^{\nu} \triangleq \rho * j_{\nu}$ satisfies

$$
\left(\Delta \rho^{\nu}\right)_{t}+\operatorname{div}\left(u \Delta \rho^{\nu}\right)=-\operatorname{div}(\rho \Delta u) * j_{\nu}-2 \operatorname{div}\left(\partial_{i} \rho \cdot \partial_{i} u\right) * j_{\nu}+R_{\nu}
$$


where $R_{\nu}$ satisfies

$$
\int_{0}^{T}\left\|R_{\nu}\right\|_{L^{2} \cap L^{q}}^{3 / 2} d t \leq C \int_{0}^{T}\|u\|_{W^{1, \infty}}^{3 / 2}\|\Delta \rho\|_{L^{2} \cap L^{q}}^{3 / 2} d t \leq C,
$$

due to (4.5), (4.22), and (4.52). Multiplying (5.29) by $q\left|\Delta \rho^{\nu}\right|^{q-2} \Delta \rho^{\nu}$, we obtain after integration by parts that

$$
\begin{aligned}
& \left(\left\|\Delta \rho^{\nu}\right\|_{L^{q}}^{q}\right)^{\prime}(t) \\
& =(1-q) \int\left|\Delta \rho^{\nu}\right|^{q} \operatorname{div} u d x-q \int\left(\operatorname{div}(\rho \Delta u) * j_{\nu}\right)\left|\Delta \rho^{\nu}\right|^{q-2} \Delta \rho^{\nu} d x \\
& \quad-2 q \int\left(\operatorname{div}\left(\partial_{i} \rho \cdot \partial_{i} u\right) * j_{\nu}\right)\left|\Delta \rho^{\nu}\right|^{q-2} \Delta \rho^{\nu} d x+q \int R_{\nu}\left|\Delta \rho^{\nu}\right|^{q-2} \Delta \rho^{\nu} d x,
\end{aligned}
$$

which together with (4.22), (4.52), and (5.30) yields that, for $p_{0}$ as in (4.53),

$$
\begin{aligned}
& \sup _{t \in[0, T]}\left\|\Delta \rho^{\nu}\right\|_{L^{q}}+\int_{0}^{T}\left|\left(\left\|\Delta \rho^{\nu}\right\|_{L^{q}}^{q}\right)^{\prime}(t)\right|^{p_{0}} d t \\
& \leq C+C \int_{0}^{T}\left(\|\nabla u\|_{W^{2, q}}^{p_{0}}+\left\|R_{\nu}\right\|_{L^{2} \cap L^{q}}^{p_{0}}\right) d t \\
& \leq C .
\end{aligned}
$$

This fact combining with the Ascoli-Arzela theorem thus leads to

$$
\left\|\Delta \rho^{\nu}(\cdot, t)\right\|_{L^{q}} \rightarrow\|\Delta \rho(\cdot, t)\|_{L^{q}} \text { in } C([0, T]), \text { as } \nu \rightarrow 0^{+} .
$$

In particular, we have

$$
\left\|\nabla^{2} \rho(\cdot, t)\right\|_{L^{q}} \in C([0, T]) .
$$

Similarly, one can obtain that

$$
\left\|\nabla^{2} \rho(\cdot, t)\right\|_{L^{2}} \in C([0, T]) .
$$

Therefore, the continuity of $\nabla^{2} \rho$ in $L^{p}(p=2, q)$, i.e.,

$$
\nabla^{2} \rho \in C\left([0, T] ; L^{2} \cap L^{q}\right),
$$

follows directly from (5.27), (5.31), and (5.32).

It follows from (4.22) and (4.23) that

$$
\rho u_{t}, \rho \theta_{t} \in C\left([0, T] ; L^{2}\right),
$$

which together with (4.13), (5.27), (5.28), and (5.33) gives

$$
u \in C\left([0, T] ; H^{2}\right) .
$$

This fact combining with (3.40), (5.34), (5.33), (5.28), and (4.22) leads to

$$
\theta-1 \in C\left([0, T] ; H^{2}\right),
$$

which as well as (5.27), (5.33), and (5.35) leads to (5.26).

Finally, since the proof of the uniqueness of $(\rho, u, \theta)$ is similar to that of [4, Theorem 1], to finish the proof of Theorem 1.1, it remains to prove (1.16). We will only show

$$
\lim _{t \rightarrow \infty}\|\nabla u\|_{L^{2}}=0
$$


since the other terms in (1.16) follow directly from (1.27). It follows from (3.102) and (3.6) that

$$
\begin{aligned}
& \int_{1}^{\infty}\left|\left(\|\nabla u\|_{L^{2}}^{2}\right)^{\prime}(t)\right| d t \\
& =2 \int_{1}^{\infty}\left|\int \partial_{j} u^{i} \partial_{j} u_{t}^{i} d x\right| d t \\
& =2 \int_{1}^{\infty}\left|\int \partial_{j} u^{i} \partial_{j}\left(\dot{u}^{i}-u^{k} \partial_{k} u^{i}\right) d x\right| d t \\
& =2 \int_{1}^{\infty}\left|\int\left(\partial_{j} u^{i} \partial_{j} \dot{u}^{i}-\partial_{j} u^{i} \partial_{j} u^{k} \partial_{k} u^{i}-\partial_{j} u^{i} u^{k} \partial_{k j} u^{i}\right) d x\right| d t \\
& =\int_{1}^{\infty}\left|\int\left(2 \partial_{j} u^{i} \partial_{j} \dot{u}^{i}-2 \partial_{j} u^{i} \partial_{j} u^{k} \partial_{k} u^{i}+|\nabla u|^{2} \operatorname{div} u\right) d x\right| d t \\
& \leq C \int_{1}^{\infty}\left(\|\nabla u\|_{L^{2}}\|\nabla \dot{u}\|_{L^{2}}+\|\nabla u\|_{L^{3}}^{3}\right) d t \\
& \leq C \int_{1}^{\infty}\left(\|\nabla \dot{u}\|_{L^{2}}^{2}+\|\nabla u\|_{L^{2}}^{2}+\|\nabla u\|_{L^{4}}^{4}\right) d t \\
& \leq C
\end{aligned}
$$

which together with (3.6) implies (5.36). We finish the proof of Theorem 1.1

Proof of Theorem 1.2. We will prove Theorem 1.2 in three steps.

Step 1. Construction of approximate solutions. Let $\left(\rho_{0}, u_{0}, \theta_{0}\right)$ satisfying (1.10) be initial data as described in Theorem 1.2. Assume that $C_{0}$ satisfies $(1.22)$ with $\varepsilon$ as in (5.12). Let $\delta$ and $\eta$ be as in (5.13) and $j_{\delta}$ be the standard mollifier. We define

$$
\hat{\rho}_{0}^{\delta, \eta} \triangleq \frac{j_{\delta} * \rho_{0}+\eta}{1+\eta}, \quad \hat{u}_{0}^{\delta, \eta} \triangleq j_{\delta} * u_{0}, \quad \hat{\theta}_{0}^{\delta, \eta} \triangleq \frac{j_{\delta} *\left(\rho_{0} \theta_{0}\right)+\eta}{j_{\delta} * \rho_{0}+\eta} .
$$

Then, $\left(\hat{\rho}_{0}^{\delta, \eta}, \hat{u}_{0}^{\delta, \eta}, \hat{\theta}_{0}^{\delta, \eta}\right)$ satisfies

$$
\left\{\begin{array}{l}
\left(\hat{\rho}_{0}^{\delta, \eta}-1, \hat{u}_{0}^{\delta, \eta}, \hat{\theta}_{0}^{\delta, \eta}-1\right) \in H^{\infty} \\
0<\frac{\eta}{1+\eta} \leq \hat{\rho}_{0}^{\delta, \eta} \leq \frac{\bar{\rho}+\eta}{1+\eta}<\bar{\rho}, \quad \hat{\theta}_{0}^{\delta, \eta} \geq \frac{\eta}{\bar{\rho}+\eta}>0, \quad\left\|\nabla \hat{u}_{0}^{\delta, \eta}\right\|_{L^{2}} \leq M
\end{array}\right.
$$

due to (1.10). Moreover, it follows from (1.10) and (1.22) that

$$
\lim _{\eta \rightarrow 0} \lim _{\delta \rightarrow 0}\left(\left\|\hat{\rho}_{0}^{\delta, \eta}-\rho_{0}\right\|_{L^{2}}+\left\|\hat{u}_{0}^{\delta, \eta}-u_{0}\right\|_{H^{1}}+\left\|\hat{\rho}_{0}^{\delta, \eta} \hat{\theta}_{0}^{\delta, \eta}-\rho_{0} \theta_{0}\right\|_{L^{2}}\right)=0 .
$$

We claim that the initial norm $\hat{C}_{0}^{\delta, \eta}$ for $\left(\hat{\rho}_{0}^{\delta, \eta}, \hat{u}_{0}^{\delta, \eta}, \hat{\theta}_{0}^{\delta, \eta}\right)$, i.e., the right hand side of (1.8) with $\left(\rho_{0}, u_{0}, \theta_{0}\right)$ replaced by $\left(\hat{\rho}_{0}^{\delta, \eta}, \hat{u}_{0}^{\delta, \eta}, \hat{\theta}_{0}^{\delta, \eta}\right)$, satisfies

$$
\lim _{\eta \rightarrow 0} \lim _{\delta \rightarrow 0} \hat{C}_{0}^{\delta, \eta} \leq C_{0}
$$

which yields that there exists an $\hat{\eta}>0$ such that, for any $\eta \in(0, \hat{\eta})$, there exists some $\hat{\delta}(\eta)>0$ such that

$$
\hat{C}_{0}^{\delta, \eta} \leq C_{0}+\varepsilon_{0} / 2 \leq \varepsilon_{0}
$$

provided

$$
0<\eta \leq \hat{\eta}, \quad 0<\delta \leq \hat{\delta}(\eta)
$$


We assume that $\delta, \eta$ always satisfy (5.42). Proposition 5.1 as well as (5.38) and (5.41) thus yields that there exists a smooth solution $\left(\hat{\rho}^{\delta, \eta}, \hat{u}^{\delta, \eta}, \hat{\theta}^{\delta, \eta}\right)$ of (1.6) (1.4) (1.7) with initial data $\left(\hat{\rho}_{0}^{\delta, \eta}, \hat{u}_{0}^{\delta, \eta}, \hat{\theta}_{0}^{\delta, \eta}\right)$ on $\mathbb{R}^{3} \times[0, T]$ for all $T>0$. Moreover, for any $T>0$, $\left(\hat{\rho}^{\delta, \eta}, \hat{u}^{\delta, \eta}, \hat{\theta}^{\delta, \eta}\right)$ satisfies (3.9) ( (3.6)), and (3.102) with $(\rho, u, \theta)$ replaced by $\left(\hat{\rho}^{\delta, \eta}, \hat{u}^{\delta, \eta}, \hat{\theta}^{\delta, \eta}\right)$.

It remains to prove (5.40). In fact, we only have to show

$$
\lim _{\eta \rightarrow 0} \lim _{\delta \rightarrow 0} \int \hat{\rho}_{0}^{\delta, \eta}\left(\hat{\theta}_{0}^{\delta, \eta}-\log \hat{\theta}_{0}^{\delta, \eta}-1\right) d x \leq \int \rho_{0}\left(\theta_{0}-\log \theta_{0}-1\right) d x,
$$

since the other terms in (5.40) can be proved in a similar and even simpler way. Noticing that

$$
\begin{aligned}
& \hat{\rho}_{0}^{\delta, \eta}\left(\hat{\theta}_{0}^{\delta, \eta}-\log \hat{\theta}_{0}^{\delta, \eta}-1\right) \\
& =\hat{\rho}_{0}^{\delta, \eta}\left(\hat{\theta}_{0}^{\delta, \eta}-1\right)^{2} \int_{0}^{1} \frac{\alpha}{\alpha\left(\hat{\theta}_{0}^{\delta, \eta}-1\right)+1} d \alpha \\
& =\frac{\left(j_{\delta} *\left(\rho_{0} \theta_{0}-\rho_{0}\right)\right)^{2}}{1+\eta} \int_{0}^{1} \frac{\alpha}{\alpha\left(j_{\delta} *\left(\rho_{0} \theta_{0}\right)-j_{\delta} * \rho_{0}\right)+j_{\delta} * \rho_{0}+\eta} d \alpha \\
& \in\left[0, \eta^{-1}\left(j_{\delta} *\left(\rho_{0} \theta_{0}-\rho_{0}\right)\right)^{2}\right]
\end{aligned}
$$

we deduce from (5.39) and Lebesgue's dominated convergence theorem that

$$
\begin{aligned}
& \lim _{\delta \rightarrow 0} \int \hat{\rho}_{0}^{\delta, \eta}\left(\hat{\theta}_{0}^{\delta, \eta}-\log \hat{\theta}_{0}^{\delta, \eta}-1\right) d x \\
&=\int \frac{\rho_{0}+\eta}{1+\eta}\left(\frac{\rho_{0} \theta_{0}+\eta}{\rho_{0}+\eta}-\log \frac{\rho_{0} \theta_{0}+\eta}{\rho_{0}+\eta}-1\right) d x \\
&=(1+\eta)^{-1} \int_{\left(\rho_{0} \theta_{0}<1 / 2\right) \cup\left(\rho_{0} \theta_{0}>2\right)}\left(\rho_{0} \theta_{0}-\rho_{0}-\left(\rho_{0}+\eta\right) \log \frac{\rho_{0} \theta_{0}+\eta}{\rho_{0}+\eta}\right) d x \\
& \quad+(1+\eta)^{-1} \int_{\left(1 / 2 \leq \rho_{0} \theta_{0} \leq 2\right)}\left(\rho_{0}+\eta\right)\left(\frac{\rho_{0} \theta_{0}+\eta}{\rho_{0}+\eta}-\log \frac{\rho_{0} \theta_{0}+\eta}{\rho_{0}+\eta}-1\right) d x \\
& \triangleq(1+\eta)^{-1} I_{1}+(1+\eta)^{-1} I_{2},
\end{aligned}
$$

where we have used the following simple fact that, for $f \in L^{p}(1 \leq p<\infty)$,

$$
\lim _{\delta \rightarrow 0}\left\|j_{\delta} * f-f\right\|_{L^{p}}=0, \quad \lim _{\delta \rightarrow 0} j_{\delta} * f(x)=f(x), \text { a.e. } x \in \mathbb{R}^{3} .
$$

It follows from (1.22) that

$$
\begin{aligned}
& \left|\left(\rho_{0} \theta_{0}<1 / 2\right) \cup\left(\rho_{0} \theta_{0}>2\right)\right| \\
& \leq 4 \int\left(\rho_{0} \theta_{0}-1\right)^{2} d x \\
& \leq 8 \int\left(\rho_{0} \theta_{0}-\rho_{0}\right)^{2} d x+8 \int\left(\rho_{0}-1\right)^{2} d x \\
& \leq C,
\end{aligned}
$$


which combining with Lebesgue's dominated convergence theorem yields

$$
\begin{aligned}
I_{1}= & \int_{\left(\rho_{0} \theta_{0}<1 / 2\right) \cup\left(\rho_{0} \theta_{0}>2\right)}\left(\rho_{0} \theta_{0}-\rho_{0} \log \left(\rho_{0} \theta_{0}+\eta\right)-\eta \log \left(\rho_{0} \theta_{0}+\eta\right)\right) d x \\
& +\int_{\left(\rho_{0} \theta_{0}<1 / 2\right) \cup\left(\rho_{0} \theta_{0}>2\right)}\left(\left(\rho_{0}+\eta\right) \log \left(\rho_{0}+\eta\right)-\rho_{0}\right) d x \\
\leq & \int_{\left(\rho_{0} \theta_{0}<1 / 2\right) \cup\left(\rho_{0} \theta_{0}>2\right)}\left(\rho_{0} \theta_{0}-\rho_{0} \log \left(\rho_{0} \theta_{0}\right)-\eta \log \eta\right) d x \\
& +\int_{\left(\rho_{0} \theta_{0}<1 / 2\right) \cup\left(\rho_{0} \theta_{0}>2\right)}\left(\rho_{0} \log \left(\rho_{0}+\eta\right)+\eta \log \left(\rho_{0}+\eta\right)-\rho_{0}\right) d x \\
\rightarrow & \int_{\left(\rho_{0} \theta_{0}<1 / 2\right) \cup\left(\rho_{0} \theta_{0}>2\right)} \rho_{0}\left(\theta_{0}-\log \theta_{0}-1\right) d x, \quad \text { as } \eta \rightarrow 0 .
\end{aligned}
$$

Noticing that

$$
\begin{aligned}
& \left(\rho_{0}+\eta\right)\left(\frac{\rho_{0} \theta_{0}+\eta}{\rho_{0}+\eta}-\log \frac{\rho_{0} \theta_{0}+\eta}{\rho_{0}+\eta}-1\right) \\
& =\left(\rho_{0} \theta_{0}-\rho_{0}\right)^{2} \int_{0}^{1} \frac{\alpha}{\alpha\left(\rho_{0} \theta_{0}-\rho_{0}\right)+\rho_{0}+\eta} d \alpha \\
& \in\left[0,2\left(\rho_{0} \theta_{0}-\rho_{0}\right)^{2}\right],
\end{aligned}
$$

provided $\rho_{0} \theta_{0} \geq 1 / 2$, we deduce from Lebesgue's dominated convergence theorem that

$$
\lim _{\eta \rightarrow 0} I_{2}=\int_{\left(1 / 2 \leq \rho_{0} \theta_{0} \leq 2\right)} \rho_{0}\left(\theta_{0}-\log \theta_{0}-1\right) d x,
$$

which together with (5.44) and (5.45) gives (5.43).

Step 2. Compactness results. For the approximate solutions $\left(\hat{\rho}^{\delta, \eta}, \hat{u}^{\delta, \eta}, \hat{\theta}^{\delta, \eta}\right)$ obtained in the previous step, we will pass to the limit first $\delta \rightarrow 0$, then $\eta \rightarrow 0$ and apply (3.6) and (3.102) to obtain the global existence of weak solutions. Since the two steps are similar, we will only sketch the arguments for $\delta \rightarrow 0$. Thus, we fix $\eta \in(0, \hat{\eta})$ and simply denote $\left(\hat{\rho}^{\delta, \eta}, \hat{u}^{\delta, \eta}, \hat{\theta}^{\delta, \eta}\right)$ by $\left(\rho^{\delta}, u^{\delta}, \theta^{\delta}\right)$. For $R \in(0, \infty)$, let $B_{R}\left(x_{0}\right) \triangleq\left\{x \in \mathbb{R}^{3}|| x-x_{0} \mid<R\right\}$ denote a ball centered at $x_{0} \in \mathbb{R}^{3}$ with radius $R$. We claim that there exists some appropriate subsequence $\delta_{j} \rightarrow 0$ of $\delta \rightarrow 0$ such that, for any $0<\tau<T<\infty$ and $0<R<\infty$, we have

$$
\begin{gathered}
\left\{\begin{array}{l}
\theta^{\delta_{j}}-1 \rightarrow \theta-1 \text { weakly in } L^{2}\left(0, T ; H^{1}\left(\mathbb{R}^{3}\right)\right), \\
u^{\delta_{j}}-u \text { weakly star in } L^{\infty}\left(0, T ; H^{1}\left(\mathbb{R}^{3}\right)\right),
\end{array}\right. \\
\left\{\begin{array}{l}
\rho^{\delta_{j}}-1 \rightarrow \rho-1 \quad \text { in } C\left([0, T] ; L^{2}\left(\mathbb{R}^{3}\right) \text {-weak }\right), \\
\rho^{\delta_{j}}-1 \rightarrow \rho-1 \quad \text { in } C\left([0, T] ; H^{-1}\left(B_{R}(0)\right)\right),
\end{array}\right. \\
\left\{\begin{array}{l}
\rho^{\delta_{j}} u^{\delta_{j}} \rightarrow \rho u, \rho^{\delta_{j}}\left(\theta^{\delta_{j}}-1\right) \rightarrow \rho(\theta-1) \quad \text { in } C\left([0, T] ; L^{2}\left(\mathbb{R}^{3}\right) \text {-weak }\right), \\
\rho^{\delta_{j}} u^{\delta_{j}} \rightarrow \rho u \text { in } C\left([0, T] ; H^{-1}\left(B_{R}(0)\right)\right),
\end{array}\right. \\
\rho^{\delta_{j}}\left|u^{\delta_{j}}\right|^{2} \rightarrow \rho|u|^{2} \text { in } C\left([0, T] ; L^{3} \text {-weak }\right),
\end{gathered}
$$

and

$$
\left\{\begin{array}{l}
u^{\delta_{j}} \rightarrow u, G^{\delta_{j}} \rightarrow G, \omega^{\delta_{j}} \rightarrow \omega, \nabla \theta^{\delta_{j}} \rightarrow \nabla \theta \text { in } C\left([\tau, T] ; H^{1}\left(\mathbb{R}^{3}\right) \text {-weak }\right), \\
u^{\delta_{j}} \rightarrow u, G^{\delta_{j}} \rightarrow G, \omega^{\delta_{j}} \rightarrow \omega, \nabla \theta^{\delta_{j}} \rightarrow \nabla \theta \text { in } C\left([\tau, T] ; L^{2}\left(B_{R}(0)\right)\right) .
\end{array}\right.
$$


We thus write (1.1) in the weak forms for the approximate solutions $\left(\rho^{\delta}, u^{\delta}, \theta^{\delta}\right)$, then let $\delta=\delta_{j}$ and take appropriate limits. Standard arguments as well as (5.46)-(5.50) thus yield that the limit $(\rho, u, \theta)$ is a weak solution of (1.1) (1.4) (1.5) in the sense of Definition 1.1 and satisfies (1.23)-(1.26) except $\rho-1 \in C\left([0, \infty), L^{2}\right)$ which in fact can be obtained by similar arguments leading to (5.33). In addition, the estimates (1.28) -(1.30) follows direct from (3.9), (3.102), (3.6), and (5.46)-(5.50).

It remains to prove (5.47)-(5.50) since (5.46) is a direct consequence of (3.6). It follows from (3.9), (3.6), and (1.6) $)_{1}$ that

$$
\sup _{t \in[0, \infty)}\left\|\rho_{t}^{\delta}\right\|_{H^{-1}\left(\mathbb{R}^{3}\right)} \leq C,
$$

which as well as (3.6), [16, Lemma C.1], and the Aubin-Lions lemma yields that there exists a subsequence of $\delta_{j} \rightarrow 0$, still denoted by $\delta_{j}$, such that (5.47) holds. Moreover, one deduces from (3.102) that (extract a subsequence)

$$
\rho^{\delta_{j}}-1 \rightarrow \rho-1, \nabla u^{\delta_{j}} \rightarrow \nabla u \text { weakly in } L^{4}\left(\mathbb{R}^{3} \times(1, \infty)\right),
$$

with $\rho-1$ and $\nabla u$ satisfying

$$
\int_{1}^{\infty}\left(\|\rho-1\|_{L^{4}}^{4}+\|\nabla u\|_{L^{4}}^{4}\right) d t \leq C .
$$

Then, simple calculations together with (3.6) yield that, for any $0<T<\infty$, there exists some $C(T)$ independent of $\delta$ and $\eta$ such that

$$
\left\|\left(\rho^{\delta} u^{\delta}\right)_{t}\right\|_{L^{2}\left(0, T ; H^{-1}\left(\mathbb{R}^{3}\right)\right)}+\left\|\left(\rho^{\delta} \theta^{\delta}\right)_{t}\right\|_{L^{2}\left(0, T ; H^{-1}\left(\mathbb{R}^{3}\right)\right)} \leq C(T)
$$

which together with (3.6), (5.47), and (5.46) gives (5.48).

Next, to prove (5.49), one deduces from (3.6) and (1.6) $)_{1}$ that, for any $\zeta \in H^{1}\left(\mathbb{R}^{3}\right)$,

$$
\begin{aligned}
& \left|\int\left(\rho^{\delta}\left|u^{\delta}\right|^{2}\right)_{t} \zeta d x\right| \\
& =\left.\left|-\int \operatorname{div}\left(\rho^{\delta} u^{\delta}\right)\right| u^{\delta}\right|^{2} \zeta d x+2 \int \rho^{\delta} u^{\delta} \cdot u_{t}^{\delta} \zeta d x \mid \\
& =\left|\int \rho^{\delta} u^{\delta} \cdot \nabla\left(\left|u^{\delta}\right|^{2} \zeta\right) d x+2 \int \rho^{\delta} u^{\delta} \cdot\left(\dot{u}^{\delta}-u^{\delta} \cdot \nabla u^{\delta}\right) \zeta d x\right| \\
& \leq C \int \rho^{\delta}\left|u^{\delta}\right|^{3}|\nabla \zeta| d x+C \int \rho^{\delta}\left|u^{\delta}\right|^{2}\left|\nabla u^{\delta}\left\|\zeta\left|d x+C \int \rho^{\delta}\right| u^{\delta}\right\| \dot{u}^{\delta} \| \zeta\right| d x \\
& \leq C\left\|u^{\delta}\right\|_{L^{6}}^{3}\|\nabla \zeta\|_{L^{2}}+C\left\|u^{\delta}\right\|_{L^{6}}^{2}\left\|\nabla u^{\delta}\right\|_{L^{2}}\|\zeta\|_{L^{6}}+C\left\|u^{\delta}\right\|_{L^{6}}\left\|\left(\rho^{\delta}\right)^{1 / 2} \dot{u}^{\delta}\right\|_{L^{2}}\|\zeta\|_{L^{3}} \\
& \leq C\left(\left\|\nabla u^{\delta}\right\|_{L^{2}}+\left\|\left(\rho^{\delta}\right)^{1 / 2} \dot{u}^{\delta}\right\|_{L^{2}}\right)\|\zeta\|_{H^{1}},
\end{aligned}
$$

which together with (3.6) gives

$$
\int_{0}^{\infty}\left\|\left(\rho^{\delta}\left|u^{\delta}\right|^{2}\right)_{t}\right\|_{H^{-1}}^{2} d t \leq C .
$$

It follows from (3.6) that

$$
\sup _{t \in[0, \infty)}\left\|\rho^{\delta}\left|u^{\delta}\right|^{2}\right\|_{L^{1} \cap L^{3}} \leq C,
$$


which combining with (5.53), (5.46), and (5.48) yields (5.49).

Finally, we prove (5.50) which implies the strong limits of $u^{\delta}$ and $\theta^{\delta}$. We deduce from (3.6), (2.27), (15.52), and (3.102) that

$$
\sup _{t \in[0, \infty)}\left(\left\|u^{\delta}\right\|_{H^{1}}+\sigma^{2}\left\|G^{\delta}\right\|_{H^{1}}+\sigma^{2}\left\|\omega^{\delta}\right\|_{H^{1}}+\sigma^{2}\left\|\nabla \theta^{\delta}\right\|_{H^{1}}\right) \leq C
$$

and

$$
\int_{\tau}^{T}\left(\left\|u_{t}^{\delta}\right\|_{L^{2}\left(\mathbb{R}^{3}\right)}^{2}+\left\|G_{t}^{\delta}\right\|_{H^{-1}\left(\mathbb{R}^{3}\right)}^{2}+\left\|\omega_{t}^{\delta}\right\|_{H^{-1}\left(\mathbb{R}^{3}\right)}^{2}+\left\|\theta_{t}^{\delta}\right\|_{H^{1}\left(\mathbb{R}^{3}\right)}^{2}\right) d t \leq C(\tau, T),
$$

for all $0<\tau<T<\infty$. The Aubin-Lions lemma together with (5.54) and (5.55) thus gives (5.50).

Step 3. Proofs of (1.31) and (1.27). We first prove that $(\rho, u, \theta)$ satisfies (1.31). We rewrite the energy equation $(1.6)_{3}$ in the form

$$
\begin{aligned}
& \frac{R}{\gamma-1}\left((\rho \theta)_{t}+\operatorname{div}(\rho u \theta)\right)-\kappa \Delta \theta \\
& =G \operatorname{div} u-R \operatorname{div} u+2 \mu \operatorname{div}(u \cdot \nabla u-u \operatorname{div} u)+\frac{\mu}{2}|\omega|^{2} .
\end{aligned}
$$

Thus, for any $\varphi \in \mathcal{D}\left(\mathbb{R}^{3} \times(0, \infty)\right)$, we have

$$
\begin{aligned}
& \frac{R}{\gamma-1} \int_{0}^{\infty} \int \rho^{\delta} \theta^{\delta}\left(\varphi_{t}+u^{\delta} \cdot \nabla \varphi\right) d x d t-\kappa \int_{0}^{\infty} \int \nabla \theta^{\delta} \cdot \nabla \varphi d x d t \\
& =-\int_{0}^{\infty} \int G^{\delta} \operatorname{div} u^{\delta} \varphi d x d t+R \int_{0}^{\infty} \int \operatorname{div} u^{\delta} \varphi d x d t \\
& \quad+2 \mu \int_{0}^{\infty} \int\left(u^{\delta} \cdot \nabla u^{\delta}-u^{\delta} \operatorname{div} u^{\delta}\right) \cdot \nabla \varphi d x d t-\frac{\mu}{2} \int_{0}^{\infty} \int\left|\omega^{\delta}\right|^{2} \varphi d x d t .
\end{aligned}
$$

Letting $\delta=\delta_{j}$ in (5.57) and taking appropriate limits, we thus deduce from (5.47), (5.46), (5.48), and (5.50) that

$$
\begin{aligned}
& \frac{R}{\gamma-1} \int_{0}^{\infty} \int \rho \theta\left(\varphi_{t}+u \cdot \nabla \varphi\right) d x d t-\kappa \int_{0}^{\infty} \int \nabla \theta \cdot \nabla \varphi d x d t \\
& =-\int_{0}^{\infty} \int G \operatorname{div} u \varphi d x d t+R \int_{0}^{\infty} \int \operatorname{div} u \varphi d x d t \\
& \quad+2 \mu \int_{0}^{\infty} \int(u \cdot \nabla u-u \operatorname{div} u) \cdot \nabla \varphi d x d t-\frac{\mu}{2} \int_{0}^{\infty} \int|\omega|^{2} \varphi d x d t \\
& =-\int_{0}^{\infty} \int(\lambda \operatorname{div} u-P) \operatorname{div} u \varphi d x d t-2 \mu \int_{0}^{\infty} \int|\mathfrak{D}(u)|^{2} \varphi d x d t \\
& \quad+2 \mu \int_{0}^{\infty} \int\left(\partial_{k} u^{i} \partial_{i}\left(u^{k} \varphi\right)-\operatorname{div}(u \varphi) \operatorname{div} u\right) d x d t \\
& =\int_{0}^{\infty} \int_{P \operatorname{div} u \varphi d x d t-\int_{0}^{\infty} \int\left(\lambda(\operatorname{div} u)^{2}+2 \mu|\mathfrak{D}(u)|^{2}\right) \varphi d x d t}
\end{aligned}
$$


where in the last equality, we have used the following simple fact that, for standard mollifier $j_{\nu}(x)$,

$$
\begin{aligned}
& \left|\int_{0}^{\infty} \int\left(\partial_{k} u^{i} \partial_{i}\left(u^{k} \varphi\right)-\operatorname{div}(u \varphi) \operatorname{div} u\right) d x d t\right| \\
& =\mid \int_{0}^{\infty} \int \partial_{k}\left(u^{i}-u^{i} * j_{\nu}\right) \partial_{i}\left(u^{k} \varphi\right) d x d t \\
& \quad+\int_{0}^{\infty} \int\left(\partial_{k}\left(u^{i} * j_{\nu}\right) \partial_{i}\left(u^{k} \varphi\right)-\operatorname{div}(u \varphi) \operatorname{div} u\right) d x d t \mid \\
& =\left|\int_{0}^{\infty} \int\left(\partial_{k}\left(u^{i}-u^{i} * j_{\nu}\right) \partial_{i}\left(u^{k} \varphi\right)+\operatorname{div}(u \varphi) \operatorname{div}\left(u * j_{\nu}-u\right)\right) d x d t\right| \\
& \leq C \int_{0}^{\infty} \int|\nabla(u \varphi)|\left|\nabla\left(u-u * j_{\nu}\right)\right| d x d t \rightarrow 0, \text { as } \nu \rightarrow 0,
\end{aligned}
$$

due to (1.29). We thus derive (1.31) directly from (5.58), (5.48), and (5.47).

Finally, to finish the proof of Theorem 1.2, it remains to prove (1.27). Since $(\rho, u)$ satisfies (1.18), for the standard mollifier $j_{\nu}(x)(\nu>0), \rho^{\nu} \triangleq \rho * j_{\nu}$ satisfies

$$
\left\{\begin{array}{l}
\rho_{t}^{\nu}+\operatorname{div}\left(u \rho^{\nu}\right)=r_{\nu}, \\
\rho^{\nu}(x, t=0)=\rho_{0} * j_{\nu},
\end{array}\right.
$$

where $r_{\nu}$ satisfies, for any $T>0$,

$$
\lim _{\nu \rightarrow 0^{+}} \int_{0}^{T}\left\|r_{\nu}\right\|_{L^{2}}^{2} d t=0,
$$

due to (3.9), (3.6), and [16, Lemma 2.3]. Multiplying (5.59) by $4\left(\rho^{\nu}-1\right)^{3}$, we obtain after integration by parts that, for $t \geq 1$,

$$
\begin{aligned}
& \left(\left\|\rho^{\nu}-1\right\|_{L^{4}}^{4}\right)^{\prime} \\
& =-4 \int\left(\rho^{\nu}-1\right)^{3} \operatorname{div} u d x-3 \int\left(\rho^{\nu}-1\right)^{4} \operatorname{div} u d x+4 \int r_{\nu}\left(\rho^{\nu}-1\right)^{3} d x \\
& \leq C\left\|\rho^{\nu}-1\right\|_{L^{4}}^{4}+C\|\nabla u\|_{L^{4}}^{4}+C\left\|r_{\nu}\right\|_{L^{2}},
\end{aligned}
$$

which implies that, for all $1 \leq N \leq s \leq N+1 \leq t \leq N+2$,

$$
\begin{aligned}
\left\|\rho^{\nu}(\cdot, t)-1\right\|_{L^{4}}^{4} \leq & \left\|\rho^{\nu}(\cdot, s)-1\right\|_{L^{4}}^{4}+C \int_{N}^{N+2}\left(\left\|\rho^{\nu}-1\right\|_{L^{4}}^{4}+\|\nabla u\|_{L^{4}}^{4}\right) d t \\
& +C \int_{N}^{N+2}\left\|r_{\nu}\right\|_{L^{2}} d t .
\end{aligned}
$$

Letting $\nu \rightarrow 0^{+}$in (5.62) together with (5.60) and (1.23) yields that

$$
\|\rho(\cdot, t)-1\|_{L^{4}}^{4} \leq\|\rho(\cdot, s)-1\|_{L^{4}}^{4}+C \int_{N}^{N+2}\left(\|\rho-1\|_{L^{4}}^{4}+\|\nabla u\|_{L^{4}}^{4}\right) d t .
$$

Integrating (5.63) with respect to $s$ over $[N, N+1]$ leads to

$$
\begin{aligned}
\sup _{t \in[N+1, N+2]}\|\rho(\cdot, t)-1\|_{L^{4}}^{4} & \leq C \int_{N}^{N+2}\left(\|\rho-1\|_{L^{4}}^{4}+\|\nabla u\|_{L^{4}}^{4}\right) d t \\
& \rightarrow 0, \text { as } N \rightarrow \infty
\end{aligned}
$$


due to (5.51). This together with (1.26) and (1.29) implies that, for all $p \in(2, \infty)$,

$$
\lim _{t \rightarrow \infty} \int|\rho-1|^{p} d x=0 .
$$

Finally, we will prove

$$
\lim _{t \rightarrow \infty}\left(\|u\|_{L^{4}}+\|\nabla \theta\|_{L^{2}}\right)=0,
$$

which combining with (5.65), (1.26), (1.28)-(1.30), and the Gagliardo-Nirenberg inequality thus gives (1.27). In fact, one deduces from (1.28)-(1.30) that

$$
\begin{aligned}
& \int_{1}^{\infty}\left(\|u\|_{L^{4}}^{4}+\|\nabla \theta\|_{L^{2}}^{2}\right) d t \\
& \leq C \int_{1}^{\infty}\|u\|_{L^{2}}\|\nabla u\|_{L^{2}}^{3} d t+\int_{1}^{\infty}\|\nabla \theta\|_{L^{2}}^{2} d t \\
& \leq C, \\
& \int_{1}^{\infty}\left|\frac{d}{d t}\left(\|u(\cdot, t)\|_{L^{4}}^{4}\right)\right| d t=\left.4 \int_{1}^{\infty}\left|\int\right| u\right|^{2} u \cdot u_{t} d x \mid d t \\
& \leq C \int_{1}^{\infty}\|u\|_{L^{\infty}}\|u\|_{L^{4}}^{2}\left\|u_{t}\right\|_{L^{2}} d t \\
& \leq C,
\end{aligned}
$$

and

$$
\begin{aligned}
\int_{1}^{\infty}\left|\frac{d}{d t}\left(\|\nabla \theta(\cdot, t)\|_{L^{2}}^{2}\right)\right| d t & =2 \int_{1}^{\infty}\left|\int \nabla \theta \cdot \nabla \theta_{t} d x\right| d t \\
& \leq C \int_{1}^{\infty}\|\nabla \theta\|_{L^{2}}\left\|\nabla \theta_{t}\right\|_{L^{2}} d t \\
& \leq C .
\end{aligned}
$$

Thus, we derive (5.66) easily from (5.67)-(5.69). The proof of Theorem 1.2 is finished.

\section{References}

[1] S. N. Antontsev, A. V. Kazhikhov, V. N. Monakhov, Boundary value problems in mechanics of nonhomogeneous fluids, North-Holland Publishing Co., Amsterdam, 1990.

[2] J. T. Beale, T. Kato, A. Majda, Remarks on the breakdown of smooth solutions for the 3-D Euler equations, Commun. Math. Phys. 94 (1984), 61-66.

[3] D. Bresch, B. Desjardins, On the existence of global weak solutions to the NavierStokes equations for viscous compressible and heat conducting fluids. J. Math. Pures Appl. (9) 87 (2007), 57-90.

[4] Y. Cho, B.J. Jin, Blow-up of viscous heat-conducting compressible flows, J. Math. Anal. Appl. 320 (2006), 819-826.

[5] Y. Cho, H. Kim, On classical solutions of the compressible Navier-Stokes equations with nonnegative initial densities. Manuscript Math. 120 (2006), 91-129.

[6] Y. Cho, H. Kim, Existence results for viscous polytropic fluids with vacuum. J. Differential Equations 228 (2006), 377-411. 
[7] E. Feireisl, Dynamics of Viscous Compressible Fluids, Oxford Science Publication, Oxford, 2004.

[8] E. Feireisl, On the motion of a viscous, compressible, and heat conducting fluid, Indiana Univ. Math. J. 53 (2004), 1707-1740.

[9] E. Feireisl, A. Novotny, H. Petzeltová, On the existence of globally defined weak solutions to the Navier-Stokes equations. J. Math. Fluid Mech. 3 (2001), 358-392.

[10] D. Hoff, Discontinuous solutions of the Navier-Stokes equations for multidimensional flows of heat-conducting fluids. Arch. Rational Mech. Anal. 139 (1997), 303-354.

[11] X. D. Huang, J. Li, Global classical and weak solutions to the three-dimensional full compressible Navier-Stokes system with vacuum on bounded domains. In preparation.

[12] X. D. Huang, J. Li, Z. P. Xin, Serrin type criterion for the three-dimensional compressible flows. Siam J. Math. Anal. in press.

[13] X. D. Huang, J. Li, Z. P. Xin, Global well-posedness of classical solutions with large oscillations and vacuum to the three-dimensional isentropic compressible NavierStokes equations, Comm. Pure Appl. Math. in press.

[14] A. V. Kazhikhov, Cauchy problem for viscous gas equations, Siberian Math. J. 23 (1982), 44-49.

[15] A. V. Kazhikhov, V. V. Shelukhin, Unique global solution with respect to time of initial-boundary value problems for one-dimensional equations of a viscous gas, $J$. Appl. Math. Mech. 41 (1977), 273-282.

[16] P. L. Lions, Mathematical topics in fluid mechanics. Vol. 1. Incompressible models, Oxford University Press, New York, 1996.

[17] P. L. Lions, Mathematical topics in fluid mechanics. Vol. 2. Compressible models, Oxford University Press, New York, 1998.

[18] A. Matsumura, T. Nishida, The initial value problem for the equations of motion of viscous and heat-conductive gases, J. Math. Kyoto Univ. 20 (1980), 67-104.

[19] J. Nash, Le problème de Cauchy pour les équations différentielles d'un fluide général,Bull. Soc. Math. France. 90 (1962), 487-497.

[20] L. Nirenberg, On elliptic partial differential equations, Ann. Scuola Norm. Sup. Pisa (3) 13 (1959), 115-162.

[21] O. Rozanova, Blow up of smooth solutions to the compressible Navier-Stokes equations with the data highly decreasing at infinity, J. Differ. Eqs. 245 (2008), $1762-1774$.

[22] J. Serrin, On the uniqueness of compressible fluid motion, Arch. Rational. Mech. Anal. 3 (1959), 271-288.

[23] Z. P. Xin, Blowup of smooth solutions to the compressible Navier-Stokes equation with compact density. Comm. Pure Appl. Math. 51 (1998), 229-240. 